\title{
Full-Duplex Wireless Communications: Challenges, Solutions and Future Research Directions
}

\author{
Zhongshan Zhang, Senior Member, IEEE, Keping Long, Senior Member, IEEE, \\ Athanasios V. Vasilakos, Senior Member, IEEE and Lajos Hanzo*, Fellow, IEEE
}

\begin{abstract}
The family of conventional half-duplex (HD) wireless systems relied on transmitting and receiving in different time-slots or frequency sub-bands. Hence the wireless research community aspires to conceive full-duplex (FD) operation for supporting concurrent transmission and reception in a single time/frequency channel, which would improve the attainable spectral efficiency by a factor of two. The main challenge encountered in implementing an FD wireless device is the large power difference between the self-interference (SI) imposed by the device's own transmissions and the signal of interest received from a remote source. In this survey, we present a comprehensive list of the potential FD techniques and highlight their pros and cons. We classify the SI cancellation techniques into three categories, namely passive suppression, analog cancellation and digital cancellation, with the advantages and disadvantages of each technique compared. Specifically, we analyze the main impairments (e.g. phase noise, power amplifier nonlinearity as well as in-phase and quadrature-phase (I/Q) imbalance, etc.) that degrading the SI cancellation. We then discuss the FD based Media Access Control (MAC)-layer protocol design for the sake of addressing some of the critical issues, such as the problem of hidden terminals, the resultant end-to-end delay and the high packet loss ratio (PLR) due to network congestion. After elaborating on a variety of physical/MAC-layer techniques, we discuss potential solutions conceived for meeting the challenges imposed by the aforementioned techniques. Furthermore, we also discuss a range of critical issues related to the im-
\end{abstract}

Zhongshan Zhang and Keping Long are with Beijing Engineering and Technology Center for Convergence Networks and Ubiquitous Services, University of Science and Technology Beijing (USTB), No. 30, Xueyuan Road, Haidian District, Beijing, China 100083 (e-mail: \{zhangzs,longkeping\}@ ustb.edu.cn).

Athanasios V. Vasilakos is with the Dept of Computer Science, Electrical and Space Engineering 97187, Lulea University of Technology, Lulea, Sweden (e-mail: th.vasilakos@gmail.com).

Lajos Hanzo is with the School of Electronics and Computer Science, University of Southampton, Southampton, SO17 1BJ, UK (email: 1h@ecs.soton.ac.uk).

This work was supported by the key project of the National Natural Science Foundation of China (No. 61431001), Program for New Century Excellent Talents in University (NECT-12-0774) and the Foundation of Beijing Engineering and Technology Research Center for Convergence Networks. The financial support of the EPSRC, UK under the poject EP/N004558/1 and of the ERC's Advanced Fellow Grant is gratefully acknowledged. plementation, performance enhancement and optimization of FD systems, including important topics such as hybrid FD/HD scheme, optimal relay selection and optimal power allocation, etc. Finally, a variety of new directions and open problems associated with FD technology are pointed out. Our hope is that this treatise will stimulate future research efforts in the emerging field of FD communications.

Index Terms-Full-Duplex, Self-Interference, Passive Suppression, Analog Cancellation, Digital Cancellation, Phase Noise, Power Amplifier Nonlinearity, Transmit I/Q Imbalance, Relay, Amplify-and-Forward, Decode-andForward, Optimal Power Allocation, Optimal Relay Selection, Cognitive Radio.

\section{INTRODUCTION}

$\mathbf{S}$ INCE communication networks are expected to deliver ever-increasing data rates, the spectral efficiency of the networks has to be further improved. Although some advanced techniques, such as MultiInput Multi-Output (MIMO) [1], [2] and Orthogonal Frequency Division Multiplexing (OFDM) [3] have been identified as promising solutions for beneficially increasing the network's spectral efficiency, the currently operational wireless communication systems are still unable to sufficiently satisfy the above-mentioned requirements, because today's systems usually employ devices that use either a time-division or frequency-division duplex for the signals' transmission and reception. Hence in practice only half-duplex (HD) operations have been employed, leading to an erosion of the resource utilization (i.e. the half-duplex-induced factor two capacity degradation cannot be avoided) [4], [5].

In order to compensate for the shortcomings of the HD systems, the promise of radical full-duplex (FD) operation [6], on the other hand, improves the achievable spectral efficiency of wireless communication systems by avoiding the utilization of two independent channels for bi-directional end-to-end transmission that is inherent in the conventional HD operations. Many of the current research achievements have already demonstrated the 
TABLE I

Performance Comparison among Existing Si Cancellation Techniques

\begin{tabular}{|c|c|c|c|c|c|c|c|}
\hline Algorithm & $\begin{array}{l}\text { Transmit } \\
\text { Power }\end{array}$ & $\begin{array}{l}\text { Center } \\
\text { Frequency }\end{array}$ & Bandwidth & $\begin{array}{l}\text { Antenna } \\
\text { Distances }\end{array}$ & $\begin{array}{l}\text { Antenna } \\
\text { Separations }\end{array}$ & $\begin{array}{l}\text { Cancellation } \\
\text { Capability }\end{array}$ & $\begin{array}{l}\text { Full-Duplex } \\
\text { Gain }\end{array}$ \\
\hline $\begin{array}{l}\text { Antenna } \\
\text { Cancellation [7] }\end{array}$ & $0 \mathrm{dBm}$ & $2.4 \mathrm{GHz}$ & $5 \mathrm{MHz}$ & $\overline{2 d+\lambda / 2}$ & & $60 \mathrm{~dB}$ & 1.84 \\
\hline AS [12] & $\begin{array}{l}-5 \mathrm{dBm} \\
\sim 15 \mathrm{dBm}\end{array}$ & $2.4 \mathrm{GHz}$ & $625 \mathrm{kHz}$ & $\frac{20 \mathrm{~cm}}{40 \mathrm{~cm}}$ & & $\frac{39 \mathrm{~dB}}{45 \mathrm{~dB}}$ & \\
\hline ASDC [12] & $\begin{array}{l}-5 \mathrm{dBm} \\
\sim 15 \mathrm{dBm}\end{array}$ & $2.4 \mathrm{GHz}$ & $625 \mathrm{kHz}$ & $\begin{array}{l}20 \mathrm{~cm} \\
40 \mathrm{~cm}\end{array}$ & & $\begin{array}{l}70 \mathrm{~dB} \\
76 \mathrm{~dB}\end{array}$ & \\
\hline ASAC [12] & $\begin{array}{l}-5 \mathrm{dBm} \\
\sim 15 \mathrm{dBm}\end{array}$ & $2.4 \mathrm{GHz}$ & $625 \mathrm{kHz}$ & $\begin{array}{l}20 \mathrm{~cm} \\
40 \mathrm{~cm}\end{array}$ & & $\begin{array}{l}72 \mathrm{~dB} \\
76 \mathrm{~dB}\end{array}$ & \\
\hline ASADC [12] & $\begin{array}{l}-5 \mathrm{dBm} \\
\sim 15 \mathrm{dBm}\end{array}$ & $2.4 \mathrm{GHz}$ & $625 \mathrm{kHz}$ & $\begin{array}{l}20 \mathrm{~cm} \\
40 \mathrm{~cm}\end{array}$ & & $\begin{array}{l}78 \mathrm{~dB} \\
80 \mathrm{~dB}\end{array}$ & \\
\hline $\begin{array}{l}\text { Directional } \\
\text { Diversity [13] }\end{array}$ & $12 \mathrm{dBm}$ & $2.4 \mathrm{GHz}$ & $20 \mathrm{MHz}$ & $\begin{array}{l}10 \mathrm{~m} \\
15 \mathrm{~m}\end{array}$ & $\begin{array}{l}\geq 45^{\circ} \\
\geq 90^{\circ}\end{array}$ & & $\begin{array}{l}1.6 \sim 1.9 \\
\geq 1.4\end{array}$ \\
\hline $\begin{array}{l}\text { QCNTA [14] } \\
\text { SCSI [15] }\end{array}$ & $\frac{-3 \mathrm{dBm}}{6 \mathrm{dBm}}$ & $\begin{array}{l}530 \mathrm{MHz} \\
2.4 \mathrm{GHz}\end{array}$ & $\begin{array}{l}20 \mathrm{MHz} \\
10 \mathrm{MHz}\end{array}$ & & & $\begin{array}{l}55 \mathrm{~dB} \\
58 \sim 8 \mathrm{~dB}\end{array}$ & $1 \sim 2$ \\
\hline $\begin{array}{l}\text { ScST [15] } \\
\text { TDTB [16] }\end{array}$ & $\begin{array}{l}6 \mathrm{dBm} \\
17 \mathrm{dBm}\end{array}$ & $\frac{2.4 \mathrm{GHz}}{2.4 \mathrm{GHz}}$ & $\begin{array}{l}10 \mathrm{MHz} \\
30 \mathrm{MHz}\end{array}$ & $\leq 33 \mathrm{~cm}$ & & $\frac{58 \sim 81 \mathrm{~dB}}{50 \mathrm{~dB}}$ & $\geq 1.7$ \\
\hline ZigZag [17] & & $2.4 \mathrm{GHz}$ & & & & & 1.25 \\
\hline $\begin{array}{l}\text { Balun [10] } \\
\text { Circulator } \mid 110\end{array}$ & $20 \mathrm{dBm}$ & $\begin{array}{l}2.4 \mathrm{GHz} \\
2.4 \mathrm{GHz}\end{array}$ & 10-40MHz & $20 \mathrm{~cm}$ & & $113 \mathrm{~dB}$ & 1.45 \\
\hline $\begin{array}{l}\text { Circulator [11] } \\
\text { SDR Platform [18] }\end{array}$ & $20 \mathrm{dBm}$ & $\begin{array}{l}2.4 \mathrm{GHz} \\
2.52 \mathrm{GHz}\end{array}$ & $\begin{array}{l}20-80 \mathrm{MHZ} \\
20 \mathrm{MHz}\end{array}$ & & & $103 \mathrm{~dB}$ & $\begin{array}{l}1.81 \\
1.9\end{array}$ \\
\hline
\end{tabular}

feasibility of FD communication in practical systems [7], [8]. For example, some rudimentary FD devices, which are capable of transmitting and receiving all the time in the entire bandwidth [9], have been proposed. A pair of HD nodes can thus exchange their own data via this FD device. Furthermore, with the rapid progress of FD techniques, the commercial FD platforms have been widely developed by several research institutes (e.g. please refer to [7], [10], [11] for details). Most of them developed Wireless Fidelity (WiFi)-like platforms operating at $2.4 \mathrm{GHz}$ with bandwidth with bandwidth ranging from $20 \mathrm{MHz}$ to $80 \mathrm{MHz}$. Some well-designed platforms (e.g. [11]) are even capable of providing the FD capability that are consistent across a variety of bandwidths, constellations, transmit powers, etc, whilst almost without distorting the received signal. Additionally, several Media Access Control (MAC)-layer protocols specifically designed for FD systems have also been proposed for effectively mitigating the problems that are commonly observed in conventional HD systems (e.g. hidden terminal collisions) and fully investigating the FD benefits, thus delivering close to the theoretical doubling of throughput expected from FD mode. In Table I, the FD gains of the existing platforms are compared.

\section{A. Motivation}

Despite of above-mentioned benefits offered by FD techniques, as a downside, none of the existed FD techniques can experimentally attain the theoretical doubling gain in terms of the capacity/throughput, because the practical platforms always suffer from an Signal-toInterference-and-Noise Radio (SINR) loss due to the impact of self-interference (SI), which is caused by the large power difference between the interference signal imposed by a device's own wireless transmissions and the received signal of interest arriving from a remote transmit antenna (TA) [19], [20]. In fact, heavy SI may even result in a reduced capacity for the FD systems that falls below that of the HD systems. Furthermore, SI may render the communication systems unstable and might even lead to oscillations within the transceivers [21]. Consensus reached by both academia [22] and industry [23], [24] showed that SI suppression/cancellation would play the most critical role in implementing radical FD communication systems.

Recently, techniques of SI suppression/cancellation have been widely studied [7], [13], [25]-[27]. For instance, Radio Frequency (RF) interference cancellation is employed in [25] to achieve FD operation. Furthermore, a plethora of studies has been carried out to perform SI suppression and cancellation, including techniques such as passive suppression [19] or analog and digital cancellation [7], [13] (please refer to Section III for details). The main progress associated with SI suppression/cancellation in FD communication is reflected in the following aspects.

1) Before performing analog- and/or digital-domain SI cancellation, diverse techniques capable of achieving a high isolation between the TA and receive antenna (RA) can be employed for suppressing the SI strength [28]. For instance, the signal transmitted on the air can be attenuated by the path-loss effect (proportional to the TARA distance), enabling a technique referred to as passive suppression [7] to be employed for reducing the SI prior to it impinging upon the RA.

2) Analog cancellation is capable of preventing the high-power SI inflicted by the analog-to-digital 
converter (ADC), which would desensitize the automatic gain control (AGC) owing to signal leakages. Either training sequence-based methods [12] or adaptive interference cancellation [7] may be used for performing analog cancellation.

3) In light of the fact that even after performing analog cancellation the residual SI encountered in practical systems remains the rate-limiting bottleneck, additional digital cancellation is required for mitigating the interference effects in the baseband [12]. Since both the linear and nonlinear residual SI components should be subtracted in the digital domain [11], the FD receiver is required to estimate both the delay and phase shift between the transmitted and the received signals relying on SI channel estimates.

4) Note that no stand-alone analog or digital technique is capable of achieving a high enough cancellation capability to satisfy the decoding requirement. It was reported in [7], [12] that the interference rejection ratio of analog-only methods only ranges from 20 to $45 \mathrm{~dB}$, making the residual SI may still be several-dB above the thermal noise floor even with the help of passive suppression [4]. Therefore, a combination of analog and digital cancellations may be promising to offer a sufficiently high SI cancellation capability.

However, the hardware imperfections encountered in practical FD realizations, such as nonlinear distortions, non-ideal frequency response of circuits, the phase noise, etc., may impose significant performance limitations on the SI cancellation, thus resulting in a high residual SI [11], [29]. It was shown in [29] that the phase noise may gravely limit the combined analog and digital SI cancellation. If the residual SI is uncorrelated with the SI signal, the phase noise will dominate the residual SI (i.e. after performing analog cancellation) and prevent the concatenated digital canceller from further cancelling the residual SI. Apart from the aforementioned issues, the conception of FD MAC-layer protocols requires substantial further research for exploiting the FD benefits potentially offered by the physical-layer techniques [15]. Some of the most challenging problems in wireless networks, such as the presence of hidden terminals, loss of throughput due to congestion and large endto-end delays, etc. have to be mitigated by carefully designed FD MAC-layer protocols [7]. Furthermore, the existing studies indicated that FD schemes may not always outperform their HD counterparts, hence hybrid schemes switching between the HD mode and FD mode have to be developed for adaptively exploiting the radio resources, while at the same time maximizing both the instantaneous and the average spectral efficiency [22].

To sum up, one of the fundamental motivations of this paper is to survey and analyze the critical techniques that enable the wireless devices to operate in FD mode. Since the FD mode allows wireless devices to concurrently transmit and receive in a single time/frequency-slot, it significantly improves both the attainable spectral efficiency and the resource utilization. We will survey and compare different FD techniques and introduce various SI suppression/cancellation schemes. Some existing SI cancellation techniques such as passive suppression, as well as both analog and digital cancellations will be discussed, with their advantages, drawbacks and open design challenges being analyzed. Specifically, the hardware limitations encountered in practical FD system design, including the phase noise, the frequency nonideal response, the power amplifier nonlinearity, as well as the transmit in-phase and quadrature-phase (I/Q) imbalance have to be investigated. Furthermore, some critical issues related to the MAC-layer protocols are also studied. Finally, advanced practical implementations and commercial realizations of hybrid FD/HD scheme, optimal relay selection, and optimal power allocation are discussed, followed by a variety of new directions and open problems.

\section{B. Main Contributions}

In this paper, we review the state of the art in FD wireless communication system design and investigate the critical techniques such as SI suppression/cancellation and MAC-layer protocols, while highlighting the distortions imposed by hardware imperfections encountered in practical systems. The main contributions of this survey include

1) Outlining the potential benefits offered by FD techniques.

2) Surveying the critical issues related to FD transmissions from a physical-layer perspective relying on SI suppression/cancellation, whilst giving cognizance to the MAC-layer protocols.

3) Investigating the main hardware imperfections, such as the phase noise, non-flat frequency response of circuits, power amplifier nonlinearity, and transmit I/Q imbalance, etc., which may impose limitations on the attainable SI cancellation capability.

4) Discussing the advantages, drawbacks, as well as design challenges of practical FD systems, whilst identifying their new directions and applications. 
The remainder of this paper is organized as follows. The main benefits brought about by employing FD techniques will be listed in Section II. Then a range of SI cancellation techniques, including passive suppression, analog and digital cancellations, will be introduced in Section III. Specifically, the impacts of hardware limitations (e.g. phase noise, power amplifier nonlinearity and transmit I/Q imbalance) on SI cancellation are elaborated in Section III-E. Typical FD MAC-layer protocols, such as the Busytone-aided MAC Protocol [10] and the FD-MAC technique [15] are then discussed in Section IV, followed by a range of critical issues related to the associated practical implementation and commercial realizations in Section V. Finally, our conclusions as well as future research directions are provided in Section VI.

\section{BENEFITS OF EMPLOYING FULL-DUPLEX OPERATIONS}

Recent advances in FD communications have increased both the attainable throughput and diversity orders of systems communicating over wireless channels. The main driving force behind the advances in FD communications is the promise of nearly doubled channel capacity compared to the conventional HD communications, while meeting a range of contradicting design challenges. For example, it is possible to both enhance the SI cancellation and simultaneously to reduce the Bit Error Ratio (BER), provided that an increased complexity may be tolerated to facilitate more sophisticated signal processing. Similarly, the packet loss ratio (PLR) may also be further reduced, if a larger buffer size may be provided by the FD devices. In a nutshell, the FD mode exhibits advantages over the HD mode in terms of either having an increased throughput or reduced outage probability (OP) [30], [31] albeit this is achieved at the cost of increased complexity (i.e. for performing SI cancellation).

Nonetheless, at the time of writing HD rather than FD regimes may be preferred owing to the following reasons:

- Although in theory the FD mode may be capable of doubling the capacity of the HD mode, the latter becomes a more attractive choice in the presence of excessive SI, which prevents high-integrity communication in the FD mode [30].

- HD modes may be more attractive from the perspective of low-complexity practical implementation [32].

Again, an FD scheme may not always outperform its HD counterpart in all scenarios, hence a hybrid HD/FD scheme may be proposed to gain an advantage over either of the individual schemes. In a word, the attainable performance benefits depend not only on the SIcancellation capability, but also on a range of practical implementation issues, although the former acts as the dominant factor.

Recently, a range of theoretical and practical aspects of FD communications have been investigated [22], [33] by quantifying the performance of FD modes. In this section, we investigate the advantages/disadvantages of FD technology, with a range of further plausible tradeoffs identified. A comparison of the HD modes and FD modes will be carried out from a capacity, OP, and BER perspective. It is worth noting that the conclusions drawn in this section are applicable not only to cooperative relays, although representative performance comparisons between HD and FD modes may be readily carried out in cooperative relaying scenarios. For example, the FD benefits of relaying systems quantified in terms of capacity/throughput improvements over the HD mode can also be readily gleaned in device-to-device (D2D)like scenarios [10], [11], where a pair of FD nodes simultaneously send packets to each other. For the reader's convenience, we have summarized the major contributions on the subject of performance comparisons in Table II.

\section{A. Capacity Comparison of HD and FD Modes}

The achievable capacity/throughput in FD systems has been widely analyzed in the existing studies (e.g., [7], [10], [11], [15]). In theory, FD techniques may double the capacity of the HD techniques due to the potential capability of simultaneously transmitting and receiving using the same channel in the former. Although SI may substantially degrade the integrity of the FD mode, in some scenarios this might be tolerable [36]. In the following parts, capacity of FD systems in either Amplifyand-Forward (AF) or Decode-and-Forward (DF) mode will be investigated.

1) AF Mode: In the infrastructure-based AF relaying mode relying on flat-fading wireless links, the end-to-end channel capacities of the FD/HD modes were derived in [30], leading to the following conclusions:

- If the power of SI can be reduced below the noise level, the FD mode always outperforms the HD mode, regardless of the channel signal-to-noise ratio (SNR).

- The HD mode outperforms the FD mode only in the scenario when the SNR of the source $\rightarrow$ relay link is rather low and the relay's input SINR is dominated by the SI. 
TABLE II

Major Performance Comparison Between HD modes AND FD modes

\begin{tabular}{|c|c|c|c|c|}
\hline Year & Author(s) & Contribution & Complexity & Assumption \\
\hline 2005 & $\begin{array}{l}\text { Kramer et } \\
\text { al. [34] }\end{array}$ & $\begin{array}{l}\text { Proves that transmit power optimization } \\
\text { is capable of alleviating the effect of } \\
\text { SI and exhibiting the advantages of FD } \\
\text { protocols. }\end{array}$ & Low & $\begin{array}{l}\text { The DF relays can successfully decode } \\
\text { the message. }\end{array}$ \\
\hline \multirow[t]{2}{*}{2008} & $\begin{array}{l}\text { Schoenen et } \\
\text { al. [32] }\end{array}$ & $\begin{array}{l}\text { Shows that the HD mode with sophis- } \\
\text { ticated protocol design may also be at- } \\
\text { tractive from the perspective of practical } \\
\text { implementation. }\end{array}$ & Medium & $\begin{array}{l}\text { Resources are partitioned for the multi- } \\
\text { hop links by quasi-static resource parti- } \\
\text { tioning unit. }\end{array}$ \\
\hline & $\begin{array}{l}\text { Nikjah et al. } \\
\text { [35] }\end{array}$ & $\begin{array}{l}\text { Describes rateless coded HD and FDDF } \\
\text { protocols utilizing opportunistic relaying, } \\
\text { and examines and compares their achiev- }\end{array}$ & Low & $\begin{array}{l}\text { A peak power constraint and an average } \\
\text { power constraint are assumed. }\end{array}$ \\
\hline \multirow[t]{3}{*}{2009} & $\begin{array}{l}\text { Riihonen et } \\
\text { al. [36] }\end{array}$ & $\begin{array}{l}\text { Evaluates the break-even SI level and } \\
\text { shows when the FD mode can offer ca- } \\
\text { pacity improvement over its HD counter- } \\
\text { part. }\end{array}$ & Low & $\begin{array}{l}\text { Loop interference in FD relay can be } \\
\text { made below the break-even level. }\end{array}$ \\
\hline & $\begin{array}{l}\text { Riihonen et } \\
\text { al. [30] }\end{array}$ & $\begin{array}{l}\text { Derives the end-to-end channel capaci- } \\
\text { ties of the HD and FD modes in the } \\
\text { infrastructure-based AF protocols. }\end{array}$ & Low & $\begin{array}{l}\text { The loop interference and the interference } \\
\text { due to frequency reuse are at the same } \\
\text { level as the receiver noise power. }\end{array}$ \\
\hline & $\begin{array}{l}\text { Skraparlis et } \\
\text { al. [37] }\end{array}$ & $\begin{array}{l}\text { Determines the outage probability for } \\
\text { both the HD and FD modes in the cor- } \\
\text { related lognormal channels. }\end{array}$ & Low & $\begin{array}{l}\text { Correlated lognormal channels are con- } \\
\text { sidered. }\end{array}$ \\
\hline \multirow[t]{2}{*}{2010} & $\begin{array}{l}\text { Riihonen et } \\
\text { al. [38] }\end{array}$ & $\begin{array}{l}\text { Demonstrates that the FD modes can } \\
\text { achieve almost doubled data rate in com- } \\
\text { parison to their HD counterpart even with } \\
\text { residual SI. }\end{array}$ & Low & $\begin{array}{l}\text { FD relays can improve the end-to-end ca- } \\
\text { pacity by adjusting their transmit power. }\end{array}$ \\
\hline & $\begin{array}{l}\text { Kwon et al. } \\
\text { [39] }\end{array}$ & $\begin{array}{l}\text { Performs the comparison between the HD } \\
\text { modes and FD modes to show the optimal } \\
\text { duplex mode for DF relay in terms of } \\
\text { outage probability. }\end{array}$ & Medium & Rayleigh fading channels are assumed. \\
\hline \multirow[t]{2}{*}{2011} & $\begin{array}{l}\text { Sahai et al. } \\
\text { [15] }\end{array}$ & $\begin{array}{l}\text { DHardware implementations show that } \\
\text { over } 70 \% \text { throughput gains from using } \\
\text { FD mode over HD mode in realistically } \\
\text { used cases. }\end{array}$ & High & Optimal antenna placement is feasible. \\
\hline & $\begin{array}{l}\text { Riihonen et } \\
\text { al. [40] }\end{array}$ & $\begin{array}{l}\text { Proposes optimal power allocation for } \\
\text { spatial streams in FD DF MIMO relay- } \\
\text { ing networks to render power saving and } \\
\text { improve the system performance. Rate- } \\
\text { interference tradeoff between the HD } \\
\text { modes and FD modes is also studied. }\end{array}$ & High & $\begin{array}{l}\text { Sl can be directed to the least harmful } \\
\text { dimensions by using multi-antenna tech- } \\
\text { niques. }\end{array}$ \\
\hline \multirow[t]{2}{*}{2012} & $\begin{array}{l}\text { Hiep et al. } \\
\text { [41] }\end{array}$ & $\begin{array}{l}\text { Evaluates the channel capacity of a multi- } \\
\text { hop HD/FD mode relay system. }\end{array}$ & Low & $\begin{array}{l}\text { Flat Rayleigh fading channels are as- } \\
\text { sumed. }\end{array}$ \\
\hline & $\begin{array}{l}\text { Alves et al. } \\
\text { [42] }\end{array}$ & $\begin{array}{l}\text { Compares the performance of FD relay- } \\
\text { ing with the incremental redundancy (IR)- } \\
\text { assisted HD relaying. }\end{array}$ & Low & Flat fading channel is assumed. \\
\hline \multirow[t]{3}{*}{2013} & $\begin{array}{l}\text { Zheng et al. } \\
\text { [43] }\end{array}$ & $\begin{array}{l}\text { Derives the closed-form expressions of } \\
\text { the FD block Markov relay by con- } \\
\text { sidering independent non-identically dis- } \\
\text { tributed Nakagami-m fading and SI. }\end{array}$ & Medium & Nakagami- $m$ fading is assumed. \\
\hline & $\begin{array}{l}\text { Khafagy et } \\
\text { al. }[44]\end{array}$ & $\begin{array}{l}\text { Evaluates a selective DF protocol in FD } \\
\text { relaying system. }\end{array}$ & Medium & $\begin{array}{l}\text { The relay has knowledge of channel } \\
\text { statistics. }\end{array}$ \\
\hline & $\begin{array}{l}\text { Zlatanov } \\
\text { al. [45] }\end{array}$ & $\begin{array}{l}\text { Studies buffer-aided HD mode relaying } \\
\text { that outperforms ideal FD mode. }\end{array}$ & Medium & $\begin{array}{l}\text { Ideal FD relaying is considered, where SI } \\
\text { is assumed to be completely avoided. }\end{array}$ \\
\hline
\end{tabular}

2) DF Mode: The FD capacity of the DF mode has been widely studied for both Single-Input SingleOutput (SISO) [22] and MIMO systems [46]. In practical scenarios, the performance of the relaying systems has been shown to be significantly influenced by the buffer-fullness of the relays [47], leading to detailed investigations of the tradeoffs between the HD and FD modes in order to determine which mode shows a better upper-layer performance. In [31], the joint effects of finite-length queues and adaptive modulation on the family of DF-mode systems have been evaluated under uncorrelated Rayleigh fading conditions. A finite-state
Markov chain based model of both the HD and FD modes was proposed in [31] for DF systems relying on adaptive modulation. Similar solutions were also advocated in [48], whose contributions lead to the following conclusions:

- Both the PLR and the delay are found to be more severely degraded for the FD mode than for the HD mode, because the SI limits the number of packets departing from the queue.

- The number of packets discarded (either due to limited buffer size or owing to its excessive delay) often becomes higher in the FD mode than in the 
HD mode in the presence of SI. However, the FD mode may become superior to the HD mode if the size of the queue's buffer is sufficiently high. Unless the number of packets arriving from the upper layer becomes so high that it hinders the reliable packet transmission, increasing the buffer size generally benefits the FD mode more drastically than HD mode.

\section{B. Comparison of the HD and FD Modes in Terms of Outage Probability}

Apart from capacity, the OP of wireless links also constitutes one of the most important reliability metrics in a fading channel. In order to optimize the FD systems in terms of OP performance, the fundamental tradeoff between the attainable resource efficiency and SI tolerance must be carefully investigated. In the following parts, the performance comparison between the FD and HD modes in terms of OP performance will be carried out.

1) $O P$ of the AF Relaying Mode: In [49], the outage performance of an FD based wireless AF relay link is studied in the presence of realistic non-ideal feedback information. A new relay protocol is proposed for cophasing the direct and relaying paths to enhance both the end-to-end SNR as well as the outage capacity. It has been shown that the FD mode is capable of offering performance improvements over both classic direct transmission and HD relaying, even if the feedback channel is of relatively low quality due to using a limited number of feedback bits. Furthermore, the following conclusions may be inferred:

- In the presence of an adequate direct source $\rightarrow$ destination link but in the absence of residual SI, FD AF relaying is expected to outperform HD AF relaying, even if the SNR is low. By contrast, as the SNR increases, the employment of HD relaying becomes preferable from the perspective of the attainable OP, because the FD relaying will suffer from distortions either due to the increased residual SI or due to its noise amplification, which in fact degrades a strong direct link.

- Mitigating the residual SI by using classic minimum mean square error (MMSE) criterion eased decision feedback equalization at the destination is indeed capable of achieving better outage performance than HD relaying [50].

2) $O P$ of the DF Relaying Mode: In a multihop FD relaying system, where multiple DF mode relays are cascaded, the ability of the relays to isolate transmission from reception may be quantified by defining a new parameter referred to as the Path-Loss-to-Interference Ratio (PLIR), which represents the ratio of the received desired signal power to the received interference power, when the transmit power is the same for the useful and interfering signals [51]. For a given PLIR, the optimal number of FD relays should be determined by minimizing the OP of a multihop network, whilst relying on an appropriately designed protocol, such as selective DF [44]. The specific design-dilemma in this context is whether to use a lower number of less-reliable long hops, or a higher number of more reliable hops to minimize the OP.

Considering the DF relaying, the conditions to be satisfied for achieving superiority of FD mode over HD mode in terms of the OP can be summarized for a simple three-node cooperative network as follows:

- FD relaying is superior to conventional HD mode in terms of the OP when the Signal-to-Interference Ratio (SIR) is low (i.e. corresponding to a low SI strength) for transmission over Nakagami-m wireless channels [52]. As the SIR increases, FD mode tends to exhibit a lower OP than HD mode.

- When the SNR is low, the OP decreases upon increasing the number of relays in a multihop network, while this trend becomes reversed, as the SNR increases owing to the increased interference among the relays.

- For sufficiently high values of PLIR, the FD mode has been shown to outperform the HD mode in terms of the OP in a multihop relaying system.

\section{Comparison of HD modes and FD Modes in Terms of $B E R$}

The BER performance of FD systems has been lavishly documented. For instance, in [53], the BER of a MIMO-aided FD system has been evaluated with the assistance of beamforming for the sake of improving the effective SNR. Note that beamforming has been shown to be especially beneficial for AF relaying, which is prone to the performance-limitation imposed by the accumulated interference/noise [53].

1) BER of the AF Relaying Mode: In [54], the performance comparison of the AF relaying aided HD mode and FD mode was carried out in terms of the achievable BER, with the assistance of multiple antennas at each node. The source $\rightarrow$ destination beamforming vectors are jointly optimized in [54] based on the MMSE objective function subject to the transmit power constraints of both the source and the relays. Furthermore, a pre-nulling 
algorithm $^{1}$ is employed by the FD relays to facilitate SI suppression, provided that perfect channel state information (CSI) is available at each node. Naturally, the provision of accurate CSI for all nodes remains an open challenge at the time of writing. It has been demonstrated in [54] that the FD mode is capable of outperforming the HD mode in terms of its BER, when the SNR of the relay $\rightarrow$ destination link is lower than $5 \mathrm{~dB}$ due to the fact that the noise effect of the latter is twice as high as that of the former, but the situation is reversed, if the SNR of the relay $\rightarrow$ destination link becomes higher than $15 \mathrm{~dB}$.

2) BER of the DF Relaying Mode: In [33], the BER analysis of FD cooperative system employing a single DF relay is carried out in conjunction with binary phase shift keying (BPSK). Without loss of generality, the SI channel is assumed to suffer from Rayleigh or Nakagami-m fading. In contrast to the results obtained for AF relaying (e.g. in [54]), the closed-form BER expression derived for DF relaying demonstrates an inferior performance of the FD mode compared to that of the HD mode even for a low level of residual SI at both the relay and destination [33].

\section{Advantages/Disadvantages of the FD Mode}

Based on the aforementioned comparisons, the FD mode has shown several attractive advantages, but also exposed weaknesses in contrast to the HD mode. For example, since an FD node has to process twice as many packets as a HD node due to its simultaneous transmission and reception, both the PLR as well as the delay may become more severe for FD mode than for HD mode. Increasing their buffer's queue-length generally benefits FD mode more than HD mode. Nevertheless, striking the most appropriate buffer-size versus PLR tradeoff constitutes promising study-item. Both advantages and disadvantages of FD techniques are detailed below.

\section{1) Advantages of the Full-Duplex Mode:}

- Throughput Gain: As compared to the HD mode, the FD mode nearly doubles the throughput of a single-hop wireless link in the physical-layer.

- Collision Avoidance: In the traditional Carrier Sense Multiple Access with Collision Avoidance (CSMA/CA) protocol, each HD node is required to check the channel's quality before using it. The FD mode, however, only requires the first node that initiates transmissions to sense the channel, which is

\footnotetext{
${ }^{1}$ In MIMO-aided FD systems, the pre-nulling approach performs transmit pre-processing for the sake of minimizing SI imposed on the relay's TAs [55]. Pre-nulling algorithms will be specifically detailed in Section III-D.
}

necessary for avoiding collisions at those FD nodes that do not perform carrier sensing.

- Solving the Hidden Terminal Problem: The problem of hidden terminals can be solved using FD techniques. Let us consider a scenario of multiple nodes having data in their buffer for direct transmission to and reception from a common access point (AP). If a node starts transmitting its data to the AP and the AP simultaneously starts transmitting data back to this node, the other nodes will hear the transmissions from the AP and delay their transmissions to avoid collisions. Even if the AP has no data to send back to the first node, it still repeats an "ACK" for that node so as to prevent the other nodes from transmitting.

- Reducing Congestion with the Aid of MAC Scheduling: The potential throughput loss imposed by congestion can be circumvented by enabling FD operation in congested nodes. For instance, in a general star topology associated with $(2 n+1)$ nodes, nodes 1 to $n$ may attempt to transmit their data to nodes $(n+1)$ to $2 n$ respectively via node 0 . Then the aggregate network throughput becomes as low as $1 / n$ even if conventional HD MAC scheduling is performed. With the aid of FD operation, on the other hand, node 0 is capable of both transmitting and receiving simultaneously, hence the aggregate network throughput might approach the single-link capacity, whilst simultaneously benefitting from the spatial diversity gain.

- Reducing the End-to-End Delay: An FD node is capable of commencing the forwarding of a hitherto only partially received packet so as to significantly reduce the end-to-end delay of packet delivery through a multihop network, as compared to the conventional store-and-forward technique employed in HD mode, which would make the end-to-end delay a linearly increasing function of the number of hops.

- Enhancing the Primary User's Detection Quality in Cognitive Radio (CR) Environment: The reliable detection of the primary user is not an easy task to perform in CR environments [56]. This would, however, become an even more challenging operation, if the primary receivers were to operate only in a HD mode. As a benefit, the FD mode enables the secondary user to scan for any primary users, while it is actively occupying the spectrum. The primary receivers may transmit at the same time, so as to ease the secondary users' scanning and detection operation. 
2) Disadvantages of the Full-Duplex Mode:

- Performance Constrained by SI: In an FD device, the RA's input signal of interest is usually several orders of magnitude lower in power than the received SI signal imposed by the device's TA output. Hence, the interference imposed by the TA upon the RA will consequently drown out the weak input signal and degrade the FD gains.

- Degraded Link Reliability: The FD mode suffers from a reduced link reliability, regardless of the SNR. As indicated in [7], a state-of-the-art off-theshelf radio is capable of achieving $88 \%$ of the link reliability ${ }^{2}$ compared to its HD mode counterpart. Furthermore, without invoking digital interference cancellation, an even lower reliability of say $67 \%$ may be attainable for the FD mode.

- Suffers From Higher PLR: As compared to the HD devices, an FD node has to process twice the number of packets due to its simultaneous transmission and reception, thus leading to a higher PLR than the HD mode.

- A Higher Buffer Size Requirement: To reduce the PLR of the FD mode, a sufficiently large buffer is required for enabling the packets to be forwarded (that would otherwise have been discarded due to queue overflow). Since the effects of packet-loss level are more severe in the FD mode, a larger buffer size is required than for the HD mode.

A rudimentary performance comparison between the HD and FD modes is given in Table III. In practical implementations, the decision as to whether adopting the FD mode or the HD mode depends on several factors, such as the system throughput required, the SI cancellation capability, and the affordable hardware/software complexity, etc. Among the aforementioned factors, the SI signal significantly constrains the advantages of the FD techniques and would be the key limiting factor in developing FD systems. We will touch upon the inner core of this stylized illustration in the following two sections.

\section{SELF-INTERFERENCE CANCELLATION}

The goal of FD radio is to simultaneously transmit and receive within the same frequency band, in which case an FD node receives not only the signal of interest, but also the signal it is transmitting, which constitutes the SI imposed upon the RAs. Since the strength of the SI signal observed in FD devices may be 50-100 dB higher

\footnotetext{
${ }^{2}$ Link reliability may be described as the specific fraction of time during which a link between two adjacent nodes remains connected [57].
}

than that of the signal of interest, the strong SI signal will govern the gain control settings of the AGC, which scales the input signal prior to digitization to the normalized range of $[-1,1]$. If the SI power is high, it constrains the weak signal of interest to occupy a range much smaller than $[-1,1]$, hence invoking a high quantization noise on the signal of interest as well as a significantly eroding the SINR in the digital baseband [29].

To resolve the above-mentioned problem as well as to exploit the potential FD gains, we have to be capable of sufficiently reducing the SI strength before decoding the signal of interest [30]. For example, in a scenario relying on a FD radio having a transmit power of $0 \mathrm{dBm}$ and a noise floor of approximately $-90 \mathrm{dBm}$, the RAs have to be capable of reducing the SI by nearly $95 \mathrm{~dB}$ so as to ensure that the FD node's own transmissions do not unduly contaminate its reception [10]. As indicated in [20], the goal of SI cancellation is to predict and model the distortions in order to compensate for them at the RAs. However, SI cancellation is by no means a simple linear operation, because the conventional assumption that "the radio signal preserves its original baseband representation except for power scaling and frequency shifting" turns out to be partially incorrect [11]. To elaborate, in practical systems, the FD radios may distort the transmitted signal's digital baseband representation. Explicitly, both linear distortions (induced by signal attenuations and reflections from the environment, etc), as well as non-linear distortions (induced by circuit power leakage, non-flat hardware frequency response, higher-order signal harmonics, etc), the noise ${ }^{3}$ imposed by the imperfect transmit power amplifiers and phase noise 4 generated by local oscillators [11] are imposed. For example, in a typical WiFi radio using $80 \mathrm{MHz}$ bandwidth and a receiver noise floor of $-90 \mathrm{dBm}$ as well as the transmit power of $20 \mathrm{dBm}$, the SI signal comprises the following typical components [11]:

- The linear (main) component of $20 \mathrm{dBm}$ strength, corresponding to $110 \mathrm{~dB}$ above the noise floor;

- The non-linear component of $-10 \mathrm{dBm}$ strength, corresponding to $80 \mathrm{~dB}$ above the noise floor;

- The transmitter noise of $-40 \mathrm{dBm}$ strength, corresponding to $50 \mathrm{~dB}$ above the noise floor,

as graphically illustrated in Fig. 1.

In order to suppress the SI power to a level below the noise floor, the above-mentioned distortions must be adequately mitigated, whilst simultaneously considering the impact both of random transmitter noise and that of

\footnotetext{
${ }^{3}$ It was experimentally observed to be around the level of $-50 \mathrm{dBm}$, i.e. $40 \mathrm{~dB}$ higher than the receiver noise floor level of $-90 \mathrm{dBm}$ [58].

${ }^{4}$ It is typically of the order of $-40 \mathrm{dBm}[11]$.
} 
TABLE III

Performance Comparison Between HalF- AND Full-Duplex Schemes

\begin{tabular}{l||l|l}
\hline Technical Content & Half-Duplex & Full-Duplex \\
\hline \hline Throughput Gain & Lower & Higher (in theory 2 $\times$ that of HD mode) \\
\hline Self-Interference & Avoided & Cannot be avoided \\
\hline Hidden Terminal Collision & Suffered & Mitigated \\
\hline Congestion & Higher & Lower due to FD MAC scheduling \\
\hline End-to-end Delay & Higher & Lower \\
\hline Queue Size Requirement & Smaller & Larger \\
\hline PLR & Lower & Higher \\
\hline Link Reliability & Higher & Lower \\
\hline Primary User Detection in CR & Challenging & Improved \\
\hline
\end{tabular}

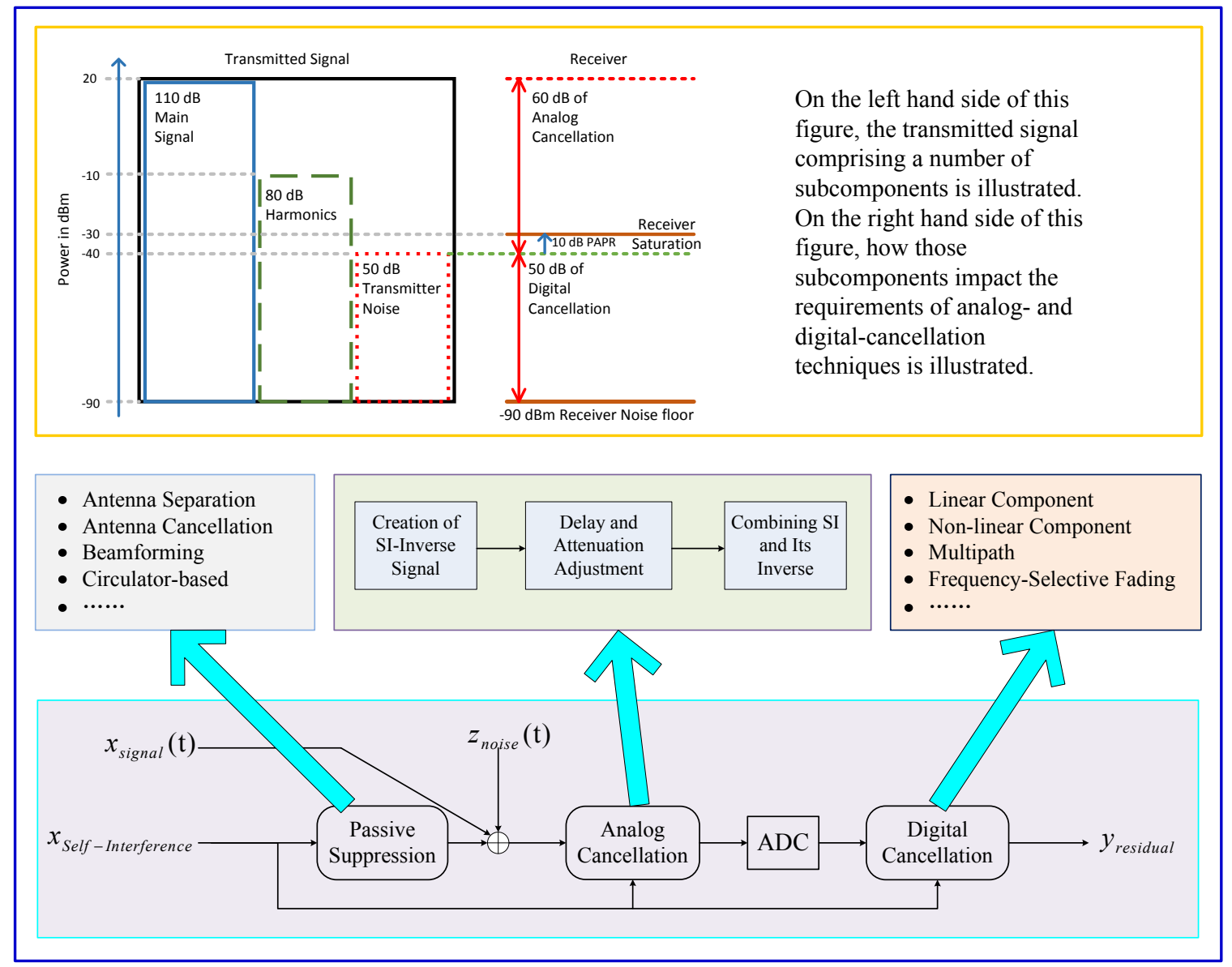

Fig. 1. To provide a sufficiently high SI cancellation capability, a FD radio must be capable of cancelling $110 \mathrm{~dB}$ of linear component, $80 \mathrm{~dB}$ of non-linear component as well as $60 \mathrm{~dB}$ of analog cancellation (Fig. 2 of [11]). Thus, the SI cancellation techniques can be classified into passive- and active-suppression, in which the latter technique can be further divided into analog and digital cancellations.

the ADC resolution. Explicitly, the FD devices must be capable of providing $60 \mathrm{~dB}$ of analog-domain cancellation plus $50 \mathrm{~dB}$ of digital-domain cancellation in order to reduce the SI to the receiver noise floor. However, if by any chance the analog- and/or digital-domain cancellations suffer from some performance degradations due to hardware imperfections and/or other impairments, their combined cancellation may not meet the decoding requirement. To mitigate the above-mentioned requirements as well as to mitigate the analog-/digital-domain requirements, a method referred to as passive suppression [7] can also be invoked for reducing the SI prior to reaching the RAs by exploiting the path-loss effect between the TAs and RAs of an FD node. 


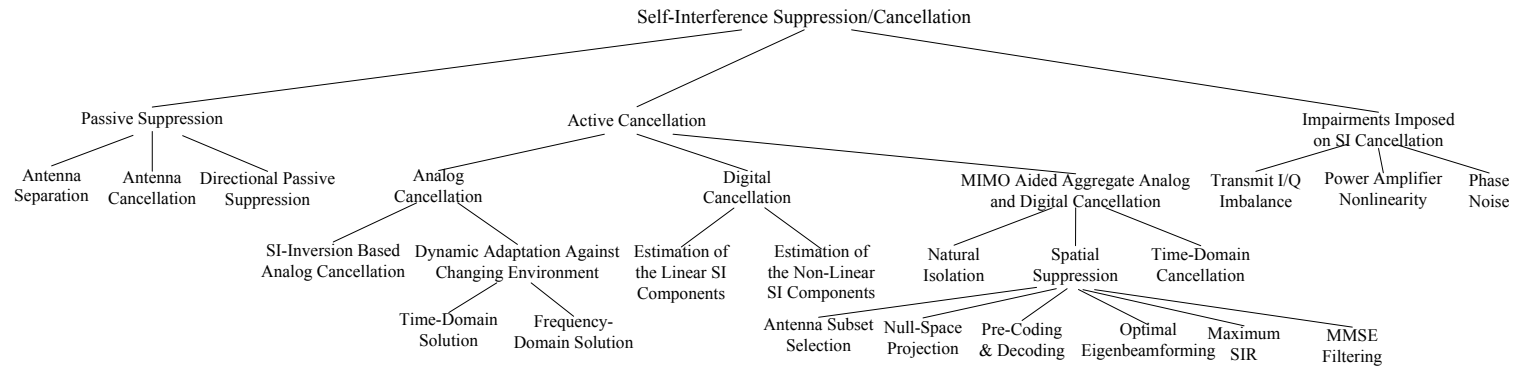

Fig. 2. Techniques related to SI measurement and suppression.

In this section, the SI cancellation techniques are classified into passive suppression, analog cancellation and digital cancellation, as described in Fig. 1. The family-tree of SI related techniques is seen in Fig. 2. According to the order of execution of different SI suppression/cancellation modules, we will first introduce the passive suppression techniques in the next subsection, followed by analog and digital cancellations.

\section{A. Passive Self-Interference Suppression}

Passive SI suppression is defined as the attenuation of the SI signal contributed by the path-loss effect due to the physical separation/isolation between the TAs and RAs of the same node [8]. By reducing the electromagnetic coupling between the TAs and RAs at the FD node, the power of SI can be reduced prior to its arrival at the RAs, as illustrated in Fig. 1. Numerous methods of passive SI suppression exist [12], [13], [59], [60]. For example, in a multi-antenna based system, the polarization decoupling technique enables the TAs and RAs to operate with the aid of orthogonal horizontal and vertical polarizations for the sake of reducing their coupling [60]. Furthermore, passive suppression may rely on beamforming-aided techniques for directing the lobes of TAs and RAs in different directions [13], hence resulting in improved physical separation between the TAs and RAs [12]. Additionally, by employing isolation components such as circulator-like devices, the transmit and receive paths of a single FD antenna can also be isolated, providing an equivalent SI-attenuation effect [59].

In the rest of this subsection, various passive suppression techniques will be surveyed and compared. The major contributions on passive suppression techniques are summarized in Table IV.

1) Antenna Separation Based Passive Suppression: The simplest method for achieving passive suppression might be resorted to the Antenna Separation (AS) technique, because in practice systems increasing the pathloss effect between the TAs and RAs constitutes an effective approach to attenuate the SI signal. Consider a system, in which each node is equipped with a TA and RA, a larger TA-RA separation implies having a higher SI suppression capability. In [63], Haneda et al., studied outdoor-to-indoor communication system operating at the center frequency of $2.6 \mathrm{GHz}$, where compact relay antenna was developed for serving as a signal repeater between the outdoor base stations (BSs) and indoor users. In this compact relay station the TAs and RAs are attached to the opposite sides of the physical construction for FD operation, whilst facilitating both the measurement and suppression of the SI. The results revealed that the isolation between the TAs and RAs measured in a multipath environment was $48 \mathrm{~dB}$ for the compact relay antenna used and this could be improved by further separating the TAs-RAs, while simultaneously optimizing the orientation of the antenna arrays. Furthermore, it was also shown that a $70 \mathrm{~dB}$ isolation can be achieved for a TAs-RAs separation of $5 \mathrm{~m}$, while ensuring the best possible antenna orientation. Although this isolation level may still be insufficient for practical FD operation, especially for compact relays, the employment of an interference canceller is capable of further increasing the amount of SI cancellation.

2) Antenna Cancellation Based Passive Suppression: The basic philosophy of antenna cancellation (AC) is to employ two TAs and a single RA, where the pair of TAs is placed at distances of $d$ and $(d+\lambda / 2)$ away from the RA, respectively, with $\lambda$ representing the wavelength [7]. The RA is positioned by satisfying that its distance from the TAs differs by an odd multiple of $\lambda / 2$, which results in the transmit signals being destructively superimposed for cancelling one another, as illustrated in Fig. 3. The destructive interference becomes most effective if the signal powers impinging at the RA from the pair of TAs are identical, thus (in theory) creating a null at the position of the RA. It has been shown in [7] that antennaaided cancellation techniques are capable of achieving an SI suppression of about $30 \mathrm{~dB}$, and in conjunction 
TABLE IV

Major Passive Suppression TeChNiques

\begin{tabular}{|c|c|c|c|c|}
\hline Year & Author(s) & Contribution & Complexity & Assumption \\
\hline 2004 & $\begin{array}{l}\text { Anderson } \\
\text { et al. [61] }\end{array}$ & $\begin{array}{l}\text { Presents antenna isolation approach for } \\
\text { FD MIMO aided relays. With separated } \\
\text { TA and RA arrays, natural isolation may } \\
\text { exploit surrounding buildings or add a } \\
\text { shielding plate. }\end{array}$ & High & $\begin{array}{l}\text { Both the strength and delay characteristics } \\
\text { of SI leakage-channel components are as- } \\
\text { sumed to be attainable. }\end{array}$ \\
\hline 2007 & Bliss et al. & $\begin{array}{l}\text { Performs antenna partitioning in the FD } \\
\text { MIMO aided relay to let some of the relay } \\
\text { antennas transmit while at the same time } \\
\text { let the other antennas receive. }\end{array}$ & Low & $\begin{array}{l}\text { Adaptive transmit and receive antenna ar- } \\
\text { ray approaches are assumed. }\end{array}$ \\
\hline 2009 & Ju et al. [9] & $\begin{array}{l}\text { Uses the same antenna array for both } \\
\text { reception and transmission to make all } \\
\text { natural isolation come solely from the } \\
\text { duplexer connecting the input and output } \\
\text { feeds to the same physical antenna ele- } \\
\text { ment. }\end{array}$ & Medium & $\begin{array}{l}\text { SI cancellation can be performed by using } \\
\text { precoding. }\end{array}$ \\
\hline \multirow[t]{3}{*}{2010} & $\begin{array}{l}\text { Duarte } \\
\text { al. }[12]\end{array}$ & $\begin{array}{l}\text { Increases the path-loss relying on antenna } \\
\text { separation. Algorithms such as AS-Only, } \\
\text { ASDC, ASAC and ASADC are proposed. }\end{array}$ & Medium & $\begin{array}{l}\text { Antenna separation can be up to } 20- \\
40 \mathrm{~cm} \text {. }\end{array}$ \\
\hline & Choi et al. & $\begin{array}{l}\text { Proposes a technique referred to as An- } \\
\text { tenna Separation to attenuate the received } \\
\text { SI that caused by two TAs separated by } \\
\text { an odd multiple of } \lambda / 2 \text {. }\end{array}$ & High & $\begin{array}{l}\text { Antenna cancellation considering ideal } \\
\text { antenna placement. }\end{array}$ \\
\hline & $\begin{array}{l}\text { Haneda } \\
\text { al. [63] }\end{array}$ & $\begin{array}{l}\text { Studies the Antenna Separation based pas- } \\
\text { sive suppression technique. }\end{array}$ & Medium & $\begin{array}{l}\text { The measurement of loop-back interfer- } \\
\text { ence channels can be performed at the } \\
\text { compact relay. }\end{array}$ \\
\hline 2011 & $\begin{array}{l}\text { Everett } \\
\text { al. [13] }\end{array}$ & $\begin{array}{l}\text { Exploits directional diversity technique, in } \\
\text { which the main lobs of TAs and RAs on } \\
\text { the FD node have minimal intersection. }\end{array}$ & Medium & $\begin{array}{l}\text { Directional antennas at } 10-15 \mathrm{~m} \text { distance } \\
\text { and with } 12 \mathrm{dBm} \text { transmit power. }\end{array}$ \\
\hline \multirow[t]{5}{*}{2012} & $\begin{array}{l}\text { Duarte et } \\
\text { al. [8] }\end{array}$ & $\begin{array}{l}\text { Defines the passive suppression as an at- } \\
\text { tenuation caused by path-loss made pos- } \\
\text { sibly by separation between the TAs and } \\
\text { RAs on the same node. }\end{array}$ & Medium & $\begin{array}{l}\text { A constant SIR at the receiver antenna is } \\
\text { assumed. }\end{array}$ \\
\hline & $\begin{array}{l}\text { Khandani } \\
\text { et al. [64] }\end{array}$ & $\begin{array}{l}\text { Uses decoupled antenna, in which dipole } \\
\text { antennas are placed in planes perpendic- } \\
\text { ular to one another, to minimize mutual } \\
\text { coupling. }\end{array}$ & Medium & $\begin{array}{l}\text { Mutual coupling can be minimized by } \\
\text { employing decoupled antennas. }\end{array}$ \\
\hline & $\begin{array}{l}\text { Everett } \\
\text { al. [60] }\end{array}$ & $\begin{array}{l}\text { Uses polarization decoupling, in which } \\
\text { the TAs and RAs operate on orthogonal } \\
\text { polarizations, to reduce the coupling and } \\
\text { improve the capability of passive suppres- } \\
\text { sion. }\end{array}$ & Medium & $\begin{array}{l}\text { Antenna architecture design by combining } \\
\text { directional isolation, absorptive shielding } \\
\text { and cross-polarization. }\end{array}$ \\
\hline & Knox et al. & $\begin{array}{l}\text { Uses circular-isolation, in which the trans- } \\
\text { mit and receive paths of a single FD } \\
\text { antenna is isolated via a circular/duplexer } \\
\text { to facilitate a high-performance passive } \\
\text { suppression. }\end{array}$ & Medium & $\begin{array}{l}\text { Achieves FD communications using a } \\
\text { common carrier operating with a single } \\
\text { antenna. }\end{array}$ \\
\hline & $\begin{array}{l}\text { Duarte et } \\
\text { al. [4] }\end{array}$ & $\begin{array}{l}\text { Demonstrates that the sum total of analog } \\
\text { and passive suppression does not increase } \\
\text { linearly with increase in passive suppres- } \\
\text { sion. }\end{array}$ & High & $\begin{array}{l}\text { The design is implemented at a } 20 \mathrm{MHz} \\
\text { MIMO OFDM system with a } 2.4 \mathrm{GHz} \\
\text { central frequency. }\end{array}$ \\
\hline
\end{tabular}

with existing RF interference cancellation [25] as well as digital baseband SI cancellation [17], [65], a cancellation capability as high as $60 \mathrm{~dB}$ can be achieved. Furthermore, as compared to traditional HD techniques, a median gain of $84 \%$ in aggregate throughput may be attained for a single-hop wireless channel by invoking AC mechanism [7].

3) Directional Passive Suppression: Directional SI suppression constitutes a technique, where the main radiation lobes of the TAs and RAs of an FD node have minimal intersection [13]. When performing passive suppression relying on the above-mentioned technique in a cellular system, the base station first invokes RF cancellation using the mechanism proposed in [15].
Hence the SI is partially suppressed prior to its arrival at the receiver's RF front-end. The performance of the directional SI suppression method was evaluated in [13] using the following experimental parameters:

- The method operates at a center frequency of $2.048 \mathrm{GHz}$;

- A wideband OFDM signal having a $20 \mathrm{MHz}$ bandwidth and 64 subcarriers is considered;

- A transmit power of $12 \mathrm{dBm}$ is assumed;

- The antennas have a $5 \mathrm{~dB}$ gain and $85^{\circ}$ half-power angular bandwidth.

The experimental results of [13] demonstrated that the FD mode significantly outperforms the HD mode, when 


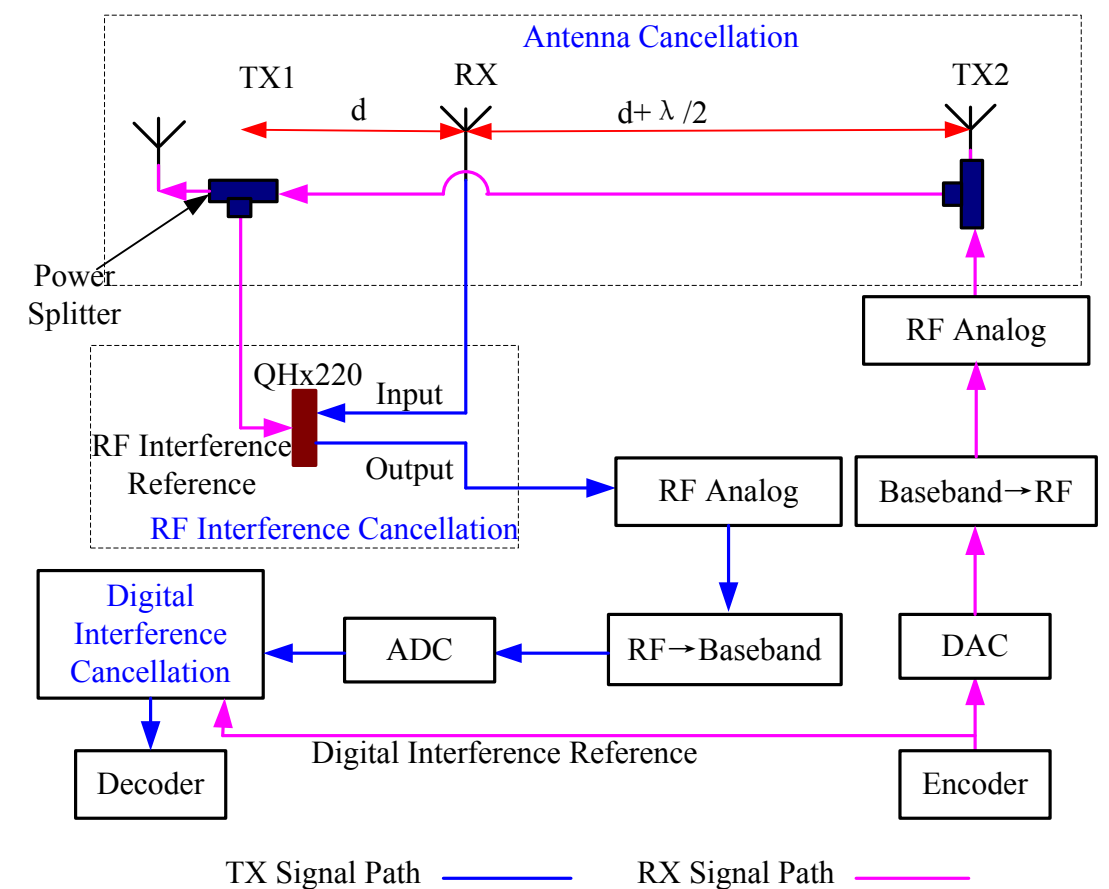

Fig. 3. Block diagram of antenna cancellation for a wireless FD SI cancellation. The power splitters introduce a $6 \mathrm{~dB}$ reduction in signal, thus power from TX1 is $6 \mathrm{~dB}$ lower compared to power from TX2, without the need for an additional attenuator to compensate for the amplitude mismatch (Fig. 2 of [7]).

relying on passive SI suppression combined with active SI cancellation. In a scenario, where the TA-RA distance is assumed to be $10 \mathrm{~m}$ and the antennas are separated by an angle of $45^{\circ}$ or more, the FD gain ${ }^{5}$ over the HD mode may range from $60 \%$ to $90 \%$. This gain becomes $50 \%$ or more for an antenna distance of $15 \mathrm{~m}$ and for an angle ranging from $90^{\circ}$ to $150^{\circ}$.

4) Open Research Issues in Passive Suppression: Although passive SI suppression techniques are capable of attenuating the SI signal in proportion to the pathloss, a larger TA-RA separation usually requires a higher device size, which may not be always feasible in practical systems. There are still multiple open challenges in implementing passive suppression, mainly reflected in the following aspects:

- The amount of SI reduction offered by the passive suppression technique itself is insufficient for flawless decoding. For instance, in the experiments performed in [12], antenna distances of $20 \mathrm{~cm}$ and $40 \mathrm{~cm}$ are considered. These separations are feasible for devices such as personal computers, but are actually insufficient for attenuating the interfering

${ }^{5}$ The FD gain as compared to the HD mode can be evaluated in terms of data rate, capacity, BER, and outage probability improvement, etc. power to a level below the power of the signal of interest;

- The SI-reduction capability of some passive suppression techniques (e.g. directional SI suppression and beamforming) may rely heavily on the multipleantenna configurations of the FD devices, preventing the size-limited receivers from sufficiently suppressing the SI power;

- Increasing the TA-RA separation may not always benefit the FD operation due to the following reasons:

- Increasing the TA-RA separation will degrade the estimate of the wireless channel between the TA and RA, consequently eroding both the SI channel's estimate and the resultant suppression;

- For some of the separation-sensitive techniques, a separation beyond the optimum distance (e.g. an odd multiple of $\lambda / 2$ specified in AC based suppression [7]) may even deteriorate the attainable cancellation;

- Furthermore, some passive suppression techniques may be band-limited, thus substantially eroding the cancellation performance of a wideband system. For 
example, it was found that the bandwidth of the transmitted signal imposes a fundamental constraint on the performance of AC based techniques [7]. Basically, the AC based technique only ensures at the central frequency that the signal is perfectly phase-inverted and cancelled. However, the perfect antenna positions derived for a specific frequency are no longer perfect for the other frequencies. Hence the $\mathrm{AC}$ based technique fails to provide perfect phase inversion at the RA position across the entire bandwidth. Specifically, in wideband OFDM systems, the SI cancellation may fail on a per subcarrier basis due to the channel's frequency selectivity.

In brief, the best antenna configuration in terms of the attainable SI suppression relies on installing the TAs and RAs at the opposite sides of the device in order to create the highest possible separation [15]. However, optimizing the antenna configuration of compact devices remains challenging. Hence, we have to resort to a combination of passive and active suppression/cancellation techniques for facilitating a better SI reduction in practically FD systems.

\section{B. Analog Self-Interference Cancellation}

Based on the above-mentioned discussion, we suppose that the amount of SI reduction relying on the pure passive suppression technique is insufficient for supporting high-integrity FD reception ${ }^{6}$. In order to reduce the SI below the noise level, we have to additionally invoke active cancellation techniques for further reducing the residual SI after passive suppression. Hence, the objective of the additional SI cancellation modules is to minimize the SI either within the RF [7] or in the analog/digital baseband stage.

In theory, the employment of the RF/analog cancellation module is not mandatory, if the FD radio is capable of performing a perfect SI leakage-path estimation at the RAs, the reconstructed digital samples of the SI signal may be readily subtracted from the low-power received samples, for example by using techniques such as ZigZag decoding [17]. Unfortunately, a strong SI signal would saturate the AGC, which is hence desensitized for the reception of a weak desired signal compressed to a range much smaller than $[-1,1]$. In this case, the ADC that becomes impact of the extremely strong

\footnotetext{
${ }^{6}$ The signal received at the RAs will be first amplified by an AGC and then down-converted to the baseband/intermidiate frequency, followed by filtering and sampling before the ADC to create the digital samples.
}

SI power. More explicitly, the quantization noise contaminating the desired signal might become excessive, hence resulting in a negative effective SINR that would become inadequate for recovering the desired signal in the digital baseband [10]. As indicated by [66], the limitations of the ADC, such as its estimated dynamic range and quantization resolution constitute the main obstacle in improving the achievable SI-isolation levels by employing digital cancellation.

Therefore, it is critical to further reduce the power of the SI signal prior to the digitization ${ }^{7}$ of the desired received signal. Specifically, a mechanism referred to as analog cancellation has to be invoked for mitigating the SI contaminating the analog signal before it is digitized. After performing analog cancellation, the decontaminated received digital samples will exhibit a sufficiently high resolution of the desired received signal, thus facilitating efficient digital SI cancellation [7], as depicted in Fig. 1. In this subsection, a range of beneficial analog cancellation techniques will be surveyed and compared, followed by the family of digital cancellation techniques. For the reader's convenience, we have summarized the seminal contributions on the subject of analog/digital cancellation techniques in Table V.

1) Analog Cancellation for Reducing the Linear SI Component: In this section, we focus our attention on the fundamentals of analog cancellation by elaborating on the reduction of the linear SI component, which constitutes the majority of the SI power, leaving the dynamic adaptation based solutions guarding against the non-linear components encountered in time-variant environments for further study in the next part.

The principle of analog cancellation can be simply summarized as follows: In order to sufficiently reduce the SI power, an FD radio is required for creating a reference signal corresponding to a perfect replica of the SI signal at all instances. Combining this replica and the SI signals is in theory capable of facilitating perfect SI cancellation [10]. Basically, the analog cancellation can be performed either at the RF or at the analog baseband stage [29]. However, most of the existing analog cancellation (e.g. [7], [10], [12]) techniques operate at the RF. Furthermore, by identifying whether the perfect replicabased SI cancelling signal is generated by processing the SI prior to or after up-conversion, the RF-based analog cancellation arrangements may be further classified as pre-mixer (e.g. [12]) or post-mixer schemes (e.g. [10]). The baseband analog canceller, on the other hand, is defined as the canceller, in which the perfect replica-

\footnotetext{
${ }^{7}$ Before performing digitization, the AGC scales the input to the normalized range of $[-1,1]$.
} 
TABLE V

Major AnAlog And Digital CANCELlation TECHNiQUES

\begin{tabular}{|c|c|c|c|c|}
\hline Year & Author(s) & Contribution & Complexity & Assumption \\
\hline 2008 & $\mathrm{Ju}_{\text {[6] }}$ et al. & $\begin{array}{l}\text { Proposes antenna-sharing-based method } \\
\text { for FD MIMO aided relays. }\end{array}$ & Medium & $(2 \times 2 \mathrm{MIMO}$ is considered.) \\
\hline \multirow[t]{4}{*}{2009} & $\begin{array}{l}\text { Riihonen et } \\
\text { al. [68] }\end{array}$ & $\begin{array}{l}\text { Proposes a null space projection method } \\
\text { using ZF filters to make the MIMO re- } \\
\text { lay receive and transmit in different sub- } \\
\text { spaces. }\end{array}$ & Medium & $\begin{array}{l}\text { The loop-interference channel is esti- } \\
\text { mated at the FD MIMO relay. }\end{array}$ \\
\hline & $\mathrm{J}_{[69]}$ et $a l$. & $\begin{array}{l}\text { Eliminates SI at MIMO relays by exploit- } \\
\text { ing antenna selection diversity. }\end{array}$ & Low & $\begin{array}{l}\text { Two-way and FD relaying systems with } \\
\text { ideal SI cancellation. }\end{array}$ \\
\hline & $\begin{array}{l}\text { Radunovic } \\
\text { et al. [14] }\end{array}$ & $\begin{array}{l}\text { Proposes RF interference cancellation for } \\
\text { indoor FD wireless systems. }\end{array}$ & Low & $\begin{array}{l}\text { Advocate FD networking in a single band } \\
\text { with low power. }\end{array}$ \\
\hline & Chyun et al. & $\begin{array}{l}\text { Proposes a pre-nulling method using the } \\
\text { estimate of the interference channel to } \\
\text { facilitate pre-processing for the SI reduc- } \\
\text { tion. }\end{array}$ & Medium & Flat-fading channel is assumed. \\
\hline \multirow[t]{4}{*}{2010} & $\begin{array}{l}\text { Duarte } \\
\text { al. }[12]\end{array}$ & $\begin{array}{l}\text { Proposes a parallel radio cancellation } \\
\text { through cancelling signal, which is the } \\
\text { negative of the SI signal. }\end{array}$ & Medium & Operate at $2.4 \mathrm{GHz}$. \\
\hline & Choi et al. & $\begin{array}{l}\text { Proposes antenna cancellation method to } \\
\text { use two TAs and one RA, with the two } \\
\text { TAs placed at distances } d \text { and } d+\lambda / 2 \\
\text { away from the RA. }\end{array}$ & High & $\begin{array}{l}\text { Two transmit antennas are separated by an } \\
\text { odd multiple of } \lambda / 2 \text {. }\end{array}$ \\
\hline & Chun et al. & $\begin{array}{l}\text { Proposes a spatial nullification method } \\
\text { based on an one-step operation for FD AF } \\
\text { MIMO relays. }\end{array}$ & High & Zero delay at the FD AF relay is assumed. \\
\hline & $\begin{array}{lll}\text { Lee } \\
{[70]}\end{array}$ et al. & $\begin{array}{l}\text { Proposes } \mathrm{ZF} \text { beamforming for FD MIMO } \\
\text { aided relays based on block diagonalisa- } \\
\text { tion approach. }\end{array}$ & High & $\begin{array}{l}\text { Channel coefficients follow i.i.d. complex } \\
\text { Gaussian distribution with zero mean and } \\
\text { unit variance. }\end{array}$ \\
\hline \multirow[t]{6}{*}{2011} & Jain et al. & $\begin{array}{l}\text { Proposes the post-miser cancellers, which } \\
\text { generate cancelling signal by processing } \\
\text { after SI is up-converted. }\end{array}$ & High & $\begin{array}{l}\text { Two-antenna FD device operates at } \\
10 \mathrm{MHz} \text { WiFi signal. }\end{array}$ \\
\hline & $\begin{array}{l}\text { Everett et } \\
\text { al. [71] }\end{array}$ & $\begin{array}{l}\text { Uses time-orthogonal training to facilitate } \\
\text { a structured SI cancellation in the time } \\
\text { domain. }\end{array}$ & Low & $\begin{array}{l}\text { The relay decoder has a priori knowledge } \\
\text { of the interference sequence. }\end{array}$ \\
\hline & $\begin{array}{l}\text { Sahai et al. } \\
\text { [15] }\end{array}$ & $\begin{array}{l}\text { Performs an active analog SI cancellation } \\
\text { by injecting an appropriate scaled can- } \\
\text { celling signal before the received signal } \\
\text { reaches the ADC. }\end{array}$ & Medium & $\begin{array}{l}\text { The FD system operates at a real-time 64- } \\
\text { subcarrier OFDM signal with bandwidth } \\
\text { of } 10 \mathrm{MHz} \text {. }\end{array}$ \\
\hline & $\begin{array}{l}\text { Sung et al. } \\
{[21]}\end{array}$ & $\begin{array}{l}\text { Proposes Transmit Antenna Selection } \\
\text { (TAS) to obtain a high diversity gain with } \\
\text { a low complexity. }\end{array}$ & High & $\begin{array}{l}\text { The number of antennas at source is no } \\
\text { more than that of the transmit/receive an- } \\
\text { tennas at the relay. }\end{array}$ \\
\hline & $\begin{array}{l}\text { Riihonen et } \\
\text { al. [19] }\end{array}$ & $\begin{array}{l}\text { Uses methods of null space projection and } \\
\text { MMSE filters to perform SD suppression. }\end{array}$ & High & $\begin{array}{l}\text { The FD relay can eliminate the } S I \text { in the } \\
\text { ideal case with perfect side information. }\end{array}$ \\
\hline & $\begin{array}{l}\text { Riihonen et } \\
\text { al. [72] }\end{array}$ & $\begin{array}{l}\text { Proposes optimal eigenbeamforming } \\
\text { method to minimize the SI power. }\end{array}$ & High & $\begin{array}{l}\text { The SI leakage-channels are assumed to } \\
\text { remain time-invariant during each symbol } \\
\text { period. }\end{array}$ \\
\hline \multirow[t]{2}{*}{2012} & $\begin{array}{l}\text { Lopez et al. } \\
{[73]}\end{array}$ & $\begin{array}{l}\text { Implements the active feedback cancella- } \\
\text { tion for an FD relay with multiple receive } \\
\text { and one TA. }\end{array}$ & High & $\begin{array}{l}\text { Operate at } 8 \mathrm{MHz} \text { OFDM signal with a } \\
\text { carrier frequency of } 842 \mathrm{MHz} \text {. }\end{array}$ \\
\hline & Chun et al. & $\begin{array}{l}\text { Performs enhanced joint-nulling scheme } \\
\text { to simultaneously mitigate the SI and } \\
\text { maximize the ergodic rate. }\end{array}$ & High & $\begin{array}{l}\text { The SI power is at a level from } 10 \text { to } \\
50 \mathrm{dBm} \text {. }\end{array}$ \\
\hline \multirow[t]{2}{*}{2013} & $\begin{array}{l}\text { Stankovic } \\
\text { et al. }[75]\end{array}$ & $\begin{array}{l}\text { Transforms the FD MIMO aided relaying } \\
\text { channel into a block diagonal matrix by } \\
\text { using the low-complexity block DFT. }\end{array}$ & Medium & $\begin{array}{l}\text { The block circulant MIMO channel can be } \\
\text { transformed into a block diagonal matrix } \\
\text { using a block DFT. }\end{array}$ \\
\hline & $\begin{array}{l}\text { Bharadia et } \\
\text { al. [11] }\end{array}$ & $\begin{array}{l}\text { Proposes a circulator based single-antenna } \\
\text { FD platform, and eliminates linear/non- } \\
\text { linear SI components using digital cancel- } \\
\text { lation. }\end{array}$ & High & $\begin{array}{l}\text { A single-antenna FD device can be imple- } \\
\text { mented relying on a circulator. }\end{array}$ \\
\hline 2014 & $\mathrm{Li}_{[76]}$ et al. & $\begin{array}{l}\text { Proposes two digital cancellation tech- } \\
\text { niques, i.e. cancellation based on the out- } \\
\text { put signal of power amplifier and two- } \\
\text { stage iterative cancellation. }\end{array}$ & Medium & $\begin{array}{l}\text { The effects of ADC, phase noise and sam- } \\
\text { pling jitter are no longer bottleneck after } \\
\text { performing RF/analog SI cancellation. }\end{array}$ \\
\hline
\end{tabular}


based cancelling signal is generated in the baseband and the cancellation occurs in the analog baseband [29].

Based on the above-mentioned principle, the operation of analog cancellation can be realized by executing the following three steps, including:

- Creation of SI-Inverse Signal: Basically, SI inversion can be implemented by an FD radio upon simply inverting a signal by inverting its phase. However, this phase adjustment may only be feasible across a limited bandwidth, which hence limits its maximum cancellation capability. In other words, a perfect signal inversion can be attained at the central frequency, but the inverted signals will deviate at both sides of the central frequency from $180^{\circ}$, hence suffering from a significant phase-distortion. To address the above-mentioned problem, we have to resort to sophisticated hardware/circuit design relying on

- A balanced/unbalanced (balun) transformer, which is a common component in RF, audio and video circuits, can be utilized for perfectly (in theory) converting back and forth between an input signal and its inverse at all instances [10]. As illustrated in Fig. 4, the TA is assumed to transmit the positive signal. The balun output of RF reference, which is subject to an adjustment on the delay and attenuation of the reference signal, highly matches the SI signal at the RA, thus offering a reliable SI nulling by combining the received SI signal with its negative version.

- Apart from that, another method of generating the RF reference signal is to view the SI cancellation as a sampling and interpolation problem, which can be resolved relying on the delay-line based analog circuit [11]. By picking up the phase and amplitude of the SI signal (e.g. relying on Nyquist sampling theorem [77]), we can always reconstruct the SI signal at any instant as a weighted linear combination of samples taken before and after the recreation instant, with the weights of the linear combination determined by using the so called since interpolation algorithm. Of course, a fundamental tradeoff between the hardware complexity (i.e. in terms of the number of delay lines) and the cancellation capability must be treated.

- Delay and Attenuation Adjustment: Since the signal transmitted over the ether experiences both attenuation and delay in all practical scenarios, an identical attenuation and delay has to be applied to the inverted SI. The QHx220 noise cancellation chip [78], separates the SI-inversion-based RF reference signal into its in-phase and quadrature components (i.e. $g_{i}$ and $g_{q}$ ), can be invoked for imposing an adaptively controllable delay on the aggregated output signal by carefully controlling the attenuation of those components. It is shown in [10] that the balun-aided cancellation is capable of achieving an impressive SI reduction across a wide bandwidth, provided that both the phase and the amplitude of the inverted SI signal are set appropriately.

- Creating a SI-Null by Combining the SI and Its Inverse: The SI-inverse signal will then be combined with the SI signal at the RA. Without loss of generality, the Received Signal Strength Indicator (RSSI) values can be employed for representing the residual SI energy remaining after combining, as illustrated in Fig. 4. In theory, a perfect SI-inverse signal will result in zero SI value at the output of the RSSI. However, the realistic practical engineering imperfections of the hardware components, such as power leakage or a non-flat ${ }^{8}$ frequency response at balun, will always result in a residual SI power ${ }^{9}$ after signal combining, which can be minimized by carefully adapting the attenuations $\left(g_{i}\right.$ and $\left.g_{q}\right)$ using self-tuning algorithms [10].

The existing studies have demonstrated that analog cancellation techniques are capable of reducing the SI strength by dozens of dBs [7], [10], [11]. For example, in order to facilitate FD communication at the transmit powers typical of WiFi devices [13], it was shown experimentally [61] that the SI-induced contamination imposed on a modulated constellation point can be significantly reduced by using an interference canceller accomodated within the RF stage. Furthermore, as indicated in [10], the SI-inverse technique alone is capable of reducing the SI by no less than $45 \mathrm{~dB}$ across a $40 \mathrm{MHz}$ bandwidth.

Nonetheless, subtracting the SI from the received signal by simply relying on the above-mentioned SIinversion technique remains a challenge in practical systems, because the FD radio only knows the "clean" digital representation of the baseband signal, rather than

\footnotetext{
${ }^{8}$ For example, the balun circuit is not frequency flat and inverts different parts of the bands with different amplitudes, thus applying a single attenuation and delay factor to invert the SI signal will never achieve a perfect cancellation [10]. Furthermore, the QHx220 module may also suffer from a non-linear distortion, resulting in imperfect SI cancellation for typical wireless input powers $(0-30 \mathrm{dBm})[10]$.

${ }^{9}$ In practical designs, the combining-output energy can be further reduced in the digital-domain relying on digital cancellation techniques.
} 


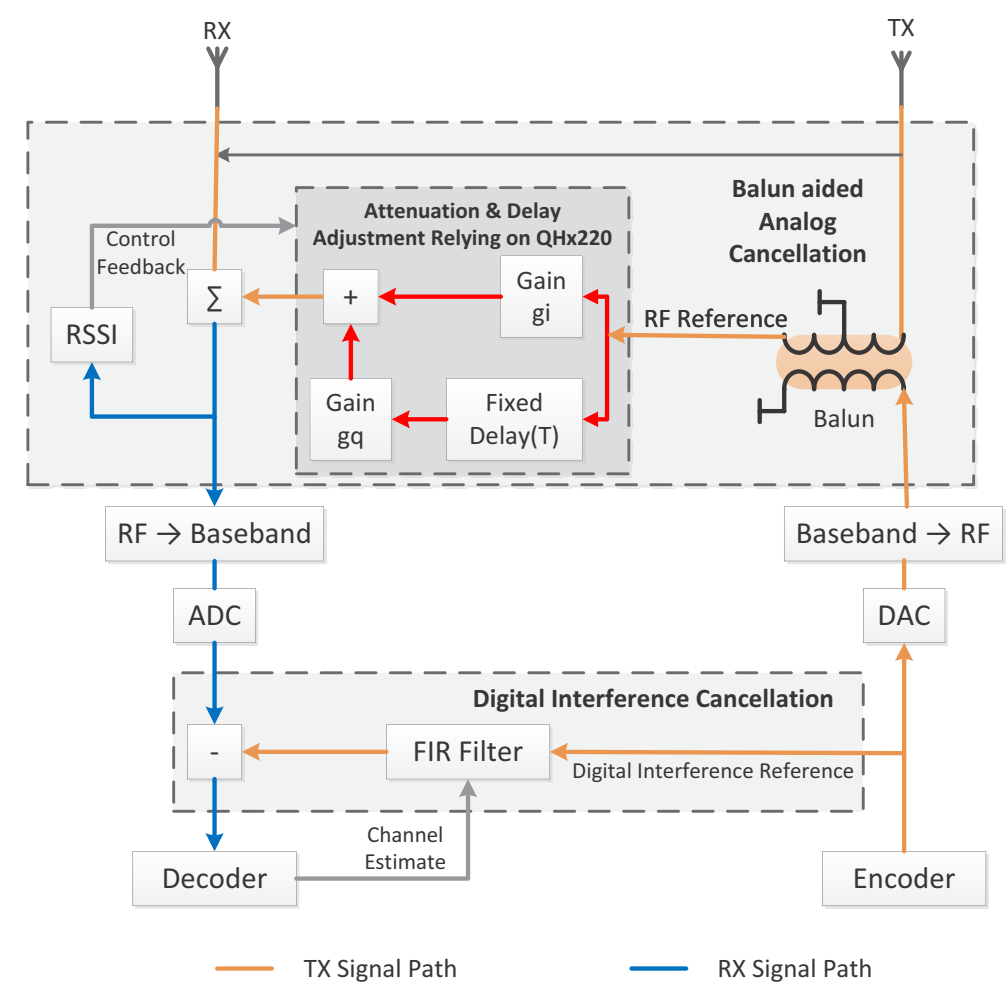

Fig. 4. Block diagram representation of analog and digital SI cancellations, in which the SI invert is executed by employing balun circuit, followed by QHx220 based delay \& attenuation adjustment. (Figs. 3 \& 7 in [10]).

its processed counterpart transmitted over the air. Once the signal is converted to the analog domain and upconverted to the carrier frequency for transmission, the transmitted signal becomes an unknown non-linear function of the ideal source signal contaminated by unknown distortions induced either by the imperfections of the analog components in the radio transmit chains (e.g. the third- and higher-order signal components created by the analog circuits, the transmitter noise due to the non-linearity of the power amplifiers, and the inaccuracy of the oscillators, etc) or by their non-flat frequency response [11]. In other words, the SI cancellation circuits that simply subtract the estimate of the transmit signal without taking into account all the non-linear distortions fail to perfectly cancel the SI by reducing it below the noise level. As indicated by [11], no more than $85 \mathrm{~dB}$ of SI power reduction can be achieved by FD designs that fail to account for the non-linear distortions. To make up for the deficiencies of the above-mentioned techniques, the non-linear SI components induced either by hardware imperfections or by the time-variant environment has to be carefully considered in designing the analog cancellation circuits.
2) Dynamic Adaptation of Analog Cancellation to Remove Non-Linear SI Components in Time-Varying Environments: While the above-mentioned analog cancellation schemes are capable of effectively dealing with the linear SI components, a time-variant environment encountered in the presence of channel fading, transmit power and other parameter fluctuations may impose a significant non-linear distortion based contamination on the cancellation [11]. More importantly, as the environment changes, the cancellation capability may drop to an inadequate level, because the already-optimized SI cancellation parameters based on the past environmental conditions may no longer correctly model the current SI.

To avoid the above-mentioned imperfections and provide a satisfactory cancellation performance, the FD radio must be capable of promptly tuning the analog circuit in order to adaptively respond to time-variant environments. Specifically, an adaptive scheme acting in response to the channel fluctuations must be conceived to equip the cancellation circuits with the capability of frequently and promptly refreshing its parameters (e.g. phase and amplitude of the SI-inverse-based RF reference signal) [10], [11]. In practical systems, we may 
invoke both the time- and frequency-domain solutions in order to combat the non-linear SI components in timevarying environment.

- Time-Domain Solutions: In [10], [11], a method for quickly tuning the analog circuit is proposed. For a given time-domain reference signal $c(t)$, the corresponding received SI signal $y(t)$ can be modelled as a summation of weighted reference samples at different delays, i.e. $\tilde{y}(t)=\sum_{i=1}^{N} \alpha_{i} c(t-$ $d_{i}$ ), where $N$ denotes the maximum number of taps, $\alpha_{1}, \cdots, \alpha_{N}$ each represents the attenuation corresponding to one delayed component, and $d_{1}, \cdots, d_{N}$ stand for delays associated with the taps, as shown in Fig. 5. The goal of the proposed tuning is to adaptively changing $\alpha_{1}, \cdots, \alpha_{N}$ such that the remaining SI power is minimized, i.e.

$$
\min _{\alpha_{1}, \cdots, \alpha_{N}}(y(t)-\tilde{y}(t))^{2} .
$$

The above-mentioned equation can be solved by using the so called Iterative Gradient Descent Algorithm [10]. Although this algorithm is simple, the extremely low convergence speed substantially constrains its practical application. It was shown in [11] that the algorithm requires nearly $40 \mathrm{~ms}$ to converge, which cost is pretty high for practical systems (i.e. corresponding to a $40 \%$ overhead in practical scenarios ${ }^{10}$ that require the analog cancellation to re-tune once every $100 \mathrm{~ms}$ ). Fortunately, this high tuning cost can be substantially reduced (i.e. to about $920 \mu \mathrm{s}$, as experimentally shown in [11]) by executing the initial settings of the attenuators relying on some known sequences such as the WiFi preamble, followed by finding the optimal convergence point after running a few gradientdescent iterations.

- Frequency-Domain Solutions: Unlike in (1), the frequency-domain SI signal can be modelled as a function of the tapped signal $c(t)$ as [11]:

$$
\mathbf{Y}(f)=\mathbf{H}(f) \mathbf{C}(f),
$$

where $\mathbf{H}(f)$ denotes the frequency-domain SI distortion induced by various factors such as the antenna, circulator and reflections, and $\mathbf{C}(f)$ represents the frequency-domain representation of the tapped signal (i.e. the discrete Fourier transform

\footnotetext{
${ }^{10}$ Note that the re-tune period is environment dependent. In [11], the "near field coherence time" of analog cancellation is defined to specify the time up to which the receiver remains unsaturated from the last time tune. This time duration can be used to trigger the return of the tuning algorithm.
}

(DFT) of $c(t)$ ). Similar to the time-domain solution, the frequency-domain SI-cancellation problem can be readily formulated as

$$
\min _{\alpha_{1}, \cdots, \alpha_{N}}\left(\mathbf{H}(f)-\sum_{i=1}^{N} \mathbf{H}_{\mathbf{i}}^{\boldsymbol{\alpha}_{i}}(f)\right)^{2},
$$

where $\mathbf{H}_{\mathbf{i}}^{\boldsymbol{\alpha}_{i}}(f)$ denotes the frequency response for attenuations $\alpha_{i}$. In theory, an exhaustive-search scheme can be implemented to make (3) converge to its optimal point. However, since achieving an optimal point is an NP hard problem, a substituted solution can be proposed by looking for a suboptimal point that enables the circuits to provide the required cancellation performance. It was shown experimentally in [11] that the convergence duration of the frequency-domain quick-tuning algorithm is no longer than $900-1000 \mu \mathrm{s}$, corresponding to less than $1 \%$ overhead for analog cancellation that performs re-tuning once every $100 \mathrm{~ms}$.

3) Open Research Issues in Analog Suppression: Based on the above-mentioned discussions, SI cancellation levels up to dozens of $\mathrm{dBs}$ may be achieved in broadband wireless channels by invoking analog cancellation techniques after performing passive suppression. However, there are still numerous further challenges to address in the context of analog cancellation techniques. For example, the costly hardware required for generating an accurate SI-inverse-based reference signal, the non-flat frequency response of the hardware and the dispersive and non-linear nature of the SI channel will all impose a performance limit on the analog cancellation capability. In the following, a number of possible solutions to the above-mentioned challenges will be introduced, followed by a range of potential research directions related to the field of analog cancellation.

- The fundamental tradeoff between the hardware costs and SI cancellation capability still constitutes a practical challenge: Requiring a higher cancellation capability implies stringent requirements on the precision of the hardware. For example, to achieve a $50 \mathrm{~dB}$ reduction of the SI requires the inverted SI signal to be within a normalized accuracy of $10^{-5}$ of the true SI signal, corresponding to a 99.999\% accuracy [10]. Furthermore, in the context of analog cancellation designs, such as the delayline based technique of [11], adopting more delayline stages implies offering a higher delay resolution accuracy, which is, however, attained at the cost of a more complex and large-size hardware circuits. How to effectively address the above-mentioned challenge and to develop a cost-efficient analog 


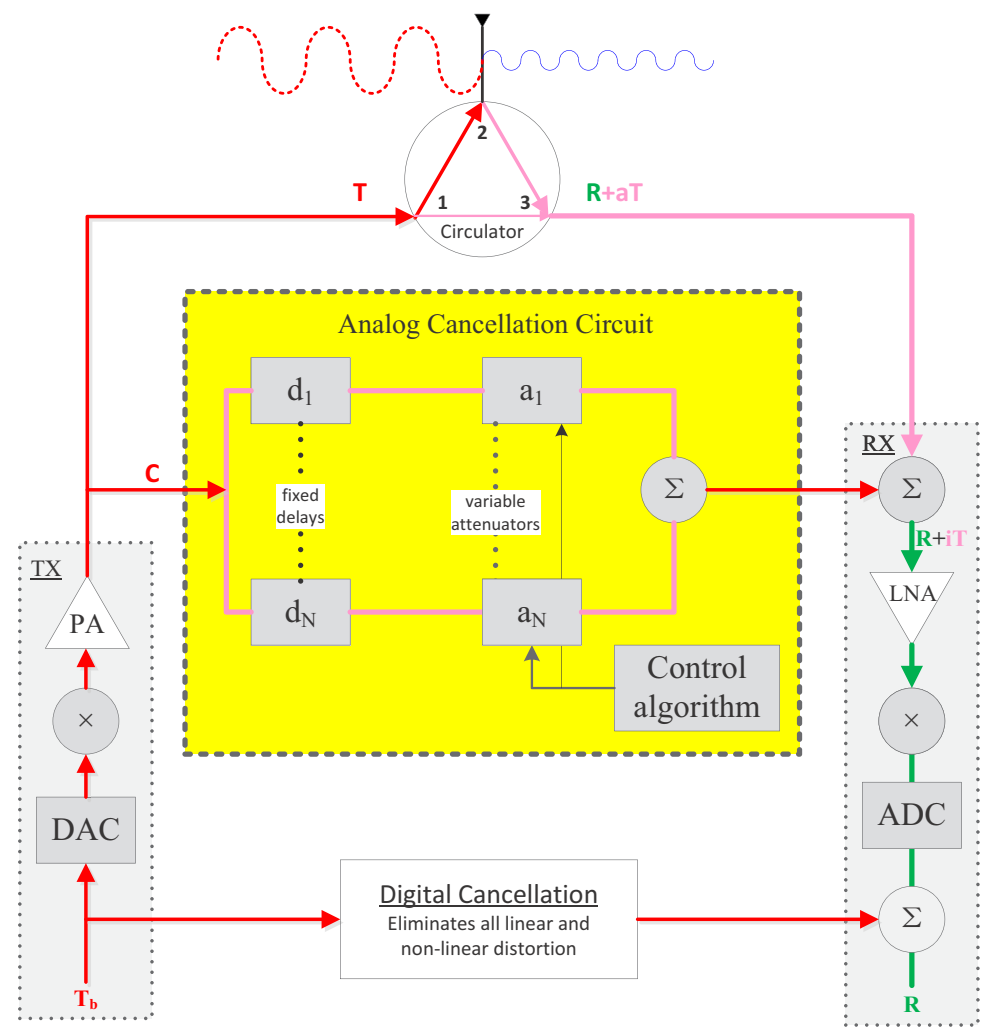

Fig. 5. Circulator aided FD radio block diagram, in which $T_{b}$ denotes the intended baseband signal for transmission, while $T$ is the practically transmitted RF signal. For an intended receive signal $R$, it will be contaminated by the strong components partially due to the undesirable leakage of the circulator. Both the static SI and the time-variant distortions will be combated by the analog cancellation circuit. Furthermore, in the digital domain, both the linear and non-linear components of the residual SI will be estimated and subtracted in the Digital Cancellation functional block (Fig. 3 in [11]).

cancellation circuit exhibiting a sufficiently high cancellation capability remains challenging at the time of writing.

- Hardware Imperfections Limit the SI Cancellation: In practical designs, the limited sensitivity and precision of the hardware components substantially constrain the attainable SI cancellation capability. For example, as emphasized in [10], the QHx220-aided cancellation will remain imperfect for typical input powers $(0-30 \mathrm{dBm})$, and the balun circuit exhibiting a non-flat frequency response ${ }^{11}$ will limit the maximum attainable cancellation. What deserves in-depth analysis is offering a sufficiently high cancellation capability of mitigating the linear/non-linear SI components relying on the $\mathrm{RF} /$ analog cancellation circuits, while additionally carefully considering the constraints imposed by the

\footnotetext{
${ }^{11}$ As mentioned in [10], this uneven frequency response may partially come from the RF echoes in the balun board.
}

hardware imperfections.

- Transmit Power Control for Improving the SI Suppression [7]: Although increasing the transmit power is beneficial in terms of improving the SI channel estimation, whilst reducing the ratio between the residual SI power and the overall SI strength, a higher transmit power inevitably increases the absolute level of the residual SI power. High transmit powers (e.g. beyond $20 \mathrm{dBm}$ ) would be very hard to cancel [7]. Furthermore, in order to facilitate a higher analog cancellation relying on techniques, such as in-line high-precision attenuation and delay circuits [11], the non-linear distortion inflicted by the cancellation circuits at high input powers must be addressed [10]. Further studies are thus needed for conceiving optimal SI cancellation in the context of a combined transmit power control framework, while relying on reduceddistortion hardware design. 
In summary, the achievable SI cancellation capability may remain limited and in fact insufficient for highintegrity detection, when relying on stand-alone analog cancellation. To offer a sufficiently high cancellation capability (i.e. to make the resultant SINR high enough for high-integrity detection), digital-domain cancellation combined with analog cancellation must be employed for further mitigating the residual SI in the digital baseband.

\section{Digital Self-Interference Cancellation}

As indicated by [10], although an industry-grade balun circuit is capable of reducing the SI by as much as $45 \mathrm{~dB}$ for a $40 \mathrm{MHz}$ wide SI signal, the remaining SI power may remain by up to $45 \mathrm{~dB}$ higher than the noise floor (in the absence of employing passive suppression). This still excessively interferes with the desired signal, either because of the residual multipath SI echoes contaminating the desired signal or because of the SI leakage imposed by the imperfections of the hardware circuits. Evidently, the residual SI after analog cancellation must be further reduced in the digital domain.

Digital cancellation constitutes an active SI-mitigation mechanism that by definition operates in the digital domain and exploits the knowledge of the interfering signal in order to cancel it after the received signal has been quantized by the ADC [29], [79]. To achieve this, the receiver first extracts the SI and then remodulates it and subtracts it from the received SI contaminated signal. Coherent SI-detection can also be employed for recovering the SI by correlating the received signal with the clean hypothesized regenerated SI-inversion based reference signal, which is available at the output of the co-located FD transmitter [7]. This technique then requires the receiver to estimate both the delay and phase shift between the transmitted and the received signals relying on techniques, such as the correlation peak based algorithm for subtracting the SI signal.

1) Fundamentals of Digital Cancellation: Digital cancellation can be regarded as an excellent, safety-net solution for diverse scenarios, when analog cancellation achieves a poor suppression [7], [12]. However, since the transmitted packet are different from the generated reference signal due to a number of factors such as the hardware limitations and the multipath fading, subtracting the estimated signal rather than the clean signal would be capable of substantially improving the capability of digital cancellation. In practice, digital cancellation fundamentally comprises two main components, i.e. estimating the SI channel, and using the channel estimation on the known transmit signal to generate digital samples for subtracting the SI from the received signal [10].
In order to implement the digital cancellation to eliminate the residual SI power after analog cancellation, the SI channel components comprising both the leakage over through the analog cancellation circuit and the delayed reflections of the SI signal from the environment must be estimated [11]. Basically, the residual SI can be sub-divided into linear and non-linear components. The former constitutes the majority of the SI power and can be estimated by existing algorithms, such as the family of least-square and MMSE [80] based techniques, while the latter is induced by the non-linear distortions of the imperfect analog cancellation circuits. For example, the QHx220 hardware [10] employed in the balunbased analog cancellation scheme may cause non-linear distortions, particularly for high input powers beyond say $-40 \mathrm{dBm}$ [10]. Consequently, the non-linearity of the SI leakage channel must be accurately characterized for the sake of high-rejection SI cancellation in the digital domain. In practical designs, we may rely on the following techniques for estimating the linear and nonlinear components [11]:

2) Estimation of the Linear Component of the SI Leakage-Channel: By modelling the linear components of the SI as a non-causal linear function of the transmitted digital signal $x[n]$, which is known in advance, the received sample $y[n]$ at any instant can be modelled as a linear combination of up to $k$ samples of $x[n]$ before and after the instant $n$, where $k>0$ is a function of the SI leakage channel memory:

$$
y[n]=\sum_{z=1-k}^{k} x[n-z] h[z]+w[n],
$$

where $h[n]$ and $w[n]$ represent the SI channel attenuation and the additive noise component at instant $n$, respectively. By defining $\boldsymbol{y}=[y[0], \cdots, y[n]]^{T}$, $\boldsymbol{h}=[h[-k], \cdots, h[0], \cdots, h[k-1]]^{T}$, and $\boldsymbol{w}=$ $[w[0], \cdots, w[n]]^{T}$, where $\boldsymbol{x}^{T}$ denotes the transpose of vector $\boldsymbol{x}$, the SI channel vector $\boldsymbol{h}$ can be estimated as follows [11]:

$$
\hat{\boldsymbol{h}}=\left(\boldsymbol{A}^{H} \boldsymbol{A}\right)^{-1} \boldsymbol{A}^{H} \boldsymbol{y}
$$

$\left.\begin{array}{ccccc}\text { where } & \text { we } & & \text { have } & \boldsymbol{A} \\ x[-k] & \ldots & x[0] & \ldots & x[k-1] \\ \vdots & \ddots & \vdots & \ddots & \vdots \\ x[n-k] & \ldots & x[n] & \ldots & x[n+k-1]\end{array}\right], \quad$ and $\boldsymbol{A}$. Since the training matrix $\boldsymbol{A}$ can be pre-computed, the computational complexity of the above-mentioned algorithm can be substantially reduced. 
3) Estimation of the Non-Linear Components of the SI Leakage-Channel: After estimating and eliminating the linear components of the SI signal, the residual nonlinear components can be further reduced. As indicated by [11], the power of the residual non-linear components is about $20 \mathrm{~dB}$ higher than the noise level. Since the exact non-linear function that an FD radio applies to the baseband transmitted signal is hard to estimate, a general model relying on Taylor series expansion can be employed for approximating the non-linear function in the digital baseband domain [11]:

$$
y[n]=\sum_{m \in \text { odd terms }, n=-k, \cdots, k} x[n](|x[n]|)^{m-1} \cdot h_{m}[n],
$$

in which only the odd-order terms correspond to nonzero energy in the frequency band of interest, as revealed in [11]. Evidently, the first term is the linear component corresponding to the majority of the SI power, which can be estimated and cancelled using the algorithm proposed in (5). Furthermore, it was found in [11] that in practice the higher order terms of (6) constitute a correspondingly lower power, because those terms are created by the mixing of multiple lower-order terms, where each mixing operation reduces the combined power. Hence only a limited number of terms have to be considered in implementing practical SI leakage-channel estimation.

4) Open Research Issues in Digital Cancellation:

Once the SI signal can be sufficiently suppressed by performing both RF/analog and digital cancellation, the SI-contaminated signals can be successfully detected by the FD receiver. In contrast to analog cancellation, which is unable to adaptively combat the time-variant effects of the radio environment, digital cancellation is capable of dynamically adapting to these, because it estimates the SI leakage-channel on a short-term per-packet basis. Hence robust digital cancellation provides the necessary residual SI power reduction below the noise floor. It is shown in [11] that in a typical indoor deployment operating in the $2.4 \mathrm{GHz}$ Industrial Scientific Medical (ISM) band over a $80 \mathrm{MHz}$ bandwidth, a hybrid FD system is capable of reducing both the distortions as well as the effects of environmental changes by up to $110 \mathrm{~dB}$ in a dense indoor office environment. Furthermore, a median throughput gain of $87 \%$ can be achieved by practical WiFi radios, corresponding to almost twice the throughput of the conventional HD system. Nonetheless, a range of practical issues still have to be addressed, including:

- The Tradeoff between Analog and Digital Cancellations: Despite of the capability in cancelling the linear/non-linear SI components in digital cancella- tion, it is still noteworthy that digital cancellation operating in isolation without analog cancellation fails to provide a sufficiently high SI suppression [8]. To offer a high enough SI cancellation, a hybrid analog-digital design is required to successfully subtract all the linear and non-linear components. However, there exists a fundamental tradeoff between the capacities of analog- and digital-domain techniques. To elaborate a little further, the achievable SI-rejection of digital cancellation depends on the efficiency of the attainable suppression of the preceding analog cancellation when they are cascaded, with the capability of the former typically decreasing as that of the latter increases. In a scenario, where analog cancellation might achieve a sufficiently high suppression, digital cancellation may even become unnecessary. It would be very important to effectively balance the roles of the analog- and digital-domain functions in the overall cancellation and to carefully reveal the overall benefits of combined analog/digital cancellation.

- Performance Limitations Imposed by Practical Imperfections: Despite the promising capabilities offered by concatenated analog-digital cancellations, the joint benefits of analog-plus-digital cancellation may still remain limited by various practical factors, such as the phase noise issues elaborated on in Section III-E and the leakage-channel estimation errors. Specifically, the amount of attainable combined analog and concatenated digital suppression is limited by the phase noise imposed by the local oscillators [29]. If the analog canceller achieves a higher suppression, the residual SI has a dominant phase-noise-induced contribution. Otherwise the residual SI exhibits a higher correlation with the SI signal and thus the SI may be efficiently reduced by the digital canceller. Therefore, it is rather critical to substantially improve the aggregated performance of concatenated analog-digital cancellation by carefully combating the impact of practical distortions.

In summary, to carefully tackle the above-mentioned challenges and improve the aggregated capability of the concatenated analog-digital cancellation, we have to address several critical problems. On one hand, we must effectively balance the tradeoff between analog and digital cancellations so as to attain the best possible aggregated cancellation in the overall solution. On the other hand, the cancellation capability of any individual technique (e.g. passive suppression, analog or digital cancellation) should be further improved. To achieve 
this ambitious goal, we may resort to sophisticated algorithms, such as the SI model relying on Taylor series expansion [11] for mitigating both linear and non-linear SI components in the analog- and/or digital-domain. Furthermore, in contrast to the existing FD solutions relying on a single antenna [11] or a pair of antennas constituted by a TA and RA [10], the spatial diversity gain offered by multiple antennas can also be readily exploited for further improving the SI suppression capability, as elaborated on in the following subsection.

\section{MIMO Aided SI Cancellation Techniques by Exploit- ing the Spatial Diversity Gain}

The exploration of SISO based FD techniques motivated the development of SI mitigation techniques conceived for MIMO devices [81]. Three main approaches have been proposed, namely natural isolation (i.e. constituted by spatially separating the transmit and receive antenna arrays) [12], time-domain (TD) cancellation [10] and spatial-domain (SD) suppression [72], [74], [82], all of which are applicable to FD MIMO devices for improving the SI mitigation capability [19].

1) Natural Isolation: Unlike passive suppression techniques discussed in Section III-A, in which the SI strength is attenuated relying on path-loss effect, natural isolation in MIMO based FD systems can be achieved by employing some signal processing techniques (e.g. beamforming) to provide an additional man-made isolation [19]. For example, with separated receive and transmit antenna arrays, a rational antenna-array installation can guarantee obstacles in between the array to block the line-of-sight (LOS) SI component. Furthermore, directional antenna elements can be separated by pointing at opposite directions [61], thus substantially reducing the SI strength imposed on RAs.

2) Time-Domain Cancellation: Classic TD training can be beneficially utilized for estimating the leakage path of the SI and thus for facilitating a reliable cancellation, provided that the FD system knows its own transmitted signal (or at least approximately) [15], [19], [71]. If the SI channel can be reliably estimated, the SI signal may be effectively replicated and removed from the received signal. Time-orthogonal training based algorithms [15], [83] can be employed for satisfying the above-mentioned requirement. In contrast to methods using time-orthogonal training, the structured SI cancellation technique of [71] allows for the FD device to estimate the TA-RA leakage path of the SI by observing its own transmit signal during instances, when the source node is inactive. Hence, the FD device becomes capable of suppressing any SI imposed on the signal transmitted from the source node to it after estimating the TA-RA SI leakage, provided that the FD device's decoder has a priori knowledge of the SI. It was shown in [71] that the structured cancellation technique is capable of outperforming the time-orthogonal training based schemes of in terms of the achievable rate.

Despite of that, the main drawback of TD cancellation may be reflected in its blindness to the SD characteristics of the SI channel, such as a low-rank channel matrix that is not expected in creating a high isolation. Furthermore, the TD based schemes are sensitive to both the channel estimation errors and the transmit signal noise, corresponding to a new signal imposed on the FD input that may actually degrade the natural isolation as well as SI cancellation performance. The above-mentioned challenges may be addressed in spatial suppression techniques by employing the SD based techniques, which are found to be better than the TD based cancellation whenever a sufficient number of antennas compared to that of spatial streams is provided in the former [19]. Diverse SD solutions, such as precoding/decoding [9] and pre-nulling [55] have been proposed for rejecting the SI based on the idealized simplifying assumption of having perfect SI channel information.

3) Spatial Suppression: Spatial suppression can be achieved by employing schemes such as antenna subset selection, null-space projection (i.e. making the receiving and transmitting subspaces orthogonal by relying on precoding/beamforming techniques), and joint transmit and receive beam selection, which is capable of supporting more spatial streams by choosing the minimum eigenmodes for overlapping subspaces. Furthermore, algorithms such as zero-forcing (ZF) and MMSE can be employed for maintaining the desired signal quality as well as improving SI cancellations [19]. Instead of providing an exhaustive survey, we just introduce a number of typical schemes below.

- Antenna Subset Selection [84]: As revealed in [85], the simplified receive antenna selection scheme may inspire us to formulate SI suppression relying on generalized antenna subset selection using respective receive and transmit filters. In theory, the optimal filters can be found by calculating the Frobenius norm for all the TA-RA subset combinations and choosing the one corresponding to the lowest residual SI strength. However, deriving the optimal solution will become infeasible as the number of TAs and/or RAs becomes large. Instead, we may refer to some sub-optimal technique for facilitating a cost-efficient spatial suppression. In [21], [84], the so-called transmit antenna selection (TAS) scheme 
was proposed for achieving a high diversity gain at a low complexity. The main philosophy of TAS is that instead of the best subset antennas, a sub-optimal sub-set is selected for relay-aided transmission. As a benefit, the dimension of the SI leakage-channel matrix is reduced to $\left(N_{R} \times N_{k}\right)$, where $N_{R}$ and $N_{k}$ denote the number of RAs and the number of selected TAs, respectively. Consequently, the goal of TAS becomes to choose the specific subset of TAs that minimizes the effective SNR of the SI leakage-channel [21], with the following four criteria beneficially employed:

- Minimum Frobenius Norm SNR [21], which chooses the specific SI channel of the smallest Frobenius Norm from all the candidate selections;

- Minimum Post-Processing SNR [21], which chooses the subset of TAs with the minimum post-processing SNR for the sake of optimizing the BER performance;

- Minimum Singular Value [21], which chooses the specific antennas having the minimum singular values among all the SI channels;

- Minimum Capacity [21], which chooses the lowest-capacity SI leakage-channel after selecting the TA subset.

- Null-Space Projection [68]: In [68], a technique referred to as "Null-Space Projection" is proposed, in which the pre-coding and decoding matrices obtained from the singular value decomposition (SVD) of the SI channel are selected for ensuring that the FD device is capable of receiving and transmitting in different subspaces, i.e. the transmit beam-patterns are projected to the null-space of the SI channel combined with the receive filter and vice versa. The ZF filter provides an efficient solution, if the SI is dominant [68]. Furthermore, three filterdesign paradigms, i.e. independent design, which allows one of the filters to be designed without any knowledge of the other filter, separate design, which allows one of the filters to be designed given the other design, and joint design, in which both filters are designed together, can be implemented. If the pre-coding and decoding filters are jointly designed, several spatial input and output streams can be supported simultaneously by the FD device for outperforming the HD systems in terms of capacity [86].

- Pre-coding and Decoding [87]: If the transmit and receive filters can be jointly designed in a MIMO based FD device, an SI cancellation technique can be conceived by satisfying the condition of $\boldsymbol{D}_{R}^{H} \boldsymbol{H}_{R} \boldsymbol{P}_{R}=\mathbf{0}$, where $\boldsymbol{P}_{R}$ and $\boldsymbol{D}_{R}$ describe the pre-coder's and the decoder's action, respectively. Note that the pre-coding problem at hand corresponds to converting a MIMO channel matrix into matrices having a lower triangular structure (e.g. for AF relaying) or a diagonal matrix (e.g. for DF relaying) [87]. By invoking the SVD of $\boldsymbol{H}_{R}$ as $\boldsymbol{H}_{R}=\boldsymbol{U}_{R} \boldsymbol{\Sigma}_{R} \boldsymbol{V}_{R}^{H}$, efficient SI cancellation can be achieved, provided that the precoder and decoder matrices are designed as $\boldsymbol{P}_{R}=$ $\alpha\left[\boldsymbol{v}_{R, j \neq k} \cdots \boldsymbol{v}_{R, j \neq k}\right]$ and $\boldsymbol{D}_{R}=\left[\boldsymbol{u}_{R, k} \cdots \boldsymbol{u}_{R, k}\right]$, with $\boldsymbol{u}_{R, k}$ and $\boldsymbol{v}_{R, k}$ representing the k-th column vector of the matrices $U_{R}$ and $V_{R}$, respectively, and with $\alpha$ denoting the power normalization factor.

- Optimal Eigenbeamforming [72]: In a MIMO based FD device, SD aided SI suppression can be performed using optimal eigenbeamforming, which minimizes the power of the residual SI signal by pointing the transmit and receive beams to the minimum eigenmodes of the SI channel [72]. The optimal eigenbeamforming is capable of increasing both the attainable communication rate and the coverage area [88]. Apart from frequency-domain beamforming, a TD transmit beamforming (TDTB) method may also be invoked at the RF front-end of the receivers in broadband FD mode MIMO radios. A cancellation capability of $50 \mathrm{~dB}$ is achievable in TDTB over a reasonably wide bandwidth of $30 \mathrm{MHz}$ [16]. Furthermore, distributed beamforming can also be employed for multiuser MIMOassisted FD systems in order to suppress the SI at the FD device (e.g. base station) as well as to mitigate the multiuser interference at the mobile stations [89].

- SI Suppression with Maximum SIR [90]: In this scheme, the FD device employs both transmit and receive filters for suppressing the SI signal, hence maximizing the ratio between the power of the useful signal and the SI power [90]. The SIR may be readily maximized either at the input or the output of the device. By defining the receive and transmit suppression matrices as $\boldsymbol{G}_{r x}$ and $\boldsymbol{G}_{t x}$, respectively, a two-step approach can be employed for optimizing the SIR:

- Step 1: By designing the receive suppression matrix $\boldsymbol{G}_{r x}$ and neglecting for the moment the transmit suppression matrix $\boldsymbol{G}_{t x}$, the specific $\boldsymbol{G}_{r x}$ matrix that is optimal in terms of SIR maximization at the FD device's receiver has 
to obey [90] $\boldsymbol{G}_{r x, o p t}=\arg \max _{\boldsymbol{G}_{r x}} \frac{\left\|\boldsymbol{G}_{r x} \boldsymbol{H}_{1}\right\|_{F}^{2}}{\left\|\boldsymbol{G}_{r x} \boldsymbol{H}_{0}\right\|_{F}^{2}}$, where $\|\boldsymbol{A}\|_{F}^{2}$ denotes the Frobenius norm of the matrix $\boldsymbol{A}$, while the matrices $\boldsymbol{H}_{0}$ and $\boldsymbol{H}_{1}$ represent the SI leakage-channel matrix and the device's receive channel matrix, respectively.

- Step 2: Given $\boldsymbol{G}_{r x}, \boldsymbol{G}_{t x}$ is optimized by maximizing the SIR at the FD device's transmit side, yielding $\boldsymbol{G}_{t x \text {,opt }}=$ $\arg \max _{\boldsymbol{G}_{t x}} \frac{\left\|\boldsymbol{H}_{2} \boldsymbol{G}_{t x}\right\|_{F}^{2}}{\left\|\boldsymbol{G}_{r x, o p t} \boldsymbol{H}_{0} \boldsymbol{G}_{t x}\right\|_{F}^{2}}$, where $\boldsymbol{H}_{2}$ represents the device's transmit channel matrix [90]. SI suppression with maximum SIR was shown to outperform the ZF nulling technique in terms of its channel capacity, regardless whether the SI channel is of full-rank or not [90]. Furthermore, enhanced joint-nulling schemes may also be conceived for MIMOaided FD devices for mitigating the SI and for maximizing the ergodic rate simultaneously [74].

- MMSE Filtering [68]: The degree of freedom (DoF) in the spatial domain allows for more sophisticated approaches to be conceived for improving the useful signal power. Suffice to say that the family of SD suppression schemes is capable of minimizing the effects of SI at the cost of partially affecting the useful signal. An MMSE cost-function based scheme can be developed for reducing the aforementioned cost. This is, because in contrast to ZF, the MMSE scheme can suppress not only the SI signal, but also the additive Gaussian noise [68], hence typically leading to a better BER than ZF. Furthermore, a multiple-filter based scheme can also be developed for achieving a substantial inter-antenna/multistream interference suppression as well as SI cancellation [91]. The total mean squared error of the signal received at the mobile stations can thus be minimized by using this filter. As compared to the conventional scheme [92], which designs the interantenna/multi-stream interference suppression and SI cancellation independently, the joint multiplefilter based scheme is capable of achieving a superior performance in terms of the average BER.

From the above-mentioned discussions, while both TD and FD techniques having their respective advantages, each technique has exposed its drawbacks. For example, TD cancellation suffers from a higher residual SI induced by the channel estimation errors and transmit signal noise, while SD suppression requires extra antennas and hence suffers from a high computational complexity. To elaborate a little further, many of the existing SD suppression methods rely on complex matrix computations, which may significantly erode the benefits of FD techniques owing to their cost [82]. Carefully designed lowcomplexity algorithms conceived for high-dimensional MIMO SI channels are thus capable of dramatically reducing the hardware/software cost in FD signal processing, hence facilitating realistic FD communication. Furthermore, the combination of TD cancellation and SD suppression may be potentially capable of providing a high isolation level for a moderate number of antennas even at a low SNR and may perform better than any of these techniques operating in isolation [19]. In [19], the combination of TD and SD cancellations is found to be especially beneficial if there exists a significant amount of transmit signal noise. Combining these schemes results in a residual SI leakage-channel expressed in the form of $\hat{\boldsymbol{H}}_{R}=\boldsymbol{G}_{r x} \boldsymbol{H}_{R} \boldsymbol{G}_{t x}+\boldsymbol{C}$, with $\boldsymbol{G}_{r x} \boldsymbol{H}_{R} \boldsymbol{G}_{t x}$ representing the SD suppression and $\boldsymbol{C}$ denoting the TD cancellation. The corresponding filter design can thus be performed for one of the schemes first, followed by the other scheme based on the residual SI leakage-channel of the first scheme [68].

\section{E. IMPAIRMENTS IMPOSED ON SELF- INTERFERENCE CANCELLATION}

Despite the successful implementations of FD techniques in the context of short-range communications, extending their applications to the long-range communication scenarios remains a challenge, again, owing to the impact of SI [29]. As indicated in [4], the attainable aggregate SI cancellation does not increase linearly with the achievable analog cancellation. Furthermore, the amount of attainable digital cancellation depends on the amount of the analog cancellation, when they are cascaded. In other words, when the analog cancellation reduces the SI to a lesser extent, the digital canceller becomes capable of mitigating more substantially and vice versa. More specifically, the reasons of "what limits the amount of analog cancellation in a FD system and how does the amount of cancellations by analog and digital cancellers depend on each other in cascaded systems" are still unclear [29].

To find the reasons for the above-mentioned limitations, a number of factors, including the transmitter/receiver phase noise [29], I/Q imbalance [76], power amplifier nonlinearity [76] and ADC quantization noise [66], etc., all of which impose impairments on the TARA link of FD systems, must be taken into consideration [29]. Furthermore, we may resort to the following methods for mitigating the above-mentioned impairments: 
- The quantization noise level can be reduced below the thermal noise level relying on a high-resolution ADC [12]. Hence the FD radio is capable of striking off the quantization noise from the list of SIcancellation bottlenecks. For instance, as indicated in [66], an ADC with 16 bit quantization should be exploited in order to handle SIR in the range of $-40 \mathrm{~dB}$ to $-20 \mathrm{~dB}$ relying on digital cancellation;

- The I/Q imbalance can be readily mitigated as long as it does not significantly vary with time [29]. This distortion as well as the power amplifier's nonlinearity can be compensated with an aid of digital pre-distortion in the transmitter [93];

- If the FD model operates in the linear region of the power amplifier, the SI cancellation will no longer suffer significantly from the power amplifier's nonlinearity [29].

Given the above-mentioned prerequisite, the phase noise [94] inflicted by the jitter in the local oscillators, turns out to constitute a major bottleneck in FD systems, since it substantially limits the amount of SI cancellation. However, depending on the specific system parameters, such as the power amplifier linearity, ADC resolution, precision of the RF/analog cancellation and the SI leakage-channel estimation accuracy, the major factors leading to performance limitations may vary substantially. As revealed in [76], provided that the effects of quantization/phase noise can be mitigated, the nonlinearity of the power amplifier and the transmit I/Q imbalance will dominate the precision of digital cancellation, if the most dominant leakage-channel component's SI contribution can be subtracted from the SI signal during the RF/analog cancellation phase. To elaborate a little further, let us consider a system with a transmit power of $10 \mathrm{dBm}$ and a bandwidth of $20 \mathrm{MHz}$ (corresponding to a noise floor of $-101 \mathrm{dBm}$ ). In this scenario the radio is required to suppress the effects of both quantization/phase noise and sampling jitter by about $40 \mathrm{~dB}$ to mitigate the SI below the noise floor, whereas the distortions induced by the power amplifier nonlinearity and transmit I/Q imbalance must be suppressed by about $80 \mathrm{~dB}$ [76].

In the following, we will analyze the impact of the above-mentioned impairments on SI cancellation in consideration of variant scenarios.

1) The Impact of Phase Noise on the Self-Interference Cancellation: In this section, the impact of phase noise on the SI cancellation is analyzed, with all the abovementioned impairments (including I/Q imbalance, power amplifier nonlinearity, etc) but channel estimation errors assumed to be mitigated. In order to model the phase noise imposed on the SI signal, let us first consider the baseband signal $x(t)$ that is up-converted to a carrier frequency of $\omega_{c}$. The up-converted signal can be expressed as:

$$
x_{\text {up }}(t)=x(t) e^{\jmath\left[\omega_{c}+\phi(t)\right]},
$$

where $\phi(t)$ represents the phase noise. The downconverted phase noise can be similarly defined. The variance and autocorrelation of the phase noise can thus be defined as $\sigma_{\phi}^{2}=\mathbb{E}\left\{|\phi(t)|^{2}\right\}$ and $R_{\phi}(\tau)=$ $\mathbb{E}\{\phi(t) \phi(t-\tau)\}$, respectively.

As quantified in [29], the strength of the residual SI can be represented as

$$
\mathbb{E}\left\{\left|y_{\text {residual }, d}[i T]\right|^{2}\right\}=\left|h_{S I}\right|^{2} \sigma_{\phi}^{2}\left(1-R_{\phi}(d T)\right)+2 \sigma_{\text {noise }}^{2},
$$

where $y_{\text {residual }, d}[i T]$ denotes the residual SI for a given delay $d$ between the SI and the SI-inverse-based cancelling reference signals, $h_{S I}$ stands for the attenuation of the SI signal, $T$ represents the sampling period and $\sigma_{\text {noise }}^{2}$ is the variance of the thermal noise. Once the delay $d$ is sufficiently high, the residual SI power will depend only on the variances of the phase noise and of the thermal noise.

In order to evaluate the impact of phase noise on the SI cancellation, let us define the phase noise and its corresponding variance in the SI signal as well as the cancelling signal by the pairs $\left(\phi_{\mathrm{si}}(t), \sigma_{\mathrm{si}}^{2}\right)$ and $\left(\phi_{\text {cancel }}(t), \sigma_{\text {cancel }}^{2}\right)$, respectively. Furthermore, let us denote the phase noise at the receiver and its variance by the pair $\left[\phi_{\text {down }}(t), \sigma_{\text {down }}^{2}\right]$. Without loss of generality, we assume that $\phi_{\mathrm{si}}(t)$ and $\phi_{\text {cancel }}(t)$ are independent of $\phi_{\text {down }}(t)$. As compared to the family of narrowband systems, a wideband system suffers from a relatively high thermal noise level, but the phase noise floor remains similar [29].

- The impact of phase noise on analog cancellation: In [29], the authors analyzed the impact of phase noise on the residual SI strength. Considering an imperfect SI leakage-channel estimation in the analog domain, the strength of the residual SI in each canceller stage (including the pre-mixer, post-mixer and baseband canceller) comprises two components, namely the SI-dependent component and the phase noise-dependent component. As indicated in [29], the SI-dependent component can be further reduced by employing a digital canceller. The phase-noise-dependent residual SI, on the other hand, scales linearly in strength with the SI power, implying that the phase noise will dominate the residual SI after analog cancellation in the presence of a high received SI power level. Both the 
theoretical analysis and the experimental results of [29] showed that the amount of analog cancellation attained is inversely proportional to the phase noise variance. Specifically, the amount of pre-mixer cancellation is limited by the inverse of the phase noise variance, but the amount of cancellation can be increased by carefully matching the local oscillators to the SI and cancelling signals. Unlike the premixer cancellers, in which the delay determines the amount of residual SI, the post-mixer cancellers are capable of reducing the residual SI by improving the SI channel estimation accuracy. In baseband analog cancellers, on the other hand, the residual SI is found to be proportional to the sum of the phase noise variances at both the TA and RA.

- The impact of phase noise on the relationship between the passive suppression and the analog cancellation: In [29], the authors evaluated the relationship between the passive suppression and the analog cancellation under the impact of phase noise, showing that a higher cascaded passive suppression and analog cancellation can be provided by increasing the passive suppression capability. Specifically, the total cancellation can be maximized, if the passive suppression is maximized. However, the aggregate SI cancellation does not linearly increase with the attainable passive suppression, because the amount of analog cancellation in a pre-mixer canceller is dependent on the amount of passive suppression. Explicitly, the former reduces, as the latter increases.

- The impact of phase noise on the cascaded SI cancellation: In a cascaded SI cancellation scheme, both analog and digital cancellations will suffer from a performance degradation induced by the effects of phase noise [29]. When analog cancellation is performed in conjunction with idealized perfect SI leakage-channel estimation, the digital cancellation fails to further reduce the residual SI, when any one of the following conditions is met:

- $\phi_{\mathrm{si}}(t)$ and $\phi_{\text {cancel }}(t)$ are identically distributed in the pre-/post-mixer cancellers, and $\phi_{\mathrm{si}}(t)$ and $\phi_{\text {down }}(t)$ are identically distributed in the baseband analog cancellers;

- $\phi_{\mathrm{si}}(t)$ and $\phi_{\text {cancel }}(t)$ are not independently distributed, and $\left\{\begin{array}{l}\sigma_{\text {si }}^{2} \ll 1 \\ \sigma_{\text {cancel }}^{2} \ll 1 \\ \sigma_{\text {down }}^{2} \ll 1\end{array}\right.$ is satisfied;

If, on the other hand, realistic imperfect SI leakagechannel estimation is considered in the context of analog cancellation, the cascaded digital cancel- lation becomes capable of subtracting (only) the residual SI component, provided that it is correlated with the SI signal. However, the total amount of the cascaded cancellations still remains limited by both the phase noise properties and the errors of the SI leakage-channel estimation executed within the analog cancellation stage. Specifically, a poor analog cancellation leads to degraded overall cancellation, even if digital cancellation benefits for idealized perfect SI leakage-channel estimation.

2) The Impact of Power Amplifier Nonlinearity and of Transmit I/Q Imbalance on the Digital Cancellation: As revealed in [29], [76], a number of impairments such as quantization/phase noise and power amplifier nonlinearity will impose a limit on the attainable grade of SI cancellation. In order to combat the above-mentioned impairments, the authors of [76] focused their attention on the design of digital cancellation by decomposing the SI signal into its non-delayed contribution and the nonline-of-sight (NLOS) components. They used digitaldomain cancellation to supplement the RF/analog cancellation. After cancelling the LOS component relying on RF techniques, the quantization/phase noise (due to the impact of RF/analog impairments) can be reduced to a level that no longer limits the performance of the FD system. Instead, the nonlinearity of the power amplifier and the transmit I/Q imbalance become the most prominent factors ${ }^{12}$ limiting the precision of SI cancellation, in which case the NLOS components should be eliminated by using a digital cancellation scheme. Taking the abovementioned impairments into consideration, the following algorithms can be employed [76]:

- The power-amplifier-output based algorithm of [76] can be invoked by using the output signal of the power amplifier, which can be obtained by attaching a coupler to the TA. The coupled signal will then be converted to its digital samples by using another RF front-end. In this case the SI channel can be estimated using the classical LS algorithm of [11].The numerical results of [76] show that the SI components caused both by the power amplifier nonlinearity and by the transmit I/Q imbalance can be substantially reduced, thus achieving a higher digital cancellation capability.

- The two-stage iterative algorithm of [76] employed for mitigating the limitations of the SI

\footnotetext{
${ }^{12}$ In consideration of the fact that the effects of quantization error, phase noise and sampling jitter can be made low enough by adjusting the TA-RA isolation, the impairments associated with the power amplifier nonlinearity and transmit I/Q imbalance will then dominate the distortion of digital cancellation [76].
} 
leakage-channel estimation. Since the SI leakagechannel estimation process is contaminated by interference (in this scenario the interference is contributed by the desired signal), the SI cannot be completely eliminated. Although in theory the leakage-channel estimation accuracy can be improved by increasing the training sequence length, the time-variant channel variation (when the channel can no longer be treated as static one within a longer training sequence period) will erode the accuracy of leakage-channel estimation. In order to address the above-mentioned issues, the far-end (desired) signal can be removed from the aggregate received signal for the sake of improving the SI leakage-channel estimation accuracy and hence the attainable SI cancellation. In the first stage of this algorithm, the SI cancellation can be carried out by implementing classic channel estimators, such as the LS algorithm of [11] and the decision-feedback algorithm [95], followed by detecting and regenerating the desired transmit signal in the second stage. Following the current-round of SI cancellation, the far-end signal can be detected again, followed by employing this re-detected signal in a new iteration of SI leakage-channel estimation and cancellation. The above-mentioned procedure can be repeated, until a satisfactory cancellation is achieved, resulting in the output SINR level required.

As compared to the power-amplifier-output based algorithm, the two-stage iterative algorithm is capable of substantially suppressing the impairments imposed by the NLOS components. However, the detection errors of the desired signal in the current iteration will impact the SI leakage-channel estimation in the next iteration, resulting in a phenomenon known as "error propagation". To improve the system's resilience to error propagation, the technique of soft decision [95] instead of hard decisions is preferred. However, the hard decision can still be employed for effectively improving the system's performance, as long as the initial detection error is moderate [76].

\section{F. Comparison of Variant SI Suppression Techniques}

Although numerous sophisticated techniques have been proposed for SI cancellation in FD radios, there are advantages and disadvantages in the context of each approach. For instance, antenna-aided cancellation constitutes a promising active SI suppression technique, which has an up to $60 \mathrm{~dB}$ cancellation capability in combination with both noise reduction and digital cancellation, but some basic limitations are also observed, such as a radically degraded performance upon increasing the bandwidth, a stringently specified inter-antenna distance, etc [7]. Furthermore, despite of promising cancellation capability provided by existing solutions (e.g. up to $110 \mathrm{~dB}$ cancellation capability offered by the circulator-aided cancellation scheme [11]), the complexity-capability tradeoff must be addressed. For example, the delay-line based cancellation circuit complicated the hardware design, and most of the SD suppression methods suffer from a higher computational complexity than their TD cancellation based counterpart owing to the requirement of complex matrix computations in the former. More importantly, in practical systems, the hardware imperfections, such as the non-flat frequency response of analog technique, the phase noise, power amplifier nonlinearity, and I/Q imbalance, etc. will all impose constraints on the SI cancellation. Finally, the family of passive suppression techniques, on the other hand, can provide a substantial SI rejection by exploiting the associated path-loss, but may impose physical constraints on the size of the devices. In summary, each mechanism has its own advantages and disadvantages, as briefly summarized in Table VI. To sufficiently exploit the (theoretically) promising FD benefits, we may resort to the properly designed MAC/higher-layer protocols for making up for the shortcomings of the physicallayer techniques as well as effectively mitigating the performance degradations due to the problems such as long end-to-end delay, high PLR, and hidden terminal distortions.

\section{MAC-LAYER PROTOCOL DESIGN FOR FULL-DUPLEX SYSTEMS}

Although the sophistically designed SI cancellation methods executed in the physical layer are capable of providing a promising performance gain in terms of throughput, the most interesting FD benefits may occur at the higher-layer protocols such as MAC-layer protocols [10], [15], which may be capable of mitigating the end-to-end delay, the network congestion and the hidden terminal problems [7]. For instance, a bottleneck may occur at the Wireless Local Area Networks (WLAN) AP, which serves multiple clients with the same channel access opportunity as a client. The abovementioned bottleneck can be removed by employing an FD mode AP, which is capable of transmitting whenever clients send packets to it. Furthermore, in CSMA/CA protocol, the hidden terminal problem, which occurs due to the fact that the HD mode receiver cannot inform the other nodes of an ongoing reception, is naturally mitigated in FD systems due to the receiver's capability 
TABLE VI

AdVantages and DisadVantages of Variant SupPression Mechanisms

\begin{tabular}{|c|c|c|c|}
\hline Category & Algorithm & Advantages & Disadvantages \\
\hline $\begin{array}{l}\text { Passive } \\
\text { Suppression }\end{array}$ & $\begin{array}{l}\text { Directional } \\
\text { Diversity [13]; } \\
\text { Antenna } \\
\text { Separation [12]; } \\
\text { Antenna } \\
\text { Cancellation [7] }\end{array}$ & $\begin{array}{l}\text { 1) Attenuates SI power by } \\
\text { taking advantage of path-loss } \\
\text { effect or SI nulling; } \\
\text { 2) Alleviates the SI cancellation } \\
\text { burden at the following active } \\
\text { cancellation stages }\end{array}$ & $\begin{array}{l}\text { 1) Passive SI suppression highly } \\
\text { depends on antenna separations } \\
\text { and configurations; } \\
\text { 2) Constrained by various } \\
\text { factors such as device size }\end{array}$ \\
\hline \multirow[t]{2}{*}{$\begin{array}{l}\text { Active } \\
\text { Suppression }\end{array}$} & $\begin{array}{l}\text { Analog } \\
\text { Cancellation } \\
{[7],[10]} \\
{[15],[63]}\end{array}$ & $\begin{array}{l}\text { 1) SI being suppressed } \\
\text { prior to the receiver RF front-end; } \\
\text { 2) Quantization noise at the input of } \\
\text { the ADC can be reduced; } \\
\text { 3) Substantially extracts SI at RA } \\
\text { relying on techniques such as } \\
\text { SI invert circuit }\end{array}$ & $\begin{array}{l}\text { 1) Hardware imperfections constrained } \\
\text { 2) Phase noise/other impairments } \\
\text { limit the cancellation capability; } \\
\text { 3) Performance degradation in wideband } \\
\text { due to non-flat frequency response } \\
\text { 3) The analog-only cancellation } \\
\text { is insufficient for decoding }\end{array}$ \\
\hline & $\begin{array}{l}\text { Digital } \\
\text { Cancellation } \\
{[11],[76]}\end{array}$ & $\begin{array}{l}\text { 1) Further reduces the residual SI } \\
\text { after analog cancellation; } \\
\text { 2) Dynamic adaptation of distortions } \\
\text { on a per-packet basis }\end{array}$ & $\begin{array}{l}\text { 1) Digital cancellation alone cannot } \\
\text { provide high enough SINR for decoding; } \\
\text { 2) Power amplifier nonlinearity } \\
\text { and I/Q imbalance constrained; } \\
\text { 3) Performance degraded if preceded } \\
\text { by a powerful analog canceller }\end{array}$ \\
\hline
\end{tabular}

of immediately starting a transmission (i.e. this operation basically suppresses the nearby nodes' transmissions) [10]. In summary, it may open up the possibility of boosting the overall throughput of FD based wireless networks by employing new MAC-layer protocols [93].

However, designing an efficient MAC-layer protocol for FD systems would be a rather challenging task [7], because it requires the FD device to be capable of reliably receiving a packet from a node, while transmitting to another node. Furthermore, hidden terminals may significantly degrade the FD mode transmissions and receptions. Considering the hidden terminal topology shown in Fig. 6(a), where Node 2 is beyond the reliable detection radio range of Node 1 . Assuming that the AP has a packet for Node 1 and Node 2 has a packet for the $\mathrm{AP}$, the key question arises, as to whether the FD mode can be enabled in an asynchronous manner in order to admit a new traffic flow. There are two potential actions for the FD AP:

- AP starts a new reception session, while transmitting: Let us assume that the AP is transmitting to Node 1, when Node 2 initiates the transmission of a packet. Then the AP has to estimate the channel between Node 2 and itself so as to decode Node 2 's packet. "Dirty" estimation ${ }^{13}$ is required in the asynchronous FD mode, with either the BER or channel capacity, or in fact both degraded. Explicitly, the BER associated with dirty estimation may become a factor six higher compared to that of clean estimation [15]).

- AP starts a new transmission session, while receiving: When the AP has already commenced

\footnotetext{
${ }^{13}$ In [15], the physical layer channel estimation in the presence of ongoing transmission is labelled as "dirty" estimation.
}

receiving a packet from Node 2 and intends to send a packet to Node 1 in order to exploit its FD capability, this mode must not be activated, because the high-power uncancelled SI will completely saturate the receiver's AGC, hence potentially contaminating the ongoing reception.

In brief, the asynchronous FD mode potentially facilitates reception while transmitting, albeit at some performance loss. In order to address the above-mentioned challenges, new MAC protocols specifically for FD mode systems must be implemented. As indicated in [15], one of the fundamental principles for designing a FD-based MAC protocol is to provide opportunities for all nodes to access the wireless channel, while trying to maximize the overall network throughput as well as maintaining fairness to all users. The key challenge is to reliably find the opportunities to transmit and receive simultaneously in a completely distributed manner, while substantially suppressing the hidden terminal collisions. To satisfy the above-mentioned requirements, several high-efficiency MAC-layer protocols have been proposed [10], [15].

\section{A. The Busytone-aided MAC Protocol [10]}

Recalling from Section I-A that the FD gains brought about by the physical-layer techniques cannot be fully exploited, unless a carefully designed FD-based MAClayer protocol is developed for supporting these physicallayer advances [10]. For instance, the attainable FD gains may be eroded by the so-called hidden terminals. Let us consider a simple case, a pair of FD nodes exchange their packets, while Node 1 and Node 2 cannot directly hear from each other, as illustrated in Fig. 6 (a). A primary transmitter (i.e. Node 1 in this example) 


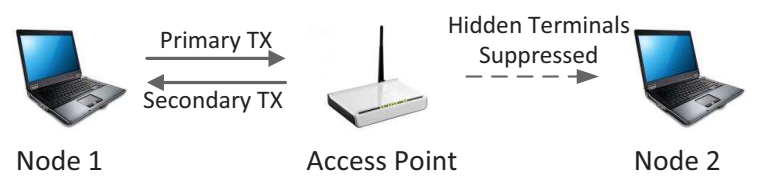

(a) Full duplex with hidden terminals

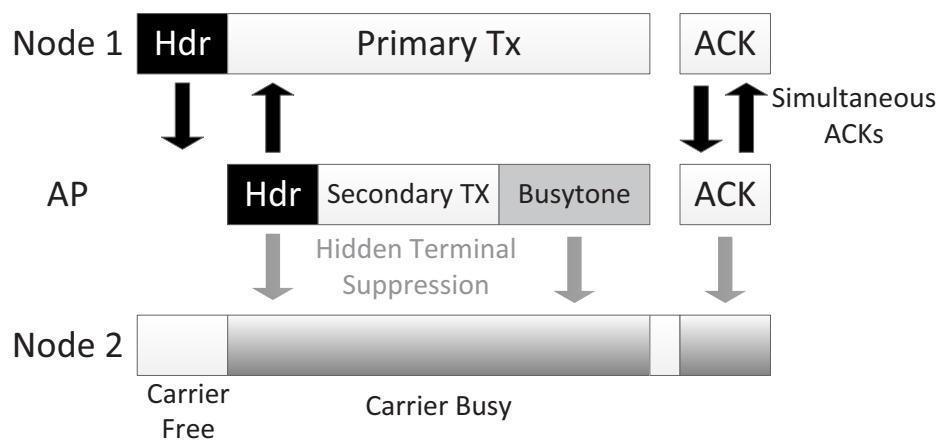

(b) Full duplex packet exchange

Fig. 6. The FD MAC protocol protects the primary and secondary transmissions from hidden terminal losses, in which a busytone field is introduced in the data frame to protect the periods of single-ended data transfer (Fig. 9 of [10]).

initiates its transmission using the standard CSMA/CA protocol, while the primary receiver (i.e. the AP) initiates a secondary transmission, once it detects the header of the primary transmission. Bearing in mind that the primary and secondary packets are offset in time and may even have different lengths, the AP's secondary transmission to Node 1 may be completed before Node 1 's primary transmission to the AP, hence causing a collision by Node 2's transmission if at that moment only Node 1 transmits.

In order to mitigate the above-mentioned hiddenterminal problem, a modified MAC protocol employing the busytone field is proposed in [10]: Whenever a node completes its transmission before finishing its reception, it must transmit a predefined signal, until its reception ends, thus effectively circumventing the hidden terminals' collision. If by any chance a secondary node has no secondary packet to send, when it receives a primary transmission, the busytone should be sent immediately after it decodes the header of the primary packet.

The above-mentioned protocol was implemented in Rice University's WARP V2 platform [96], which uses a WiFi-like packet format and 64-subcarrier OFDM physical-layer signaling in a $10 \mathrm{MHz}$ bandwidth. It was shown experimentally [10] that at the data rate of $2 \mathrm{Mb} /$ sthe proposed busytone aided MAC protocol is capable of preventing $88 \%$ of the collision-induced packet losses whilst maintaining a packet reception ratio of $83.4 \%$, hence far exceeding that attained by the HD protocols (i.e. 52.7\%). Naturally, the FD protocol is unable to perfectly prevent the hidden terminal based collisions, because the secondary transmissions start only after the primary header's reception. A higher packet loss ratio is imposed by the increased collisions for a higher traffic-load condition. When the tele-traffic load increased to $4 \mathrm{Mb} / \mathrm{s}$, the packet reception ratio of the proposed FD based MAC protocol was reduced to $68.3 \%$.

\section{B. FD-MAC Protocol [15]}

Although the above-mentioned busytone aided FD MAC protocol may effectively mitigate the hidden terminal collisions, the transmissions of busytone field inevitably erode the wireless resource utilization. Another MAC protocol referred to as FD-MAC [15] is capable of combating the hidden terminal problem in infrastructure-based WiFi-like networks without relying on the busytone-padding cost. Three mechanisms, including Shared Random Backoff (SRB), Snooping and Virtual Contention Resolution, are implemented in FDMAC protocol, as illustrated in Fig. 7.

- Shared Random Backoff (SRB) [15]: This mechanism temporarily couples the backoff counter of a pair of nodes, which have discovered that they have a packet destined for each other. Once a pair of nodes discover that they have more packets 


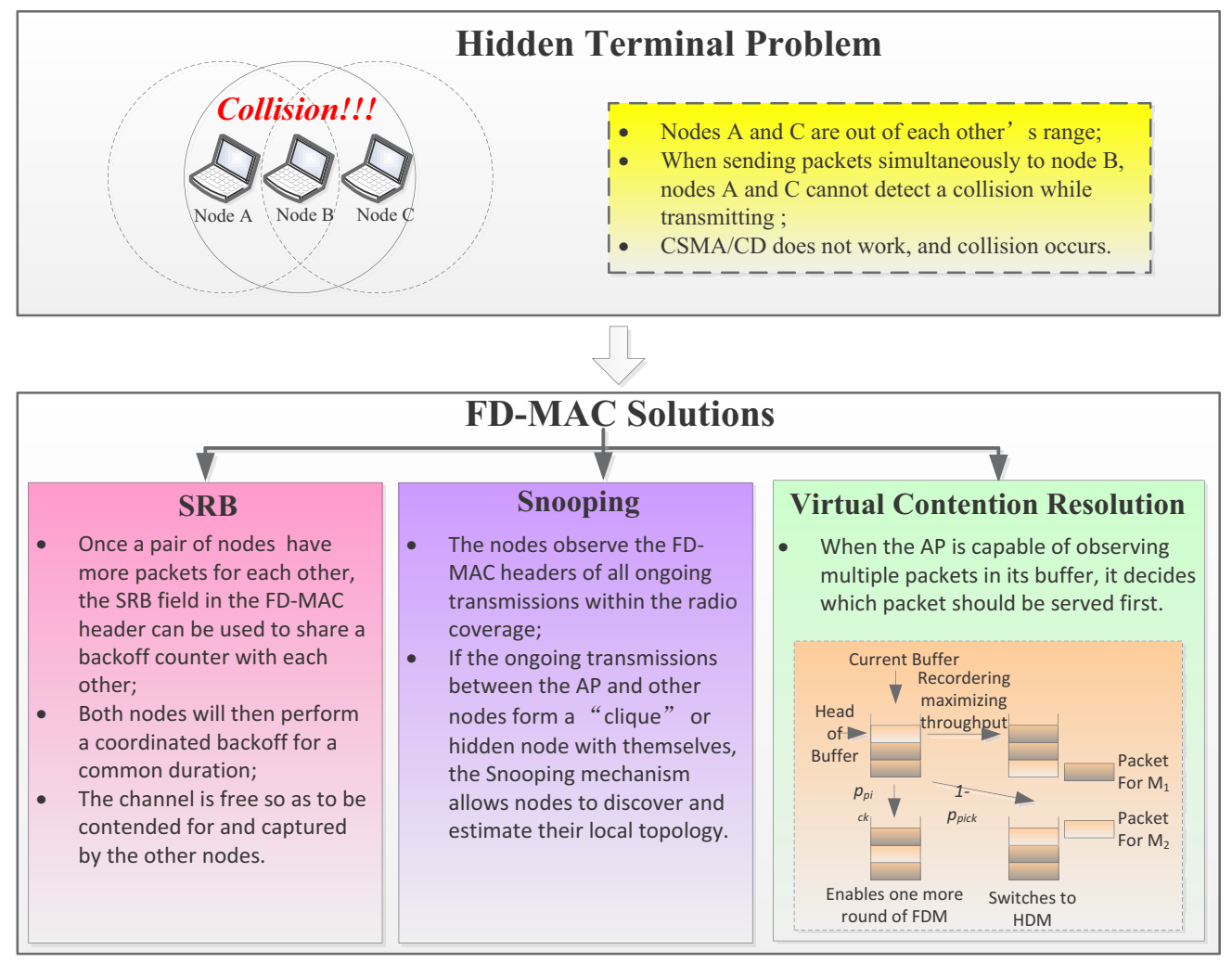

Fig. 7. Mechanisms of FD-MAC protocol [15], including Shared Random Backoff (SRB), Snooping and Virtual Contention Resolution, can be employed for addressing the problem of hidden terminal (Fig. 2 of [6]).

for each other, the 10-bit SRB field found in the FD header of each packet will be used by these two nodes to share a backoff counter with each other. Both nodes will then perform a coordinated backoff for a common duration in order to stay synchronized, whilst at the same time freeing the channel, which hence can be temporarily contended for and captured by the other nodes.

- Snooping [15]: Even when the nodes have frozen their counters, this "snooping" mechanism still requires the nodes to observe the headers of all ongoing transmissions within the radio coverage area. To elaborate a little further, the packet snooping mechanism allows nodes to estimate their local topology and to discover, if the ongoing transmissions between the AP and the other nodes form a "clique" or hidden node with themselves. The node is unable to exploit FD operation, when aiming for discovering a clique in order to prevent its new transmissions from colliding with the ongoing traffic flow.

- Virtual Contention Resolution [15]: When the AP is capable of observing multiple packets in its buffer, it statistically decides, which of the packets it will serve first. The AP may also opt for using FD mode by inspecting multiple packets in the queue. In order to reduce the probability of an AP delaying the transmission of its head of line (HOL) packets, which would impose problems on the higher-layer protocols, FD-MAC proposes to occasionally send a non-HOL packet, albeit only with a vanishingly low probability.

Seamless wireless access can be guaranteed by the FD-MAC protocol, whilst maximizing the benefits of the FD capability. Experimental results have shown that FDMAC achieves a throughput gain of up to $70 \%$ over its comparable HD counterpart [15].

\section{Open Research Issues in Full-Duplex MAC-Layer Protocols}

As described in this section, some critical problems widely happened in wireless networks, such as packet loss events imposed both by collisions and by the hidden terminal phenomenon, can be effectively addressed by 
the FD mode systems relying on appropriately designed MAC protocols [15]. Despite of that, numerous open challenges still have to be resolved, such as the compatibility with the existing HD protocols, design of lowpower MAC protocols and the conception of FD MAC protocols for cognitive radio networks (CRNs). In the following, a number of challenges encountered during the design of FD MAC protocols will be discussed, accompanied by a range of potential research directions.

1) Compatibility with Existing HD MAC Protocols: The FD MAC protocol must be backwards compatible with the existing HD MAC protocols [15]. Specifically, when implementing the hidden-terminal-mitigation protocols, the secondary transmitter has to identify the mode of the primary node (i.e. whether the primary is FD capable), otherwise they may suffer from poor interactions in terms of link-layer establishment [10]. Furthermore, the access mechanism should not unduely favor FD opportunities over HD flows, so as to provide a fair opportunity for all nodes to access the shared medium. In addition, in a CR environment, the MAC protocol has to enable an FD node to identify the other primary/cognitive nodes that are capable of engaging in FD communications. The above-mentioned requirement is especially critical for exploiting the FD capability in hybrid systems supporting both HD and FD devices [15].

2) Quick Response in Real-Time Protocols: Implementing a real-time FD-based MAC protocol requires low-latency of hardware response from the FD devices. For example, in the Busytone-aided MAC Protocol of [10], a primary receiver remains susceptible to collisions, until it completes the reception of the primary transmission's packet header, which is equivalent to a period of $56 \mu \mathrm{s}$ in the IEEE 802.11a standard [10]. Furthermore, in CRNs, although FD techniques are promising in terms of improving the primary receiver's detection, finding optimal decision processes may not be feasible in highmobility environments, because the convergence speed of FD based MAC operations must be substantially improved in order to facilitate prompt spectrum-allocation decisions in the face of high-velocity mobility [15].

3) The Increased Memory Requirements [10]: To reduce the PLR of the FD mode, a sufficiently large buffer is required for avoiding the queue overflow. For example, in order to combat the collisions due to hidden terminals, the FD-based MAC protocols, such as that proposed in [10], may require an FD mode node to pre-load multiple packets in response to its peer's transmission, thus demanding more memory than HD mode.

4) Effectively Handling Asymmetric Traffic [10]: While the FD MAC protocol (e.g. busytone-padding protocol [10]) strives for mitigating the hidden terminal collisions in asymmetric-traffic scenarios, it unavoidably degrades the resource exploitation due to the busytone transmission. When multiple short packets have to be transmitted by an FD node in response to a long received packet, efficiently utilizing rather than wasting the secondary channel (by busytones) still constitutes an open challenge.

5) Reducing the Energy Consumption of Full-Duplex $M A C$ Protocols [15]: The energy dissipation of FD MAC protocols remains a challenging issue, because most wireless terminals are battery-driven and have limited energy harvesting capabilities. Apart from the lowpower consideration in designing cancellation circuit, it is also of great importance to develop cost-efficient FD MAC protocols having a low energy consumption, which would extend the devices' battery recharge-time as well as the overall network's survivability.

\section{IMPLEMENTATION, ENHANCEMENT AND OPTIMIZATION ISSUES IN FD SYSTEMS}

FD mode is shown to be more spectrally efficient than HD mode, because only one channel is need per two hops in the former [19]. Recently, the FD systems relying on efficient SI cancellation techniques have attracted a considerable attention both in academia [97] and in industry [23], [24]. Furthermore, several prototypes of FD transceivers employing variant SI cancellation techniques have been built for demonstrating the feasibility of FD communication, showing the substantial performance gains over HD mode [7], [10], [12], [98]. However, the existing framework maximizes the attainable system throughput either without imposing any delay constraint [7], [10] or under a stringent delay constraint [12], [98]. In practice, these two extreme remains may not adequately characterize the users' requirements, who tend to have diverse delay constraints. Therefore, refined algorithms should be proposed for practical FD systems for mitigating the above-mentioned deficiencies.

In this section, a range of critical issues related to the implementation, performance enhancement and optimization of the FD systems will be discussed, including the design of hybrid HD/FD relaying, optimal relay selection and optimal power allocation. For the readers' convenience, we have summarized the major contributions on the implementation and optimization issues in Table VII.

\section{A. Hybrid HD/FD Relaying}

As evaluated by [10], FD mode and conventional HD mode (e.g. HD mode MIMO) offer their respective advantages under different scenarios, i.e. robustness 
TABLE VII

MAJOR TECHNiQues Associated TO IMPLEMENTATION AND OPTIMIZATION ISSUES

\begin{tabular}{|c|c|c|c|c|}
\hline Year & Author(s) & Contribution & Complexity & Assumption \\
\hline \multirow[t]{2}{*}{2006} & $\begin{array}{l}\text { Mesbah et al. } \\
\text { [99] }\end{array}$ & $\begin{array}{l}\text { Derives a closed-form expression for the } \\
\text { optimal power allocation for an achiev- } \\
\text { able rate region of FD cooperative mul- } \\
\text { tiple access systems. }\end{array}$ & Low & $\begin{array}{l}\text { Channel State Information is available at } \\
\text { the transmitters. }\end{array}$ \\
\hline & $\begin{array}{l}\text { Shibuya et al. } \\
{[100]}\end{array}$ & $\begin{array}{l}\text { Deploys FD relays with SFN-based } \\
\text { broadcast systems using OFDM. }\end{array}$ & Low & $\begin{array}{l}\text { The antenna array elements are separated } \\
\text { by no less than half a wavelength. }\end{array}$ \\
\hline \multirow[t]{2}{*}{2009} & $\begin{array}{l}\text { Riihonen et } \\
\text { al. [101] }\end{array}$ & $\begin{array}{l}\text { Controls the relay transmission power } \\
\text { according to the SI channel information } \\
\text { error as well as the other channel infor- } \\
\text { mation }\end{array}$ & Low & $\begin{array}{l}\text { Avoid the need for relay-destination } \\
\text { channel state information in the relay. }\end{array}$ \\
\hline & $\begin{array}{l}\text { Song et al. } \\
{[102]}\end{array}$ & $\begin{array}{l}\text { Studies power control for cooperative } \\
\text { cellular networks comprising multiple } \\
\text { sources, multiple FD AF relays, and a } \\
\text { destination. }\end{array}$ & Low & $\begin{array}{l}\text { Individual power constraints are assumed } \\
\text { at the sources. }\end{array}$ \\
\hline 2010 & $\mathrm{Rui}_{[103]}$ et al. & $\begin{array}{l}\text { Improves both the average capacity and } \\
\text { symbol error rate by employing FD relay } \\
\text { selection. }\end{array}$ & Low & $\begin{array}{l}\text { An i.i.d. Rayleigh fading environment is } \\
\text { assumed. }\end{array}$ \\
\hline \multirow[t]{4}{*}{2011} & $\begin{array}{l}\text { Yang et al. } \\
\text { [104] }\end{array}$ & $\begin{array}{l}\text { Derives the optimal powers of the sec- } \\
\text { ondary source and relay in CR networks } \\
\text { to minimize the outage probability of } \\
\text { these nodes. }\end{array}$ & Low & $\begin{array}{l}\text { Independent and non-identically dis- } \\
\text { tributed Nakagami- } m \text { fading channels } \\
\text { are assumed. }\end{array}$ \\
\hline & $\begin{array}{l}\text { Yamamoto et } \\
\text { al. }[105]\end{array}$ & $\begin{array}{l}\text { Utilizes the optimal transmission } \\
\text { scheduling approach in a hybrid HD/FD } \\
\text { scheme to improve the end-to-end } \\
\text { throughput. }\end{array}$ & Medium & Flat-fading channels are considered. \\
\hline & $\begin{array}{l}\text { Miyagoshi et } \\
\text { al. [106] }\end{array}$ & $\begin{array}{l}\text { Proposes a scheduling scheme in the } \\
\text { time duration for a hybrid HD/FD relay- } \\
\text { ing system comprising one source, one } \\
\text { relay and two destinations. }\end{array}$ & High & $\begin{array}{l}\text { The optimization problem is formulated } \\
\text { as a non-linear programming problem. }\end{array}$ \\
\hline & $\begin{array}{l}\text { Riihonen et } \\
\text { al. [22] }\end{array}$ & $\begin{array}{l}\text { Employs the combination of opportunis- } \\
\text { tic relaying mode selection and transmit } \\
\text { power adaption to maximize the instan- } \\
\text { taneous and average spectral efficiency. }\end{array}$ & Medium & $\begin{array}{l}\text { Both the instantaneous and average spec- } \\
\text { tral efficiencies are attainable. }\end{array}$ \\
\hline \multirow[t]{2}{*}{2012} & $\begin{array}{l}\text { Krikidis et al. } \\
\text { [107] }\end{array}$ & $\begin{array}{l}\text { Proposes AF scheme for FD relay se- } \\
\text { lection with multiple relays to combine } \\
\text { the spatial diversity benefits with high } \\
\text { spectral efficiency. }\end{array}$ & High & $\begin{array}{l}\text { Different instantaneous information is } \\
\text { assumed to be available. }\end{array}$ \\
\hline & $\begin{array}{l}\text { Cheng et al. } \\
\text { [26] }\end{array}$ & $\begin{array}{l}\text { Proposes optimal resource allocation } \\
\text { schemes for wireless HD/FD relay net- } \\
\text { works for support the statistical QoS } \\
\text { provisioning. }\end{array}$ & Low & $\begin{array}{l}\text { Diverse QoS requirements over flat- } \\
\text { fading wireless relay networks are con- } \\
\text { sidered. }\end{array}$ \\
\hline \multirow[t]{3}{*}{2013} & $\begin{array}{l}\text { Zhong et al. } \\
\text { [108] }\end{array}$ & $\begin{array}{l}\text { Proposes a new DF protocols for FD } \\
\text { relay selection and derives the closed- } \\
\text { form expressions for both the channel } \\
\text { capacity and OP. }\end{array}$ & Low & $\begin{array}{l}\text { The i.i.d. Rayleigh fading channels are } \\
\text { assumed. }\end{array}$ \\
\hline & Lee ${ }_{\text {[109] }}^{\text {et }}$ & $\begin{array}{l}\text { Realizes the FD relay in multiuser } \\
\text { MIMO relaying systems by employing } \\
\text { a distributed beamforming scheme. }\end{array}$ & High & $\begin{array}{l}\text { Channel reciprocity is assumed to be } \\
\text { exploited. }\end{array}$ \\
\hline & $\begin{array}{l}\text { Zheng et al. } \\
\text { [110] }\end{array}$ & $\begin{array}{l}\text { Studies the achievable region of CRNs } \\
\text { using FD cognitive base station. }\end{array}$ & High & $\begin{array}{l}\text { The primary system always tries to op- } \\
\text { erate in its full power. }\end{array}$ \\
\hline
\end{tabular}

at low SNR (e.g. lower than $12 \mathrm{~dB}$ in experiment of [10]) using HD mode MIMO to sufficiently exploit the diversity gains, and high throughput with FD mode under high SNR environment (e.g. higher than $12 \mathrm{~dB}$ [10]) as long as the SNR loss due to the residual SI remains below $1.5 \mathrm{~dB}$. Therefore, a hybrid scheme, which adopts the benefits of both modes depending on the instantaneous channel conditions and the availability of CSI at the transmitter, can be implemented to enable switching between HD mode and FD mode and may be expected to outperform any individual mode over the entire SNR range. Several techniques for implementing hybrid schemes have been proposed, such as
1) Opportunistic Hybrid Scheme [22]: Opportunistic duplex-mode resource allocation is motivated by the fundamental tradeoff between the achievable spectral efficiency and the SI suppression attained. In [22], explicit conditions are provided under which a specific duplex mode is preferred over the other, with the benefit of opportunistic switching between the two modes evaluated. The opportunistic "hybrid FD/HD relaying" was shown [22] to be capable of offering significant performance gains over the conventional system design that is confined to either mode. Furthermore, the tradeoff between the FD mode and HD mode is heavily dependent on the employment of transmit power adaptation, with a 
potential to make FD modes more attractive. It is shown in [22] that both the FD mode and hybrid HD/FD mode are attractive techniques in terms of achieving a higher spectral efficiency than the HD mode in infrastructurebased relay-aided links.

2) Resource Scheduling for Hybrid Schemes [106]: In cellular radio networks the base station experiencing a high traffic load may take advantage of FD operation in order to fully exploit its capacity. HD mode, on the other hand, imposes a lower cost and thus can be performed by the mobile terminals. In practical systems, as to whether the FD mode is preferred or not may be decided depending on the specific value of a control parameter referred to as the cancellation coefficient ${ }^{14}$. If the cancellation coefficient is higher than a pre-defined threshold $k_{t h}$, FD mode should be used for achieving a higher effective capacity, otherwise, it is desirable to use HD mode. Dynamic hybrid resource allocation policies can thus be developed for both the FD and HD modes for maximizing the network's throughput under diverse QoS requirements. However, the careful coordination of HD and FD operations constitutes a challenge [32], [111]. Specifically, as found in [32], the HD operation tends to reduce the user-fairness, potentially leading to an unstable operating point in terms of resource scheduling, when more and more terminals have to be admitted. To address the above-mentioned issues, specific resource allocation/scheduling mechanisms can be implemented for facilitating an effective hybrid $\mathrm{HD} / \mathrm{FD}$ mode to satisfy diverse QoS requirements, such as

- TD Scheduling for Five-Phase based Hybrid of Full- and Half-duplex Relaying [106]: A TD scheduling scheme is proposed in [106] for enabling a hybrid of full- and half-duplex relaying (FHDR). This scheme extends a simple single-user hybrid FHDR to a system comprising a source, a relay and two destinations, as illustrated in Fig. 8. Five orthogonal TD phases are considered, with the first three phases corresponding to the HD mode, whilst the fourth and fifth phases corresponding to the FD mode. Proportional fairness in terms of user's end-to-end throughput [112] can be achieved by using the hybrid FHDR. As compared to an equalopportunity scheduling scheme, where an identical transmit time is allocated for each user, the hybrid FHDR of [106] is capable of achieving a superior performance in terms of the sum-rate without jeopardizing the fairness among the users [106].

- SI Cancellation Coefficient aided Resource Al-

\footnotetext{
${ }^{14}$ Cancellation coefficient was introduced in [26] to characterize the effect of SI on FD mode.
}

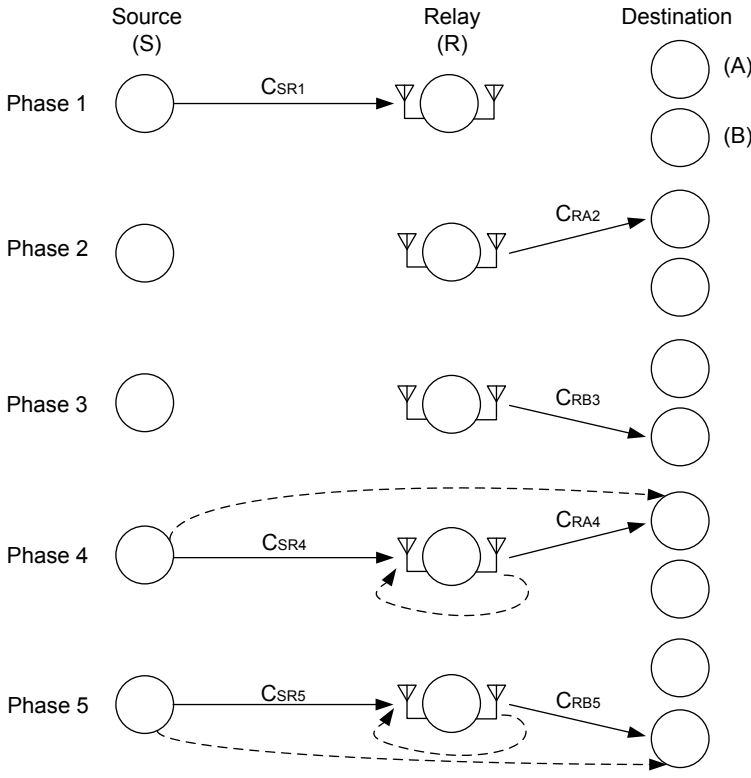

Fig. 8. Scheduling scheme with 5 phases in the time duration for a hybrid of full- and half-duplex relaying (Fig. 1 of [106]).

location for Hybrid HD/FD mode [26]: Optimal resource allocation schemes can be proposed [26] for wireless HD/FD networks in order to support the required quality of service (QoS), which is achieved by integrating information theory with the principle of "effective capacity". As indicated in [26], the effective capacity of perfect FD mode relying on optimal resource allocation is simply twice as high as that of HD mode. Furthermore, it has been shown in [26] that the hybrid HD/FD transmission mode is capable of achieving a better performance than using any of these two stand-alone modes.

3) Hybrid Schemes in Cognitive Radio Networks [110], [113]: To improve the spectrum utilization, a CR paradigm can be employed by enabling active cooperation between the primary and cognitive systems [56]. As indicated in [15], FD techniques are promising in terms of both extending the achievable data rate region and addressing the problem of primary receiver detection in CRNs by facilitating a prompt spectrumallocation decision. By enabling FD mode in the cognitive base station or relay, it is capable of relaying the primary signal while transmitting its own cognitive signal on the same channel simultaneously. Furthermore, the achievable primary-cognitive rate region for the proposed system can be found by addressing the cognitiverate-maximization problem with either optimal or suboptimal solution [110]. 
- Optimal Solution: In [110], FD based cooperative CR technique is propose, in which the cooperation can be performed in two phases: During Phase $\mathrm{I}$, the primary user transmits and the FD mode base station listens, while in Phase II, the FD mode base station can both forward the primary signal and transmit its own cognitive signal. In consideration of the fact that the FD mode may not always outperform the HD mode, a hybrid scheme that switches between the two modes can be implemented for providing extra performance gain. In theory, when we perform the mode selection, we can simply solve the maximum-rate problem for each mode and choose the better one. However, it would be very complicated to derive the closedform representation of each maximization problem, let alone give insights on which mode is preferred under various conditions.

- Sub-Optimal Solution: In order to address the above-mentioned challenge, the authors in [110] proposed a sub-optimal solution based on the classic ZF criterion [113] (to null out the interference between the primary and secondary systems) for performing mode selection. Considering the cognitive BS equipped with 6 antennas, when it works in the FD mode, approximately 50\% higher rates for both the primary and cognitive users can be achieved, compared with the HD mode with the same RF chains (i.e. 4 TAs and 2 RAs). Furthermore, the proposed hybrid scheme is capable of performing nearly as well as the best mode selection. Specifically, the hybrid HD/FD scheme has been shown [110] to be capable of achieving almost three times the cognitive user rates provided by the HD mode with the aid of the same RF chains. Additionally, if the cognitive base station operates in the AF mode with a transmission power of $25 \mathrm{dBm}$, the hybrid scheme may achieve an OP of below $40 \%$, which is much lower than that of HD mode (estimated to be $60 \%$ in [110]). A lower OP of $32 \%$ can even be achieved by using the DF relaying mode with a transmission power of $20 \mathrm{dBm}$ or higher [110].

\section{B. FD Relay Selection for Cooperative Communications Systems}

In cooperative communication systems comprising multiple relays, relay selection has been widely considered in HD based relaying mode as a benefit of its high performance in terms of capacity/OP of wireless links, efficient exploitation of the system resources (e.g. power and bandwidth), and hardware simplicity [114]-[116]. These advantages accrue from the fact that the system typically selects relays receiving halfway between the source and destination and that having more relays tends to lead to an improved performance, because the system combines the independently fading signals of several relays [107]. However, the relay selection policies of FD relaying have not been explored in sufficient depth, hence their impact on the system performance is virtually unknown. Therefore below we touch upon relay selection techniques conceived for FD protocols.

1) Relay Selection for AF protocol [36]: In [117], relay selection procedures are designed for FD based AF cooperative communication systems. The simplified system model considers a single source-destination pair and multiple relays, with the direct source $\rightarrow$ destination link assumed to suffer from a strong attenuation. Hence, communication can only be established via the cooperative relays. In each time slot, a single relay is selected, which employs a cancellation scheme for reducing the impact of SI, to assist the source's transmission [36]. In [36], an optimal relay selection procedure maximizing the instantaneous FD channel capacity is considered in addition to several reduced-complexity sub-optimal relay selection policies that rely on partial CSI knowledge for relaying channel. Furthermore, an optimal relay selection technique, which incorporates a hybrid relaying strategy that dynamically switches between the FD and HD modes according to the near-instantaneous residual SI has also been investigated [107], showing that the hybrid scheme is capable of outperforming both the HD and FD relaying modes.

2) Relay Selection for DF protocol: In this section, we will briefly consider the performance of FD based DF protocols [108]. A cooperative relaying network comprising a source node $S, N$ FD DF relays denoted by the set of $\Omega=\left\{R_{i}, i=1,2, \ldots N\right\}$, and a destination $D$, is considered. Similarly, the direct link is also assumed to suffer from deep fading, hence $S$ can only communicate with $D$ via $R_{i}$. We also assume that $S$ and $R_{i}$ transmit at a power of $P_{S}$ and $P_{R}$, respectively. All wireless links in the cooperative network suffer from additive noise with zero mean and $N_{0}$ variance.

By using the opportunistic DF relay selection model, the most beneficial relay can be chosen according to

$$
k=\arg \max _{i: R_{i} \in \Omega} \min \left\{\gamma_{R_{i}}, \gamma_{D}\right\}
$$

where $\gamma_{R_{i}}$ and $\gamma_{D}$ denote the received SNR of the $i$ th relay $\left(R_{i}\right)$ and $D$, respectively. For a pre-set SNR 
threshold $\gamma_{t h}{ }^{15}$, the OP of the opportunistic relay selection scheme can be expressed as [108]

$P_{\text {out }}=\left\{1-\frac{P_{S} \bar{\gamma}_{S R} \cdot \exp \left[-\left(\frac{1}{P_{R} \bar{\gamma}_{R D}}+\frac{1}{P_{S} \bar{\gamma}_{S R}}\right) \cdot \gamma_{t h}\right]}{P_{S} \bar{\gamma}_{S R}+P_{R} \bar{\gamma}_{L I} \cdot \gamma_{t h}}\right\}$

where $\gamma_{L I}$ denotes the residual SI-to-noise ratio.

The capacity of opportunistic DF relay selection may be formulated as

$$
C=\alpha \cdot \beta \cdot \mathrm{E}\left\{\log _{2}\left(1+\gamma_{e q}\right)\right\},
$$

where $\alpha=\left\{\begin{array}{l}1, \quad \text { FD Mode } \\ 1 / 2, \quad \text { HD Mode }\end{array}\right.$ denotes the duplexing factor, and $\beta$ represents the signal bandwidth. The optimal power allocation can thus be carried out in the following two cases.

- Optimum Power Allocation (OPA) Relying on Individual Power Constraints (IPC): In the presence of SI signal, the optimal power allocation (OPA) is critical for optimizing the performance of FD communication networks. By and large, power allocation techniques in communication systems can be classified into two modes, finally those relying on the individual power constrains (IPC) and on the sum power constrains (SPC) [118]. In the former, the individual power of both the source and relay should be decided by the control unit. In the latter, on the other hand, only the sum power of the source and relay is considered. The corresponding power allocation problem can be formulated as

$$
\begin{aligned}
& \left(P_{S}^{*}, P_{R}^{*}\right)=\arg \max _{\left\{P_{S}, P_{R}\right\}} C, \\
& \quad \text { subject to } 0 \leq\left\{P_{S}, P_{R}\right\} \leq 1,
\end{aligned}
$$

which leads to [108]

$\begin{cases}\bar{P}_{R}^{*}=1, \quad \text { if } \bar{\gamma}_{L I}>\frac{\bar{\gamma}_{R D}}{4\left(\bar{\gamma}_{S R}-\bar{\gamma}_{R D)}\right)} \& \bar{\gamma}_{S R}>\bar{\gamma}_{R D}, \\ \bar{P}_{R}^{*}=\frac{-\bar{\gamma}_{R D}+\sqrt{\bar{\gamma}_{R D}^{2}+4 \bar{\gamma}_{L I} \bar{\gamma}_{S R} \bar{\gamma}_{R D}},}{2 \bar{\gamma}_{L I} \bar{\gamma}_{R D}}, \text { otherwise. }\end{cases}$

- OPA Relying on Sum-Power Constraints (SPC): The power allocation problem is formulated as

$$
\begin{aligned}
\left(P_{S}^{*}, P_{R}^{*}\right) & =\arg \max _{\left\{P_{S}, P_{R}\right\}} C, \\
& \text { subject to } P_{S}+P_{R}=2 \text { and } 0 \leq\left\{P_{S}, P_{R}\right.
\end{aligned}
$$

\footnotetext{
${ }^{15}$ The threshold $\gamma_{t h}$ is related to the target rate $R$. When the cooperative networks work in FD mode, we have $\gamma_{t h}=2^{R}-1$. By contrast, in HD mode, $\gamma_{t h}=2^{2 R}-1$ is assumed.
}

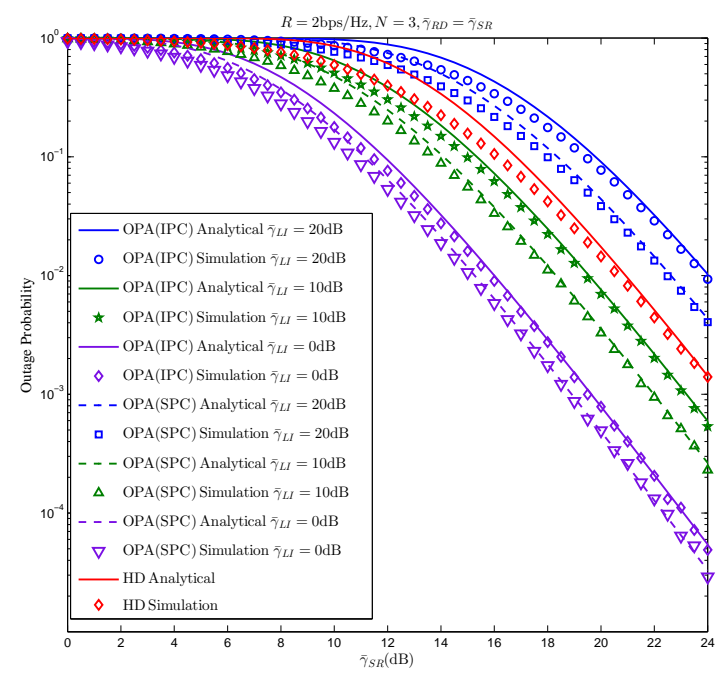

Fig. 9. Performance comparison between half- and FD (considering OPA under IPC and SPC) modes in terms of OP for different values of $\bar{\gamma}_{L I}$.

which leads to

$$
\left\{\begin{array}{l}
\bar{P}_{R}^{*}=\frac{-\left(\bar{\gamma}_{R D}+\bar{\gamma}_{S R}\right)+\sqrt{\left(\bar{\gamma}_{R D}+\bar{\gamma}_{S R}\right)^{2}+8 \bar{\gamma}_{S R} \bar{\gamma}_{R D} \bar{\gamma}_{L I}}}{2 \bar{\gamma}_{R D} \bar{\gamma}_{L I}}, \\
\bar{P}_{S}^{*}=2-\bar{P}_{R}^{*} .
\end{array}\right.
$$

Fig. 9 compares the performance of both HD and FD modes in terms of the OP for a data rate of $R=2 \mathrm{bps} / \mathrm{Hz}$ and $\bar{\gamma}_{L I}=10 \mathrm{~dB}$. Observation in the figure shows that FD mode outperforms HD mode, and OPA under SPC performs better than that under IPC. Furthermore, the performance comparisons between HD and FD modes in terms of their average capacity are shown in Fig. 10 and Fig. 11. By keeping $N=3$ unchanged, FD mode is shown to outperform HD mode in terms of its average channel capacity, if we have $\bar{\gamma}_{L I} \leq 0 \mathrm{~dB}$. Additionally, when $\bar{\gamma}_{L I}=0 \mathrm{~dB}$ and $\bar{\gamma}_{S R}=\bar{\gamma}_{R D} \in[0 \mathrm{~dB}, 40 \mathrm{~dB}]$, the FD mode is capable of achieving a throughput advantage over the HD mode, which is in the range of $33.1 \% \sim 87.6 \%$. Finally, by keeping $\bar{\gamma}_{L I}=5 \mathrm{~dB}$ unchanged, Fig. 11 shows that the average channel capacity improvement of FD mode is more substantial in the highSNR region. For instance, when $\bar{\gamma}_{S R}=\bar{\gamma}_{R D}=10 \mathrm{~dB}$, the FD mode outperforms the HD mode in terms of a $23.5 \% \sim 34.9 \%$ higher throughput, and this advantage

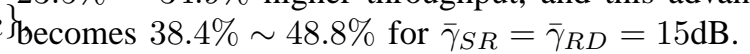

\section{Optimal Power Allocation [99], [102], [119]}

Power allocation may play an important role in fully exploiting the potential of FD techniques as well as 


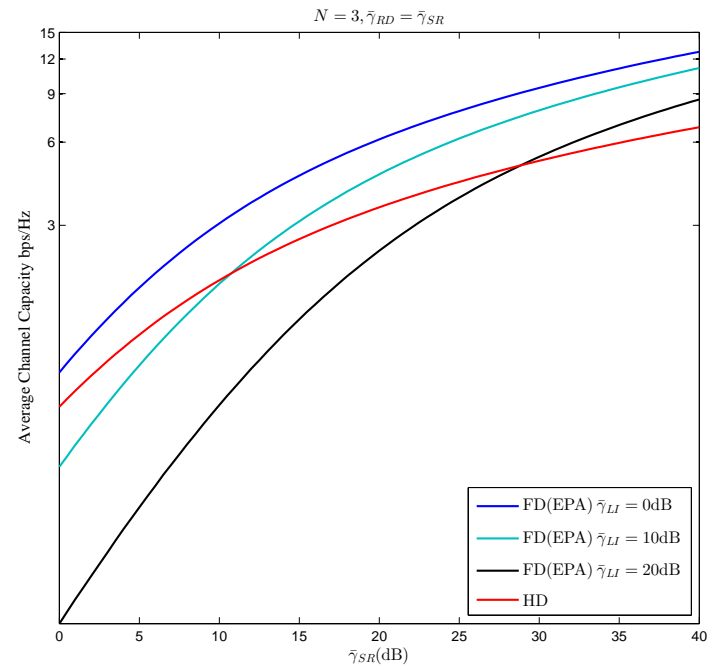

Fig. 10. Performance comparison between half- and FD modes in terms of average channel capacity for different values of $\bar{\gamma}_{L I}$.

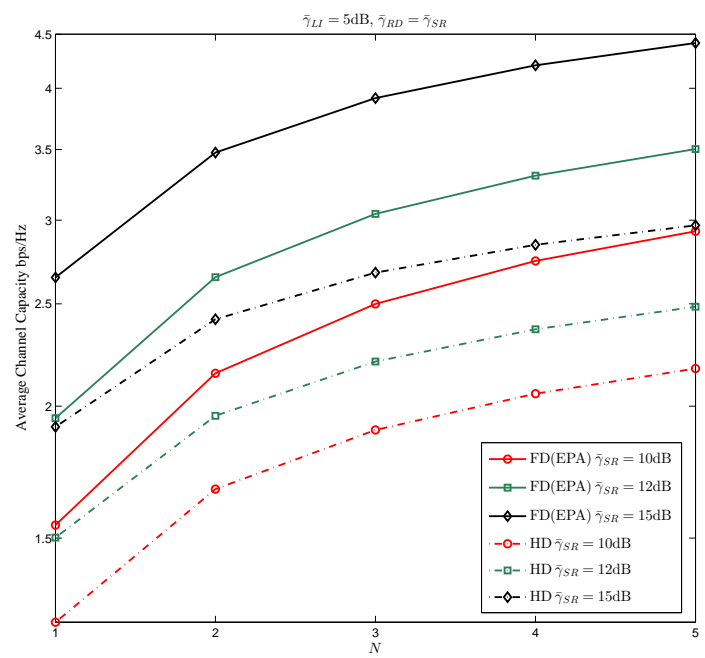

Fig. 11. Performance comparison between half- and FD modes in terms of average channel capacity for different values of $\bar{\gamma}_{S R}$. satisfying diverse QoS requirements by controlling the SI strength. Since a higher transmit power usually implies a stronger SI power imposed on FD RAs, the objective of power control is to assign the minimal transmission power for "just" satisfying the users' SINR requirements. One of the motivations for studying adaptive power allocation in FD devices arises from the system's potential instability imposed either due to the impact of SI or due to the similarity of the received and of the amplified transmit signal (e.g. in the AF relaying mode) [28]. There are numerous studies on developing distributed power control algorithms for FD systems [99], [102], [104], [119], [120], which are briefly reviewed below:

- In [99], an expression for characterizing the achievable rate-region of FD cooperative multiple access systems under optimal power allocation has been derived, where the natural non-convex formulation of the power allocation problem was transformed into a convex form, leading to a closed-form solution for a range of practical scenarios. Apart from that, power control designed for cooperative networks comprising multiple sources, multiple FD based AF relays, and a destination is also studied [102]. This algorithm updates each source's power by treating the other sources' signal as interference, until all the sources converge to a stable fixed-power mode of operation. Compared to the HD relays, the power controlled system relying on FD relays is capable of achieving substantial benefits.

- In FD based CR networks, the primary user experiences interference imposed both by the secondary source and by the relay due to the FD operation. It would thus be critical to optimize the transmission powers at both the secondary source and at the FD relay for the sake of improving the performance of all the users. When the total sum of transmission powers at both the secondary source and relay is constrained, the optimal power allocation can be derived by solving the equation [104]

$$
\begin{aligned}
\frac{g_{S R} P_{S}}{\sigma_{R}^{2}+a_{R R} P_{R}} & =\frac{g_{R D} P_{R}}{\sigma_{D}^{2}+a_{S D} P_{S}}, \\
\text { subject to } & b_{S P} P_{S}+b_{R P} P_{R} \leq I_{t h},
\end{aligned}
$$

where $P_{S}$ and $P_{R}$ represent the transmission powers of the secondary source and of the relay, respectively, $g_{S R}, g_{R D}$ and $a_{S D}$ denote the channel gains of the source $\rightarrow$ relay, relay $\rightarrow$ destination and source $\rightarrow$ destination links, respectively, $\sigma_{R}^{2}$ and $\sigma_{D}^{2}$ stand for the noise variances at the secondary relay and destination, respectively, while $a_{R R}$ represents the gain of the SI leakage channel at the relay, and finally $I_{t h}$ is a predetermined threshold. 
- In [119], an outage-constrained power allocation scheme is proposed for CRNs. Unlike [104], the knowledge of the instantaneous CSI for the link between the primary and secondary users is not required in this scheme. However, the secondary user is allowed for sharing the spectrum of the primary user, provided that the primary user's maximum tolerable OP is satisfied [120]. Numerical results showed that the proposed optimal power allocation schemes are robust to the effects of the outdated CSI and achieve performance advantages over the Equal Power Allocation (EPA) scheme in terms of the OP of the secondary user.

\section{CONCLUSIONS AND POTENTIAL FUTURE RESEARCH}

\section{A. Conclusions}

The available radio spectrum is limited, hence before new commercially implementable spectral resources are exploited, the ever increasing throughput requirements cannot be readily satisfied without increasing the achievable spectral efficiency expressed in bits/s/Hertz. The main driving force behind FD techniques is the promise of nearly doubling the data rate in comparison to their HD counterpart, while striking an attractive trade-off amongst the design challenges. Those challenges specifically pertain to FD communications, potentially facilitating simultaneous transmission and reception within the same frequency band. One of the most challenging factors is that the family of SI suppression/cancellation solutions is typically based on complex and/or costly hardware designs. Hence it is of crucial importance to closely examine cost-efficient algorithms associated with tolerable hardware/software complexity. More importantly, the most dominant hardware imperfections, such as the phase noise, non-flat frequency response of the circuits, power amplifier nonlinearity and transmit I/Q imbalance, etc. may all impose limitations on the attainable SI cancellation capability and must be carefully mitigated. Furthermore, the effect of the MAC and other higher-layer protocols on the practical implementation of FD systems constitutes another key issue to be more vigorously investigated. Against the aforementioned requirements and challenges, there is an urging demand for high-performance, low-complexity FD protocols. In this section, we offer a few general design guidelines for FD wireless communication systems based on the solutions discussed throughout this treatise.

1) FD techniques may significantly improve both the achievable spectral efficiency and the associated network throughput compared to the classic HD approach, provided that the SI encountered at the FD nodes can be significantly reduced. Since any individual SI component (either linear or nonlinear) of insufficiently low level may become the limiting factor of the ultimate SI cancellation and may hence dominate the FD-gain degradations, each component should be substantially reduced by employing an appropriate technique (e.g. analog and/or digital cancellation). Although employing costly high-precision hardware can lead to a high SI cancellation capability, impairments such as the non-flat frequency response of the hardware and the dispersion of the SI leakagechannel may still impose a performance limit on the attainable RF/analog cancellation. Therefore, the performance of SI cancellation techniques in practical systems has to be further improved with the aid of better SI-cancellation techniques conceived for a higher transmit power and for a wider bandwidth. Furthermore, the other impairments, including phase noise, power amplifier nonlinearity and transmit I/Q imbalance, will also substantially degrade the performance of digital cancellation. To sufficiently reduce both the linear and non-linear SI components, the constraints imposed by above-mentioned impairments also have to be mitigated.

2) Digital cancellation exploits the knowledge of the interfering signal in order to cancel it after the contaminated received signal has been quantized by an ADC, thus improving the jointly designed analogand-digital cancellation capability. It is noteworthy that the maximum attainable SI suppression of digital cancellation decreases as that of the preceding analog cancellation increases in a concatenated regime. Hence the fundamental tradeoff between the capacities of the analog- and digital-domain techniques have to be addressed in order to effectively balance their roles achieving the highest possible overall benefits for the combined scheme. However, again, the joint benefits of cascaded analog/digital cancellation may be constrained by the various sources of distortions, such as the phase noise, power amplifier nonlinearity and transmit I/Q imbalance. It was experimentally shown in [29] that the phase noise imposes a limit on the amount of cascaded analog and digital cancellation. Specifically, the phase noise will dominate the residual SI after performing analog cancellation, hence preventing the concatenated digital canceller from further reducing the residual SI 
(if it is uncorrelated with the SI signal). Furthermore, the change of system parameters, such as the power amplifier linearity characteristics, ADC resolution, precision of the RF/analog cancellation and the SI leakage-channel estimation accuracy, etc. may change the major factors that dominate the attainable performance. To further improve the aggregated performance of concatenated analogdigital cancellation in practical FD systems, it is rather critical to combat the above-mentioned impairments and to adaptively accommodate the time-variant environment.

3) Since the passive suppression relying on path-loss effect is capable of identically (or in other words, non-selectively) suppressing all the SI components (i.e. including the linear/non-linear components as well as transmitter noise), the analog/digital cancellation techniques should be combined with passive suppression techniques for sufficiently attenuating the SI power as much as possible while without violating the device-size constraints.

4) In light of the fact that predominantly multiantenna aided FD techniques will be utilized in wireless systems in the foreseeable future, the increased DoF offered by the antenna arrays opens up a range of new solutions for SI cancellation. Advanced techniques, such as natural isolation and SD suppression can be specifically developed for FD based MIMO devices in order to facilitate high-performance SI suppression. However, complex matrix computations are required by many of the existing SD suppression methods, such as those involved in pre-coding/decoding and MMSE filtering, etc. This complexity-burden significantly hampers the realizability of FD systems. Hence more cost-efficient algorithms have to be designed by relying on sophisticated yet low-complexity signal processing techniques, especially for highdimensional MIMO channels, in order to facilitate the practical implementation of FD based systems.

5) Apart from the above-mentioned physical-layer solutions, FD research efforts have to be dedicated to the design of cost-effective MAC-layer protocols for multi-node networks. Some of the most challenging problems met in wireless networks, such as the problems imposed by hidden terminals, the throughput erosion due to network congestion and their high end-to-end delay may be mitigated by efficient FD MAC-layer protocols. However, a number of open challenges encountered during the design of FD MAC protocols have to be tackled. On one hand, the FD based MAC-layer protocols must be backwards compatible with the existing HD MAC protocols. Specifically, a practically implemented FD system must be capable of providing a fair opportunity for all HD/FD nodes to access the shared medium, whilst equipping the FD devices with the capability of identifying the duplex mode of the other devices. On the other hand, the efficiency degradation encountered in handling asymmetric traffic must be mitigated in FD MAC-layer protocols, otherwise the FD gains potentially offered by physical-layer techniques may be eroded by an inefficient MAClayer protocol. Furthermore, in light of the fact that the FD mode may not always be capable of outperforming its HD counterpart, hybrid schemes switching between the HD and FD modes should be supported by the FD protocols for adaptively exploiting the radio resources and hence for maximizing the attainable spectral efficiency.

6) Although the existing studies showed that the FD mode is capable of providing a promising performance gain over its HD counterpart in terms of its physical-layer throughput in bidirectional communication scenarios, most existing studies evaluate the FD gains by considering the simple idealized communication scenarios of bidirectional links supporting symmetric traffic [10], without investigating the FD gains, when handling the nonideal scenarios of asymmetric traffic. For example, it was shown in [10] that the balun-based FD system provides a higher capacity than the conventional HD mode for a $(2 \times 2)$ MIMO link in its high-SNR regime (e.g. higher than $12 \mathrm{~dB}$ ), provided that the SI-induced SNR loss remains below $1.5 \mathrm{~dB}$. However, without invoking the FD protocols in a more realistic real-life environment subjected to much more severe interference and to realistic traffic characteristics, the attainable FD gains across the whole network require further investigations.

\section{B. Future Research}

Apart from the above-mentioned challenges, it is also worth pointing out that the approaches presented in this paper may be further developed, as detailed below.

1) Although the existing FD techniques are capable of offering an SI cancellation gain that makes the ultimate SINR high enough for high-integrity detection at the receiver, the current analog cancellation circuits are still too bulky on the order of 100 
square centimeters [11]. Thus hampers their employment in cell phones or other portable devices. To operate the SI cancellation modules on such small devices, further miniaturization of the core circuits is required. For example, as speculated in [11], the delay-line circuit could be realized in a sufficiently small radio frequency integrated circuit (RFIC) as compact as 20-30 square millimeters for estimating the true time delays.

2) Despite their substantial research-advances, the current FD designs still under-utilize the spatial diversity order potential offered by multiple antennas, because the state-of-the-art antennas are usually partitioned into transmit and receive subsets in order to enable simultaneous transmission and reception on a single FD mode terminal. A new FD architecture allowing each antenna to support simultaneous transmission and reception on the same channel is thus highly desirable, otherwise the attainable FD gains will be essentially eliminated by the loss of spatial diversity gain. To achieve the above-mentioned objectives, the SI cancellation circuit must be capable of cancelling both the SI induced by each antenna's own transmissions and that imposed by crosstalk amongst the different antennas [11]. Naturally, designing such a powerful, yet compact circuit will impose new challenges, because the SI cancellation will become a multi-parameter-resolution problem, forcing both the hardware complexity as well as the circuit size to increase significantly, as the number of interference sources increases.

3) Sophisticated SI leakage-channel estimators have to be implemented at the FD mode receiver for successfully extracting the CSI of interest from its SI-contaminated counterpart, followed by CSI feedback to the transmitter [10]. However, the existing periodic-sounding based CSI-estimations mechanisms may fail to track the CSI changes in dynamic environment, although they might be adequate for networks experimenting relatively static channel conditions. Specifically, in CRNs the FD radio environment becomes even more challenging than that of HD transmissions, because FD devices suffer from a heavier interference imposed by the secondary nodes than in the HD mode. Therefore, designing robust algorithms relying on much more dynamic CSI estimators for adaptively tracking the channel states (e.g. on a per-packet basis [10]) and for mitigating the SI/noise power in a dynamic manner is critical for FD communications invoked in CR environments.

4) The attainable performance gains of the FD mode may be impacted by several issues, including the SI power, the system's traffic load and the symmetrical characteristics of the traffic streams. For instance, the FD mode outperforms the conventional HD mode of MIMO systems in terms of their throughput, if communications is bidirectional, while the latter performs better if all communications are in one direction [10]. A hybrid scheme facilitating dynamic switching between the HD and FD modes can be implemented to combine both of its constituent modes. However, the hybrid nodes have to be capable of identifying the environment changes in terms of the CSI, the traffic characteristics as well as communication directions and of promptly switching between the FD and HD modes. Furthermore, in the absence of a centralized controller, a sophisticated distributed approach relying on self-organization [56], [121], [122] should also be employed by each node for implementing a cost-efficient hybrid protocol.

5) The power efficiency of the FD mode must be significantly improved either with the aid of circuit architecture optimization or by relying on the enhancement of MAC/higher-layer protocols. As compared to the HD mode, which employs only half of the total resources (i.e. time resources in Time Division Duplexing (TDD) or frequency resources in Frequency Division Duplexing (FDD)) for signal transmissions, a higher power consumption is encountered by the FD devices, which simultaneously perform both transmission and reception by exploiting all the spectral resources, if a sufficiently high tele-traffic load is assumed. Furthermore, the SI-cancellation circuits of the FD devices employed for eliminating the linear/nonlinear SI components from the static/changing environment also impose an extra energy consumption over that of the HD systems. Therefore, systematically solving the high-power-consumption problems encountered in FD networks relying on sophisticated techniques ranging from the circuit level to the protocol level would facilitate powerefficient FD solutions.

6) Numerous critical issues, such as the problems of hidden terminals and multiple access collisions cannot be readily addressed in distributed networks in the context of FD based MAC protocol design. Specifically, the design of an automatic resource allocation technique capable of adapting 
to time-variant traffic load conditions as well as to diverse QoS requirements in non-infrastructurebased networks constitutes a new challenge. Unlike infrastructure-based networks, the family of non-infrastructure-based networks usually perform their networking functions in a self-organized manner without relying on centralized control [56], [121]. Thus an appropriate MAC protocol conceived for fully exploiting the FD benefits whilst successfully addressing a range of challenging issues such as the adaptivity, both to diverse QoS requirements and to complex interference environments, whilst simultaneously maintaining backward-compatibility with the existing MAC protocols in non-infrastructure-based networks is definitely worthy of further study.

7) FD based small cell BSs may provide a promising way towards addressing the complicated backhaul problems encountered in heterogeneous networks, in which the typical backhaul solutions relying on out-of-band resources (e.g. fiber, microwave radio, etc.) become challenging either due to the costprohibitive fiber connecting or due to the LOS requirement of microwave based backhaul [20]. To leverage the same spectrum for both user access and backhaul, the FD based small-cell BSs can receive from the macrocells while simultaneously transmit to the UEs in the downlink. In the uplink, on the other hand, the FD based small-cell BSs will receive from the UEs while simultaneously transmit to the macrocells. In this manner, the FD based small-cell BSs can effectively backhaul itself without requiring a separate backhaul frequency band, thus substantially reducing the cost and complexity of implementing heterogeneous networks. The existed studies show [20] that the self-backhauled small cells may even perform nearly as well as a fiber-backhauled small cells. Furthermore, by virtue of the larger form factor, the self-backhauled small-cell BSs may achieve much higher spectral efficiencies than the size-constrained FD devices. However, to provide an improved FD gain in the self-backhauled BS with a larger form factor, a higher ordered MIMO comprising much larger directional backhaul antennas are usually required, thus substantially increasing the burden of FD receiver from the perspective of complicated SI cancellation as well as the other signal processing functionalities. Therefore, proposing a feasible FD based self-backhaul solution enabling a more efficient utilization of the macrocell spectral re- sources, whilst simultaneously facilitating a costefficient high-ordered MIMO based SI cancellation constitutes a promising study-item.

Given that FD communication has become a feasible design option, the research community is turning its attention to more cost-effective system design principles, where the attainable spectral efficiency may be significantly improved by creating high-reliability, reducedcomplexity, reduced-cost, power-efficient FD devices. We expect a substantial throughput increase upon simultaneously enabling the reliable transmission and reception of data by a single device, as facilitated by highperformance SI cancellation, adaptive power allocation, and hybrid HD/FD schemes combined with a CR-aided networking. In a nutshell, an exciting era for a growing research-community! 


\section{NOMENCLATURE}

\begin{tabular}{|c|c|}
\hline $\mathrm{ADC}$ & analog-to-digital converter \\
\hline $\mathrm{AF}$ & amplify-and-forward \\
\hline AGC & automatic gain control \\
\hline AP & access point \\
\hline AS & antenna separation \\
\hline $\begin{array}{l}\text { ASAC } \\
\text { ation }\end{array}$ & Antenna Separation and Analog Cancel- \\
\hline $\begin{array}{c}\text { ASADC } \\
\text { Cancellation }\end{array}$ & Antenna Separation, Analog and Digital \\
\hline $\begin{array}{l}\text { ASDC } \\
\text { ion }\end{array}$ & Antenna Separation and Digital Cancella- \\
\hline BER & Bit Error Ratio \\
\hline BPSK & binary phase shift keying \\
\hline BS & base station \\
\hline CR & cognitive radio \\
\hline CRN & cognitive radio network \\
\hline CSI & channel state information \\
\hline CSMA & Carrier Sense Multiple Access \\
\hline DF & decode-and-forward \\
\hline DFT & discrete Fourier transform \\
\hline DL & downlink \\
\hline DoF & Degrees of Freedom \\
\hline EPA & Equal Power Allocation \\
\hline FD & full-duplex \\
\hline FDD & Frequency Division Duplexing \\
\hline FHDR & full- and half-duplex relaying \\
\hline HD & half-duplex \\
\hline $\mathrm{HOL}$ & head of line \\
\hline IPC & individual power constrains \\
\hline ISM & Industrial Scientific Medical \\
\hline LOS & line-of-sight \\
\hline MAC & Media Access Control \\
\hline MIMO & Multi-Input Multi-Output \\
\hline MMSE & minimum mean square error \\
\hline MRC & maximum ratio combining \\
\hline NAV & network allocation vectors \\
\hline $\mathrm{NC}$ & network coding \\
\hline NLOS & non-line-of-sight \\
\hline OFDM & orthogonal frequency division multiplexing \\
\hline OFDMA & orthogonal frequency division multiple \\
\hline access & \\
\hline $\mathrm{OP}$ & outage probability \\
\hline OPA & optimal power allocation \\
\hline PLIR & path-loss-to-interference ratio \\
\hline PLR & Packet Loss Ratio \\
\hline QAM & Quadrature Amplitude Modulation \\
\hline QCNTA & Quellan Canceller with Nulling Transmit \\
\hline Antenna & \\
\hline QoS & quality of service \\
\hline RA & Receive Antenna \\
\hline
\end{tabular}

$\begin{array}{ll}\text { RF } & \text { Radio Frequency } \\ \text { RFIC } & \text { radio frequency integrated circuit } \\ \text { RSSI } & \text { Received Signal Strength Indicator } \\ \text { SCSI } & \text { Scaled Cancelling Signal Injection } \\ \text { SD } & \text { spatial-domain } \\ \text { SFN } & \text { single frequency network } \\ \text { SI } & \text { self-interference } \\ \text { SIC } & \text { successive interference cancellation } \\ \text { SIR } & \text { Signal-to-Interference Ratio } \\ \text { SISO } & \text { Single-Input Single-Output } \\ \text { SINR } & \text { Signal-to-Interference-and-Noise Radio } \\ \text { SNR } & \text { Signal-to-Noise Ratio } \\ \text { SPC } & \text { sum power constrains } \\ \text { SRB } & \text { Shared Random Backoff } \\ \text { SVD } & \text { singular value decomposition } \\ \text { TA } & \text { Transmit Antenna } \\ \text { TAS } & \text { Transmit Antenna Selection } \\ \text { TD } & \text { time-domain } \\ \text { TDD } & \text { Time Division Duplexing } \\ \text { TDTB } & \text { time-domain transmit beamforming } \\ \text { WiFi } & \text { Wireless Fidelity } \\ \text { WLAN } & \text { wireless local area networks } \\ \text { ZF } & \text { Zero Forcing } \\ & \end{array}$

\section{REFERENCES}

[1] A. J. Paulraj, D. A. GORE, R. U. Nabar, and H. Bolcskei, "An overview of MIMO communications - a key to gigabit wireless," Proc. IEEE, vol. 92, no. 2, pp. 198-218, July 2004.

[2] Z. Zhang, X. Wang, K. Long, A. V. Vasilakos, and L. Hanzo, "Large-Scale MIMO Based Wireless Backhaul in 5G Networks," IEEE Wireless Commun. Mag., Oct. 2015.

[3] R. van Nee and R. Prasad, OFDM for Wireless Multimedia Communications. Artech House, 2000.

[4] M. Duarte, Full-duplex wireless: Design, implementation and characterization. PhD Dissertation, Rice University, 2012.

[5] Z. Zhang, W. Zhang, and C. Tellambura, "Cooperative OFDM channel estimation in the presence of frequency offsets," IEEE Trans. Veh. Technol., vol. 58, no. 7, pp. 3447-3459, Sept. 2009.

[6] Z. Zhang, X. Chai, K. Long, A. V. Vasilakos, and L. Hanzo, "Full Duplex Techniques for 5G Networks: Self-Interference Cancellation, Protocol Design, and Relay Selection," IEEE Commun. Mag., vol. 53, no. 5, pp. 128-137, May 2015.

[7] J. I. Choi, M. Jain, K. Srinivasan, P. Levis, and S. Katti, "Achieving single channe, full duplex wireless communication," in Proceedings of ACM MobiCom'10, Chicago, Illinois, USA, 2010.

[8] M. Duarte, C. Dick, and A. Sabharwal, "Experiment-Driven Characterization of Full-Duplex Wireless Systems," IEEE Trans. Wireless Commun., vol. 11, no. 12, pp. 4296-4307, Dec. 2012.

[9] H. Ju, E. Oh, and D. Hong, "Improving efficiency of resource usage in two-hop full duplex relay systems based on resource sharing and interference cancellation," IEEE Trans. Wireless Commun., vol. 8, no. 8, pp. 3933-3938, Aug. 2009.

[10] M. Jain, J. I. Choi, T. Kim, D. Bharadia, S. Seth, K. Srinivasan, P. Levis, S. Katti, and P. Sinha, "Practical, real-time, full duplex wireless," in Proceedings of ACM MobiCom'11, Sept. 2011, pp. 301-312.

[11] D. Bharadia, E. Mcmilin, and S. Katti, "Full duplex radios," in Proc. ACM SIGCOMM, Hong Kong, 2013, pp. 375-386. 
[12] M. Duarte and A. Sabharwal, "Full-duplex wireless communications using off-the-shelf radios: Feasibility and first results," in Proc. 2010 Asilomar Conference on Signals and Systems, 2010.

[13] E. Everett, M. Duarte, C. Dick, and A. Sabharwal, "Empowering full-duplex wireless communication by exploiting directional diversity," in Asilomar Conference on Signals, Systems and Computers, 2011, pp. 2002-2006.

[14] B. Radunovic, D. Gunawardena, P. Key, A. Proutiere, N. Singh, V. Balan, and G. Dejean, "Rethinking Indoor Wireless Mesh Design: Low Power, Low Frequency, Full-Duplex," in Wireless Mesh Networks (WIMESH 2010), 2010 Fifth IEEE Workshop on, 2010, pp. 1-6.

[15] A. Sahai, G. Patel, and A. Sabharwal, "Pushing the limits of full-duplex: Design and real-time implementation," [Online]. Available: arXiv:1107.0607, 2011.

[16] Y. Hua, P. Liang, Y. Ma, A. C. Cirik, and Q. Gao, "A method for broadband full-duplex MIMO radio," IEEE Signal Processing Lett., vol. 19, no. 12, pp. 793-796, Dec. 2012.

[17] S. Gollakota and D. Katabi, "ZigZag decoding: combating hidden terminals in wireless networks," in Proc. ACM SIGCOMM, New York, NY, USA, 2008, pp. 159-170.

[18] M. Chung, M. S. Sim, J. Kim, D. K. Kim, and C.-B. Chae, "Prototyping Real-Time Full Duplex Radios," 2015. [Online]. Available: http://arxiv.org/abs/1503.03013v2

[19] T. Riihonen, S. Werner, and R. Wichman, "Mitigation of loopback self-interference in full-duplex MIMO relays," IEEE Trans. Signal Processing, vol. 59, no. 12, pp. 5983-5993, Dec. 2011.

[20] S. Hong, J. Brand, J. I. Choi, M. Jain, J. Mehlman, S. Katti, and P. Levis, "Applications of Self-Interference Cancellation in 5G and Beyond," IEEE Commun. Mag., vol. 52, no. 2, pp. 114-121, Feb. 2014.

[21] Y. Sung, J. Ahn, B. V. Nguyen, and K. Kim, "Loop-interference suppression strategies using antenna selection in full-duplex MIMO relays," in Intelligent Signal Processing and Communications Systems (ISPACS), 2011 International Symposium on, 2011, pp. 1-4.

[22] T. Riihonen, S. Werner, and R. Wichman, "Hybrid FullDuplex/Half-Duplex Relaying with Transmit Power Adaptation," IEEE Trans. Wireless Commun., vol. 10, no. 9, pp. 30743085, Sept. 2011.

[23] 3GPP, "Full duplex configuration of Un and Uu subframes for type I relay," 3GPP TSG RAN WG1 R1-100139, Tech. Rep., Jan. 2010.

[24] — , "Text proposal on inband full duplex relay for TR 36.814," 3GPP TSG RAN WG1 R1-101659, Tech. Rep., Feb. 2010.

[25] B. Radunovic, D. Gunawardena, P. Key, A. Proutiere, N. Singh, H. V. Balan, and G. Dejean, "Rethinking indoor wireless: Low power, low frequency, full-duplex," in Technical Report MSRTR-2009-148, Microsoft Research, 2009.

[26] W. Cheng, X. Zhang, and H. Zhang, "Full/half duplex based resource allocations for statistical quality of service provisioning in wireless relay networks," in INFOCOM, 2012 Proceedings IEEE, Mar. 2012, pp. 864-872.

[27] T. Riihonen, S. Werner, and R. Wichman, "Comparison of full-duplex and half-duplex modes with a fixed amplify-andforward relay," in IEEE Wireless Commun. and Networking Conf. (WCNC), Budapest, Hungary, Apr. 2009, pp. 1-5.

[28] B. Chun and Y. H. Lee, "A spatial self-interference nullification method for full duplex amplify-and-forward MIMO relays," in IEEE Wireless Commun. and Networking Conf. (WCNC), Apr. 2010, pp. 1-6.

[29] A. Sahai, G. Patel, C. Dick, and A. Sabharwal, "On the Impact of Phase Noise on Active Cancelation in Wireless Full-Duplex," IEEE Trans. Veh. Technol., vol. 62, no. 9, pp. 4494-4510, Nov. 2013.
[30] T. Riihonen, S. Werner, R. Wichman, and J. Hamalainen, "Outage probabilities in infrastructure-based single-frequency relay links," in IEEE Wireless Commun. and Networking Conf. (WCNC), Budapest, Hungary, Apr. 2009.

[31] T. Kwon, Y. Lim, and D. Hong, "Comparison of FDR and HDR Under Adaptive Modulation With Finite-Length Queues," IEEE Trans. Veh. Technol., vol. 61, no. 2, pp. 838-843, Feb. 2012.

[32] R. Schoenen, A. Otyakmaz, and B. H. Walke, "Concurrent operation of half-and full-duplex terminals in future multihop FDD based cellular networks," in Int. Conf. on Wireless Commun., Networking and Mobile Computing (WiCom), Oct. 2008, pp. 1-7.

[33] D. Michalopoulos, J. Schlenker, J. Cheng, and R. Schober, "Error rate analysis of full-duplex relaying," in Waveform Diversity and Design Conference (WDD), 2010 International, Aug. 2010, pp. $165-168$.

[34] G. Kramer, M. Gastpar, and P. Gupta, "Cooperative Strategies and Capacity Theorems for Relay Networks," IEEE Trans. Inform. Theory, vol. 51, no. 9, pp. 3037-3063, Sept. 2005.

[35] R. Nikjah and N. C. Beaulieu, "Achievable Rates and Fairness in Rateless Coded Decode-and-Forward Half-Duplex and FullDuplex Opportunistic Relaying," in Proc. IEEE Int. Conf. Communications (ICC), May 2008, pp. 3701-3707.

[36] T. Riihonen, S. Werner, R. Wichman, and E. Z. B., "On the feasibility of full-duplex relaying in the presence of loop interference," in Proc. 10th IEEE Workshop on Signal Processing Advances in Wireless Communications, June 2009.

[37] D. Skraparlis, V. K. Sakarellos, A. D. Panagopoulos, and J. D. Kanellopoulos, "Outage performance analysis of cooperative diversity with MRC and SC in correlated lognormal channels," EURASIP J. Wireless Commun. Networking, vol. 2009, pp. 1-7, 2009.

[38] T. Riihonen, S. Werner, and R. Wichman, "Rate-interference trade-off between duplex modes in decode-and-forward relaying," in IEEE Int. Symposium on Personal, Indoor and Mobile Radio Commun. (PIMRC), Sept. 2010.

[39] T. Kwon, S. Lim, S. Choi, and D. Hong, "Optimal duplex mode for DF relay in terms of the outage probability," IEEE Trans. Veh. Technol., vol. 59, no. 7, pp. 3628-3634, Sept. 2010.

[40] T. Riihonen, S. Werner, and R. Wichman, "Transmit power optimization for multiantenna decode-and-forward relays with loopback self-interference from full-duplex operation," in Signals, Systems and Computers (ASILOMAR), 2011 Conference Record of the Forty Fifth Asilomar Conference on, Nov. 2011, pp. $1408-1412$.

[41] P. T. Hiep and R. Kohno, "Capacity bound for full-duplex multiple-hop MIMO relays system in rayleigh fading," in Wireless Telecommunications Symposium (WTS), 2012, Apr. 2012, pp. 1-6.

[42] H. Alves, G. Fraidenraich, R. D. Souza, M. Bennis, and M. Latva-aho, "Performance Analysis of Full Duplex and Selective and Incremental Half Duplex Relaying Schemes," in IEEE Wireless Commun. and Networking Conf. (WCNC), 2012, pp. 771-775.

[43] Y. R. Zheng and C. Xiao, "Performance of Block-Markov Full Duplex Relaying with Self Interference in Nakagami-m Fading," IEEE Wireless Commun. Lett., vol. 2, no. 3, pp. 311-314, June 2013.

[44] M. Khafagy, A. Ismail, M.-S. Alouini, and S. Aissa, "On the Outage Performance of Full-Duplex Selective Decode-andForward Relaying," IEEE Commun. Lett., vol. 17, no. 6, pp. 1180-1183, 2013.

[45] N. Zlatanov and R. Schober, "Buffer-Aided Half-Duplex Relaying Can Outperform Ideal Full-Duplex Relaying," IEEE Commun. Lett., vol. 17, no. 3, pp. 479-482, Mar. 2013.

[46] B. P. Day, A. R. Margetts, D. W. Bliss, and P. Schniter, "Full-Duplex MIMO Relaying: Achievable Rates Under Limited 
Dynamic Range," IEEE J. Select. Areas Commun., vol. 30, no. 8, pp. 1541-1553, Sept. 2012.

[47] P. Cheng, G. Yu, Z. Zhang, H. Jia, H. H. Chen, and P. Qiu, "Cross-layer performance analysis of two-hop wireless links with adaptive modulation," in Proc. IEEE Int. Symp. Pers., Indoor, Mobile Radio Commun., Sept. 2006, pp. 1-5.

[48] A. Müller and H. C. Yang, "Dual-hop adaptive packet transmission with regenerative relaying for wireless TDD systems," in IEEE Global Telecommn. Conf. (GLOBECOM), Nov. 2009, pp. 552-557.

[49] T. Riihonen, R. Wichman, and J. Hamalainen, "Co-phasing full-duplex relay link with non-ideal feedback information," in Wireless Communication Systems. 2008 (ISWCS'08). IEEE International Symposium on, Oct. 2008, pp. 263-267.

[50] J. Cioffi, G. Dudevoir, M. V. Eyuboglu, and G. J. Forney, "Bypassing orthogonal relaying transmissions via spatial signal separation," IEEE Trans. Commun., vol. 43, no. 10, pp. 2582 2594, Oct. 1995.

[51] T. K. Baranwal, D. S. Michalopoulos, and R. Schober, "Outage Analysis of Multihop Full Duplex Relaying," IEEE Commun. Lett., vol. 17, no. 1, pp. 63-66, Jan. 2013.

[52] P. K. Sharma and P. Garg, "Outage analysis of full duplex decode and forward relaying over Nakagami-m channels," in Communications (NCC), 2013 National Conference on, Feb. 2013, pp. 1-5.

[53] K. Lee, H. M. Kwon, M. Jo, H. Park, and Y. H. Lee, "MMSEBased Optimal Design of Full-Duplex Relay System," in Proc. IEEE Vehicular Technology Conf. (VTC), Sept. 2012, pp. 1-5.

[54] J. Joung and A. Sayed, "Design of half- and full-duplex relay systems based on the MMSE formulation," in Proc. of IEEE/SP SSP'09, Sept. 2009, pp. 281-284.

[55] B. Chun, E. Jeong, J. Joung, Y. Oh, and Y. H. Lee, "Pre-nulling for self-interference suppression in full-duplex relays," in Proc Asia-Pacific Signal and Information Processing Association Annual Summit Conference (APSIPA ASC), Sapporo, Oct. 2009.

[56] Z. Zhang, K. Long, and J. Wang, "Self-Organization Paradigms and Optimization Approaches for Cognitive Radio Technologies: A Survey," IEEE Wireless Commun. Mag., vol. 20, no. 2, pp. 36-42, Apr. 2013.

[57] J. W. Tantra, C. H. Foh, and D. Qiu, "On link reliability in wireless mobile ad hoc networks," in Vehicular Technology Conference, 2006. VTC-2006 Fall. 2006 IEEE 64th, 2006, pp. $1-5$.

[58] T. Lee, The Design of CMOS Radio-Frequency Integrated Circuits. Cambridge University Press, 2004.

[59] M. E. Knox, "Single antenna full duplex communications using a common carrier," in Wireless and Microwave Technology Conference (WAMICON), 2012 IEEE 13th Annual. IEEE, 2012, pp. 1-6.

[60] E. Everett, Full-duplex Infrastructure Nodes: Achieving Long Range with Half-duplex Mobiles. Master's thesis, Rice University, 2012.

[61] C. R. Anderson, S. Krishnamoorthy, C. G. Ranson, T. J. Lemon, W. G. Newhall, T. Kummetz, and J. H. Reed, "Antenna isolation, wideband multipath propagation measurements, and interference mitigation for on-frequency repeaters," in Proc. SoutheastCon, Greensboro, NC, Mar. 2004, pp. 110-114.

[62] D. Bliss, P. Parker, and A. Margetts, "Simultaneous transmission and reception for improved wireless network performance," in Proc IEEE Statistical Signal Processing, 2007, pp. 478-482.

[63] K. Haneda, E. Kahra, S. Wyne, C. Icheln, and P. Vainikainen, "Measurement of loop-back interference channels for outdoorto-indoor full-duplex radio relays," in Proc. The 4th European Conf Ant. Prop., Apr. 2010, pp. 1-5.

[64] A. Khandani, "Shaping the Future of Wireless: TwoWay Connectivity." [Online]. Available: http://www.nortelinstitute.uwaterloo.ca/content/Shaping Future of Wireless Twoway Connectivity_18June2012.pdf
[65] D. Halperin, T. Anderson, and D. Wetherall, "Taking the sting out of carrier sense: interference cancellation for wireless lans," in Proceedings of the 14th ACM international conference on Mobile computing and networking (MobiCom'08), New York, NY, USA, 2008, pp. 339-350.

[66] M. A. A. Khojastepour and S. Rangarajan, "Wideband Digital Cancellation for Full-Duplex Communications," in Signals, Systems and Computers (ASILOMAR), 2012 Conference Record of the Forty Sixth Asilomar Conference on, Nov. 2012, pp. 13001304.

[67] H. Ju, S. Lee, K. Kwak, E. Oh, and D. Hong, "A new duplex without loss of data rate and utilizing selection diversity," in Proc. IEEE Vehicular Technology Conf. (VTC), May 2008, pp. 1519-1523.

[68] T. Riihonen, S. Werner, and R. Wichman, "Spatial loop interference suppression in full-duplex MIMO relays," in Proc. 43rd Asilomar Conf. Signals, Systems, Computers (ACSSC'09), Pacific Grove, CA, Nov. 2009.

[69] H. Ju, E. Oh, and D. Hong, "Catching resource-devouring worms in next-generation wireless relay systems: Two-way relay and full-duplex relay," IEEE Commun. Mag., vol. 47, no. 9, pp. 58-65, Sept. 2009.

[70] J.-H. Lee and O.-S. Shin, "Full-duplex relay based on block diagonalisation in multiple-input multiple-output relay systems," IET Commun., vol. 4, no. 15, pp. 1817-1826, 2010.

[71] E. Everett, D. Dash, C. Dick, and A. Sabharwal, "Selfinterference cancellation in multi-hop full-duplex networks via structured signaling," in Allerton Conference on Commun., Control, and Comput., 2011, pp. 1619-1626.

[72] T. Riihonen, A. Balakrishnan, K. Haneda, S. Wyne, S. Werner, and R. Wichman, "Optimal eigenbeamforming for suppressing self-interference in full-duplex MIMO relays," in Information Sciences and Systems (CISS), 2011 45th Annual Conference on, 2011, pp. 1-6.

[73] R. Lopez-Valcarce, E. Antonio-Rodriguez, C. Mosquera, and F. Perez-Gonzalez, "An Adaptive Feedback Canceller for FullDuplex Relays Based on Spectrum Shaping," IEEE J. Select. Areas Commun., vol. 30, no. 8, pp. 1566-1577, Sept. 2012.

[74] B. Chun and H. Park, "A Spatial-Domain Joint-Nulling Method of Self-Interference in Full-Duplex Relays," IEEE Commun. Lett., vol. 16, no. 4, pp. 436-438, Apr. 2012.

[75] V. Stanković and P. Spalević, "Cooperative relaying with block DFT processing and full-duplex relays," IEE Elect. Lett., vol. 49, no. 4, pp. 300-302, Feb. 2013.

[76] S. Li and R. D. Murch, "An Investigation Into Baseband Techniques for Single-Channel Full-Duplex Wireless Communication Systems," IEEE Trans. Wireless Commun., vol. 13, no. 9, pp. 4794-4806, Sept. 2014.

[77] A. V. Oppenheim, R. W. Schafer, and J. R. Buck, Discrete-time signal processing (2nd ed.). Prentice-Hall, Inc., Upper Saddle River, NJ, USA, 1999.

[78] Q. Inc., "Qhx220 narrowband noise canceller ic." [Online]. Available: http://www.quellan.com/products/qhx220_ic.php

[79] T. Riihonen and R. Wichman, "Analog and digital selfinterference cancellation in full-duplex MIMO-OFDM transceivers with limited resolution in A/D conversion," in Signals, Systems and Computers (ASILOMAR), 2012 Conference Record of the Forty Sixth Asilomar Conference on, 2012, pp. 45-49.

[80] L. L. Scharf, Statistical Signal Processing: Detection, Estimation, and Time Series Analysis. ADDISON-WESLEY Publishing Company, 1991.

[81] D. Senaratne and C. Tellambura, "Beamforming for space division duplexing," in Proc. IEEE Int. Conf. Communications (ICC), Kyoto, Japan, June 2011, pp. 1-5.

[82] T. Riihonen, S. Werner, and R. Wichman, "Residual selfinterference in full-duplex MIMO relays after null-space projec- 
tion and cancellation," in Proc. IEEE Asilomar Conf. Signals, Syst. Comput., Nov. 2010, pp. 653-657.

[83] A. Thangaraj, R. Ganti, and S. Bhashyam, "Self-interference cancellation models for full-duplex wireless communications," in Signal Processing and Communications (SPCOM), 2012 International Conference on, 2012, pp. 1-5.

[84] D. A. Gore and A. . Paulraj, "MIMO Antenna Subset Selection With Space-Time Coding," IEEE Trans. Signal Processing, vol. 50, no. 10, pp. 2580-2588, Oct. 2002.

[85] A. Hazmi, J. Rinne, and M. Renfors, "Diversity based DVB$\mathrm{T}$ in-door repeater in slowly mobile loop interference environment," in in Proc. 10th Int. OFDM-Workshop, 2005.

[86] T. Taniguchi and Y. Karasawa, "Design and analysis of MIMO multiuser system using full-duplex multiple relay nodes," in Wireless Days (WD), 2012 IFIP, Nov. 2012, pp. 1-8.

[87] I. Krikidis, H. A. Suraweera, S. Yang, and K. Berberidis, "Full-Duplex Relaying over Block Fading Channel: A Diversity Perspective," IEEE Trans. Wireless Commun., vol. 11, no. 12, pp. 4524-4535, Dec. 2012

[88] P. Larsson and M. Prytz, "MIMO On-Frequency Repeater with Self-Interference Cancellation and Mitigation," in Proc. IEEE Vehicular Technology Conf. (VTC), Apr. 2009, pp. 1-5.

[89] J.-H. Lee and O.-S. Shin, "Distributed beamforming approach to full-duplex relay in multiuser MIMO transmission," in IEEE Wireless Commun. and Networking Conf. (WCNC), Apr. 2012, pp. 278-282.

[90] P. Lioliou, M. Viberg, M. Coldrey, and F. Athley, "Selfinterference suppression in full-duplex MIMO relays," in Signals, Systems and Computers (ASILOMAR), 2010 Conference Record of the Forty Fourth Asilomar Conference on, 2010, pp. 658-662.

[91] J. Sangiamwong, T. Asai, J. Hagiwara, Y. Okumura, and T. Ohya, "Joint Multi-Filter Design for Full-Duplex MU-MIMO Relaying," in Proc. IEEE Vehicular Technology Conf. (VTC), Apr. 2009, pp. 1-5.

[92] C.-B. Chae, T. Tang, R. W. Heath, and S. Cho, "MIMO Relaying With Linear Processing for Multiuser Transmission in Fixed Relay Networks," IEEE Trans. Signal Processing, vol. 56, no. 2 , pp. 727-738, Feb. 2008.

[93] S. Li and R. D. Murch, "Full-Duplex Wireless Communication using Transmitter Output Based Echo Cancellation," in IEEE Global Telecommn. Conf. (GLOBECOM), Houston, TX, Dec. 2011, pp. 1-5.

[94] P. Smith, Little known characteristics of phase noise. Analog Devices, Inc., Application Note AN-741., Aug. 2004.

[95] C. Luschi, M. Sandell, P. Strauch, J.-J. Wu, C. Ilas, P.W. Ong, R. Baeriswyl, F. Battaglia, S. Karageorgis, and R.H. Yan, "Advanced signal-processing algorithms for energyefficient wireless communications," Proc. IEEE, vol. 88, no. 10, pp. $1633-1650$, Oct. 2000.

[96] T. U. Rice Univ., Houston, "WARP project." [Online]. Available: warp.rice.edu

[97] D. W. K. Ng and R. Schober, "Dynamic Resource Allocation in OFDMA Systems with Full-Duplex and Hybrid Relaying," in Proc. IEEE Int. Conf. Communications (ICC), Kyoto, Japan, June 2011, pp. 1-6.

[98] M. A. Khojastepour, K. Sundaresan, S. Rangarajan, X. Zhang, and S. Barghi, "The case for antenna cancellation for scalable full duplex wireless communications," in Proc. 2011 ACM Workshop Hot Topics Netw., 2011.

[99] W. Mesbah and T. N. Davidson, "Optimal Power Allocation for Full-Duplex Cooperative Multiple Access," in Proc. IEEE Int. Conf. Acoustics, Speech, and Signal Processing (ICASSP), vol. 4, May 2006, pp. 689-692.

[100] K. Shibuya, "Broadcast-wave relay technology for digital terrestrial television broadcasting," Proc. IEEE, vol. 94, no. 1, pp. 269-273, 2006
[101] T. Riihonen, S. Werner, and R. Wichman, "Optimized gain control for single-frequency relaying with loop interference," IEEE Trans. Wireless Commun., vol. 8, no. 6, pp. 2801-2806, June 2009.

[102] Y.-K. Song and D. Kim, "Convergence of distributed power control with full-duplex amplify-and-forward relays," in Wireless Communications Signal Processing, 2009. WCSP 2009. International Conference on, Nov. 2009, pp. 1-5.

[103] X. Rui, J. Hou, and L. Zhou, "On the performance of full-duplex relaying with relay selection," IEE Elect. Lett., vol. 46, no. 25, pp. 1674-1676, Dec. 2010.

[104] Y. Yang, J. Ge, and Y. Gao, "Power Allocation for Two-Way Opportunistic Amplify-and-Forward Relaying over Nakagamim Channels," IEEE Trans. Wireless Commun., vol. 10, no. 7, pp. 2063-2068, July 2011.

[105] K. Yamamoto, K. Haneda, H. Murata, and S. Yoshida, "Optimal Transmission Scheduling for a Hybrid of Full- and Half-Duplex Relaying," IEEE Commun. Lett., vol. 15, no. 3, pp. 305-307, Mar. 2011.

[106] M. Miyagoshi, K. Yamamoto, K. Haneda, H. Murata, and S. Yoshida, "Multi-user transmission scheduling for a hybrid of full-and half-duplex relaying," in Information, Communications and Signal Processing (ICICS) 2011 8th International Conference on, 2011, pp. 1-5.

[107] I. Krikidis, H. A. Suraweera, P. J. Smith, and C. Yuen, "FullDuplex Relay Selection for Amplify-and-Forward Cooperative Networks," IEEE Trans. Wireless Commun., vol. 11, no. 12, pp. 4381-4393, Dec. 2012.

[108] B. Zhong, D. Zhang, Z. Zhang, Z. Pan, K. Long, and A. V. Vasilakos, "Opportunistic Full-Duplex Relay Selection for Decodeand-Forward Cooperative Networks over Rayleigh Fading Channels," in Proc. IEEE Int. Conf. Communications (ICC), Sydney, Australia, June 2014, pp. 1-6.

[109] J.-H. Lee and O.-S. Shin, "Full-Duplex Relay Based on Distributed Beamforming in Multiuser MIMO Systems," IEEE Trans. Veh. Technol., vol. 62, no. 4, pp. 1855-1860, May 2013.

[110] G. Zheng, I. Krikidis, and B. Ottersten, "Full-Duplex Cooperative Cognitive Radio with Transmit Imperfections," IEEE Trans. Wireless Commun., vol. 12, no. 5, pp. 2498-2511, 2013.

[111] A. Otyakmaz, R. Schoenen, and B. Walke, "Parallel Operation of Halfand Full-Duplex FDD in Future Multi-Hop Mobile Radio Networks," in European Wireless Conference, Prague, June 2008, pp. 1-7.

[112] F. Kelly, "Charging and rate control for elastic traffic," Euro. Trans. Telecommun., vol. 8, pp. 33-37, Jan.-Feb. 1997.

[113] K.-K. Wong, "Maximizing the Sum-Rate and Minimizing the Sum-Power of a Broadcast 2-User 2-Input Multiple-Output Antenna System Using a Generalized Zeroforcing Approach," IEEE Trans. Wireless Commun., vol. 5, no. 12, pp. 3406-3412, Dec. 2006

[114] D. B. da Costa and S. Aissa, "Performance analysis of relay selection techniques with clustered fixed-gain relays," IEEE Signal Processing Lett., vol. 17, pp. 201-204, Feb. 2010.

[115] Z. Zhang, C. Tellambura, and R. Schober, "Improved OFDMA uplink transmission via cooperative relaying in the presence of frequency offsetsłpart I: ergodic information rate analysis," Euro. Trans. Telecommun., vol. 21, no. 3, pp. 224-240, Apr. 2010.

[116] _ - "Improved OFDMA uplink transmission via cooperative relaying in the presence of frequency offsets - part II: Outage information rate analysis," Euro. Trans. Telecommun., vol. 21, no. 3, pp. 241-250, Apr. 2010.

[117] I. Krikidis, H. A. Suraweera, and C. Yuen, "Amplify-andForward with full-duplex relay selection," in Proc. IEEE Int. Conf. Communications (ICC), June 2012, pp. 1-6.

[118] T. Riihonen, S. Werner, and R. Wichman, "Hybrid FullDuplex/Half-Duplex Relaying with Transmit Power Adapta- 
tion," IEEE Trans. Wireless Commun., vol. 10, no. 9, pp. 3074 3085, 2011.

[119] H. Kim, S. Lim, H. Wang, and D. Hong, "Optimal Power Allocation and Outage Analysis for Cognitive Full Duplex Relay Systems," IEEE Trans. Wireless Commun., vol. 11, no. 10, pp. 3754-3765, Oct. 2012.

[120] X. Kang, R. Zhang, Y. C. Liang, and H. K. Garg, "Optimal power allocation strategies for fading cognitive radio channels with primary user outage constraint," IEEE J. Select. Areas Commun., vol. 29, no. 2, pp. 374-383, Feb. 2011.

[121] Z. Zhang, K. Long, J. Wang, and F. Dressler, "On Swarm Intelligence Inspired Self-Organized Networking: Its Bionic Mechanisms, Designing Principles and Optimization Approaches," IEEE Commun. Surv. Tut., vol. 16, no. 1, pp. 513-537, FIRST QUARTER 2014.

[122] Z. Zhang, W. Huangfu, K. Long, X. Zhang, X. Liu, and B. Zhong, "On the Designing Principles and Optimization Approaches of Bio-Inspired Self-Organized Network: A Survey," Science China Information Sciences., vol. 56, no. 7, July 2013.

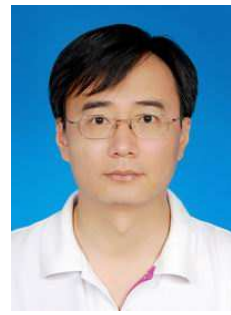

Zhongshan Zhang received the B.E. and M.S. degrees in computer science from the Beijing University of Posts and Telecommunications (BUPT) in 1998 and 2001, respectively, and received Ph.D. degree in electrical engineering in 2004 from BUPT. From Aug. 2004 he joined DoCoMo Beijing Laboratories as an associate researcher, and was promoted to be a researcher in Dec. 2005. From Feb. 2006, he joined University of Alberta, Edmonton, AB, Canada, as a postdoctoral fellow. From Apr. 2009, he joined the Department of Research and Innovation (R\&I), Alcatel-Lucent, Shanghai, as a Research Scientist. From Aug. 2010 to Jul. 2011, he worked in NEC China Laboratories, as a Senior Researcher. He served or is serving as a Guest Editor and/or an editor for several technical journals, such as the IEEE COMMUNICATIONS MAGAZINE and KSII TRANSACTIONS ON INTERNET AND INFORMATION SYSTEMS. He is currently a professor of the School of Computer and Communication Engineering in the University of Science and Technology Beijing (USTB). His main research interests include statistical signal processing, fullduplex communications, Massive MIMO, self-organized networking and cooperative communications.

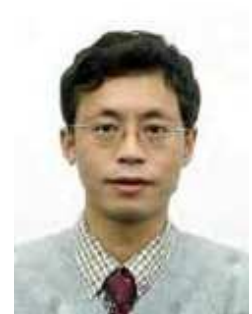

Keping Long received his M.S. and Ph.D. Degrees at UESTC in 1995 and 1998, respectively. From Sep. 1998 to Aug. 2000, he worked as a postdoctoral research fellow at National Laboratory of Switching technology and telecommunication networks in Beijing University of Posts and Telecommunications (BUPT). From Sep. 2000 to Jun. 2001, he worked as an associate professor at Beijing University of Posts and Telecommunications (BUPT). From Jul. 2001 to Nov. 2002, he was a research fellow in ARC Special Research Centre for Ultra Broadband Information Networks (CUBIN) at the University of Melbourne, Australia.

$\mathrm{He}$ is now a professor and dean at School of Computer \& Communication Engineering (CCE), University of Science and Technology Beijing (USTB). He is the IEEE senior member, and the Member of Editorial Committee of Sciences in China Series $\mathrm{F}$ and China Communications. He is also the TPC and the ISC member for COIN2003/04/05/06/07/08/09/10, IEEE IWCN2010, ICON04/06, APOC2004/06/08, Co-chair of organization member for IWCMC2006, TPC chair of COIN2005/2008, TPC Co-chair of COIN2008/2010, He was awarded for the National Science Fund for Distinguished Young Scholars of China in 2007, selected as the Chang Jiang Scholars Program Professor of China in 2008. His research interests are Optical Internet Technology, New Generation Network Technology, Wireless Information Network, Value-added Service and Secure Technology of Network. He has published over 200 papers, 20 keynotes speaks and invited talks in the international conferences and local conferences.

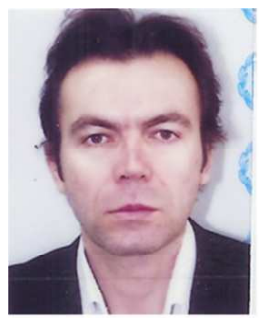

Athanasios V. Vasilakos is currently a Professor at the Lulea University of Technology, Sweden. He has authored or co-authored over 200 technical papers in major international journals and conferences. He is author/coauthor of five books and 20 book chapters in the area of communications. Prof. Vasilakos has served as General Chair/Technical Program Committee Chair for many international conferences. He served or is serving as an Editor and/or Guest Editor for many technical journals, such as the IEEE TRANSACTIONS ON NETWORK AND SERVICE MANAGEMENT, the IEEE TRANSACTIONS ON SYSTEMS, MAN, AND CYBERNETICS, PART B: CYBERNETICS, the IEEE TRANSACTIONS ON INFORMATION TECHNOLOGY IN BIOMEDICINE, the IEEE TRANSACTIONS ON COMPUTERS, the ACM Transactions on Autonomous and Adaptive Systems, IEEE JSAC special issues of May 2009, Jan. 2011, and March 2011, IEEE Communications Magazine, ACM/Springer Wireless Networks (WINET), and ACM/Springer Mobile Networks and Applications (MONET). He is founding Editor-in-Chief of the International Journal of Adaptive and Autonomous Communications Systems (IJAACS) and the International Journal of Arts and Technology (IJART). He is General Chair of the Council of Computing of the European Alliances for Innovation. 


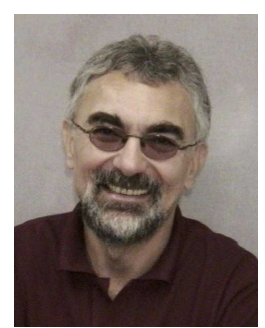

Lajos Hanzo (http://wwwmobile.ecs.soton.ac.uk) FREng, FIEEE FIET, Fellow of EURASIP, DSc received his degree in electronics in 1976 and his doctorate in 1983. In 2009 he was awarded an honorary doctorate by the Technical University of Budapest, while in 2015 by the University of Edinburgh. During his 38-year career in telecommunications he has held various research and academic posts in Hungary, Germany and the UK. Since 1986 he has been with the School of Electronics and Computer Science, University of Southampton, UK, where he holds the chair in telecommunications. He has successfully supervised about 100 $\mathrm{PhD}$ students, co-authored 20 John Wiley/IEEE Press books on mobile radio communications totalling in excess of 10000 pages, published $1500+$ research entries at IEEE Xplore, acted both as TPC and General Chair of IEEE conferences, presented keynote lectures and has been awarded a number of distinctions. Currently he is directing a 60-strong academic research team, working on a range of research projects in the field of wireless multimedia communications sponsored by industry, the Engineering and Physical Sciences Research Council (EPSRC) UK, the European Research Council's Advanced Fellow Grant and the Royal Society's Wolfson Research Merit Award. He is an enthusiastic supporter of industrial and academic liaison and he offers a range of industrial courses. $\mathrm{He}$ is also a Governor of the IEEE VTS. During 2008 - 2012 he was the Editor-in-Chief of the IEEE Press and a Chaired Professor also at Tsinghua University, Beijing. His research is funded by the European Research Council's Senior Research Fellow Grant. For further information on research in progress and associated publications please refer to http://www-mobile.ecs.soton.ac.uk Lajos has 23 000+ citations. 